\title{
WestVirginiaUniversity
}

THE RESEARCH REPOSITORY @ WVU

Graduate Theses, Dissertations, and Problem Reports

2009

\section{Wireless sensor networks in underground structures}

Derrick Morgan

West Virginia University

Follow this and additional works at: https://researchrepository.wvu.edu/etd

\section{Recommended Citation}

Morgan, Derrick, "Wireless sensor networks in underground structures" (2009). Graduate Theses, Dissertations, and Problem Reports. 2076.

https://researchrepository.wvu.edu/etd/2076

This Thesis is protected by copyright and/or related rights. It has been brought to you by the The Research Repository @ WVU with permission from the rights-holder(s). You are free to use this Thesis in any way that is permitted by the copyright and related rights legislation that applies to your use. For other uses you must obtain permission from the rights-holder(s) directly, unless additional rights are indicated by a Creative Commons license in the record and/ or on the work itself. This Thesis has been accepted for inclusion in WVU Graduate Theses, Dissertations, and Problem Reports collection by an authorized administrator of The Research Repository @ WVU. For more information, please contact researchrepository@mail.wvu.edu. 
Wireless Sensor Networks in Underground Structures

\title{
Derrick Morgan
}

\author{
Thesis submitted to the \\ College of Engineering and Mineral Resources \\ at West Virginia University \\ in partial fulfillment of the requirements \\ for the degree of
}

\author{
Master of Science \\ in \\ Mechanical Engineering
}
Larry E. Banta, Ph.D., Chair
Kenneth Means, Ph.D.
Gregory Thompson, Ph.D.

Department of Mechanical Engineering

\author{
Morgantown, West Virginia \\ 2009
}

Keywords: Wireless; Networks; Sensor; WSN

Copyright 2009 Derrick Morgan 


\section{Abstract \\ Wireless Sensor Networks in Underground Structures}

\section{Derrick Morgan}

With the growing interest in developing a system capable of monitoring the health of an underground structure, the research and design of such a system is a logical step. The objective of the research performed was to take that step and develop a system capable of monitoring the health of an underground structure. In order to initiate the process, requirements of the system or network to be developed were set so that the research and design would have some guidance.

The research that followed from the requirements led to the selection of a wireless network which utilizes the Zigbee protocol. Various vendors offering hardware which makes use of the selected Zigbee technology were then researched. Crossbow was selected as the most suitable vendor and components for the network were purchased. Following the acquisition of the hardware, a range test, power consumption test, and a data latency test were performed as design tests. An alarm test, topology test, calibration processes, and a small scale demonstration were also performed, but were treated as system verification tests.

The overall scope of this research was accomplished. A wireless sensor network was successfully designed and validated through testing of the network characteristics and components. 


\section{Acknowledgements}

First and foremost, I would like to offer my deepest thanks to everyone involved in my undergraduate and graduate studies, my research project and thesis, and me achieving my Masters degree.

This thesis would not have been possible without Dr. Larry Banta. Dr. Banta assisted me in pursuing my Masters degree in many ways ranging from offering me a research position with him, to final changes to my thesis and everything in between. Dr. Banta was there to encourage me and challenge me throughout my undergraduate studies as well as graduate studies. Without him this thesis could not have been completed and I would not have earned my Masters degree. I could not be more grateful to have had an advisor/supervisor who is as friendly, kind, and willing to help as Dr. Banta.

I would also like to thank the Professors at West Virginia as a whole. The education that I have received from my undergraduate and graduate studies has proven to be very valuable in my pursuit of my Masters degree, throughout my research, and in starting my career as an engineer. I would like to offer a special thanks to the professors involved with my thesis as committee members; Dr. Kenneth Means, Dr. Greg Thompson, and Dr. Larry Banta.

I am very lucky and thankful to have worked with the research team that I did. I would like to thank Chris Griffin, Jared Grimm, Josh Sill, Javier Martinez, Dr. Kenneth Means, Dr. Wade Huebsch, Dr. Greg Thompson, Dr. Ever Barbero, Dr. Julio Davalos, and again, Dr. Larry Banta. I owe a special thank you to Jamie Wood and Mark Pitts for helping me finalize some of the tests, setting up the necessary network components for the small scale demonstration, and recording the data throughout the demonstration.

I am grateful for the friendly students in the engineering school at WVU. I appreciate their willingness to work with me whether it was in small group assignments or just a general homework questions. I would like to show my gratitude to a specific group of friends and colleagues that had a larger role in my success throughout my studies; Kevin Shields, Ray Tincher, Scott Jones, Mike Ursic, and Wes Hardin.

The College of Engineering and Mineral Resources at WVU provided me with any additional support and equipment that I needed throughout my research, and for this I am very thankful.

I thank my friends and family for supporting me through all of my studies and for encouraging me to pursue my Masters degree. I am indebted greatly to my parents and offer them a special thank you for the amount of support and encouragement they have offered me in my schooling and throughout my life. They have offered financial, emotional, and physical support for many things that helped me earn my degrees and I can never begin to repay them for that. 
Finally, I would like to thank God because I have been very blessed to have all of these people and things to be thankful for. I own him everything, and have become the person I am today and have accomplished everything I have through him. 


\section{Table of Contents}

Abstract ........................................................................... ii

Acknowledgements .....................................................ii

Table of Contents ............................................................ V

List of Tables ................................................................

List of Figures........................................................... viii

Chapter 1: Introduction............................................. 1

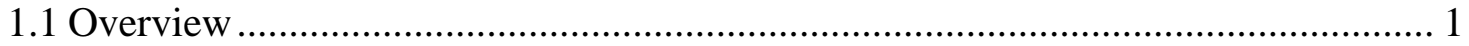

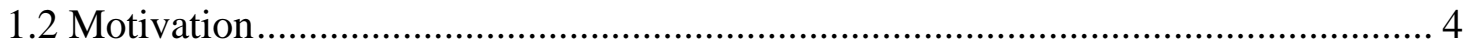

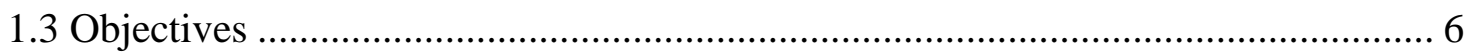

Chapter 2: Background ............................................... 7

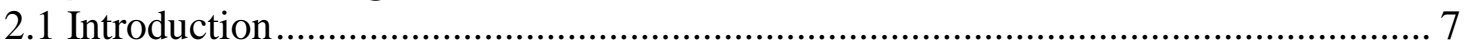

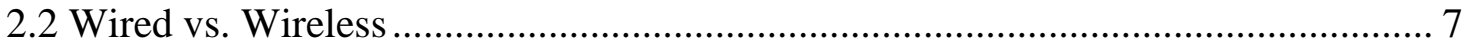

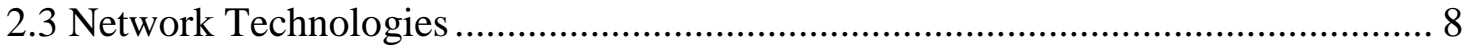

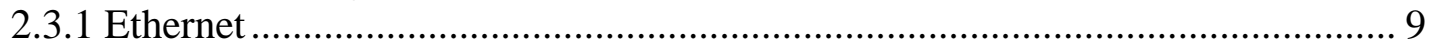

2.3.2 Wireless Networks ........................................................................... 9

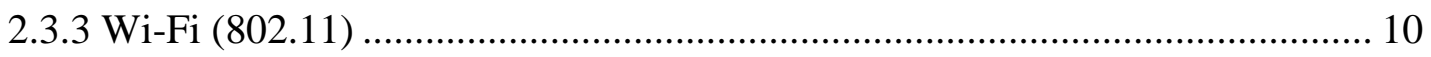

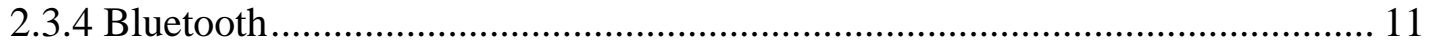

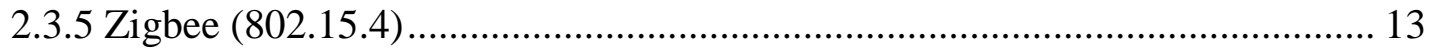

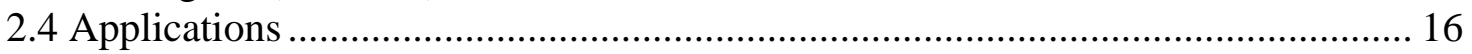

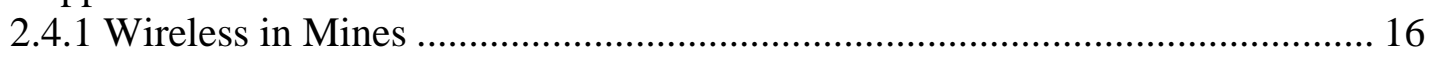

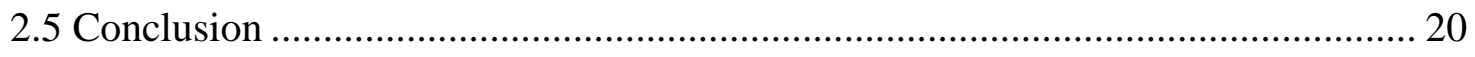

Chapter 3: Technical Approach.....................................21

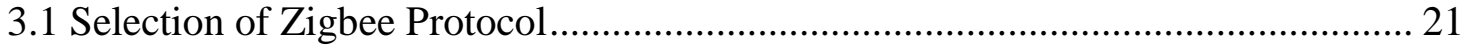

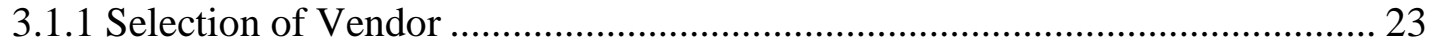

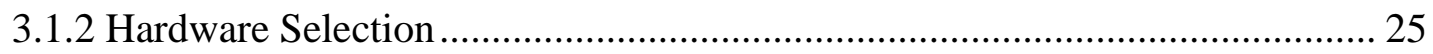

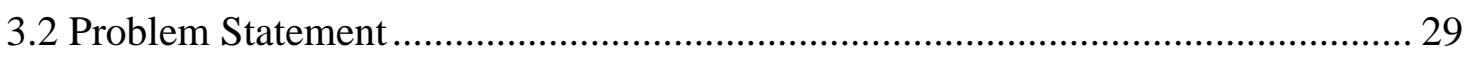

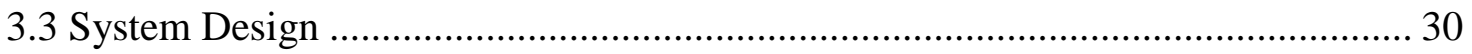

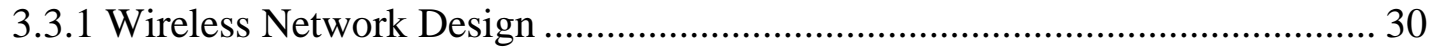

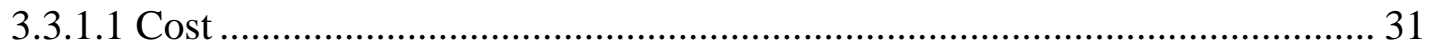

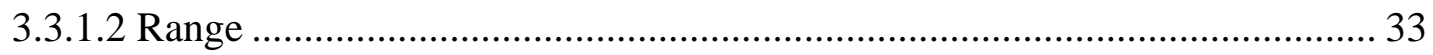

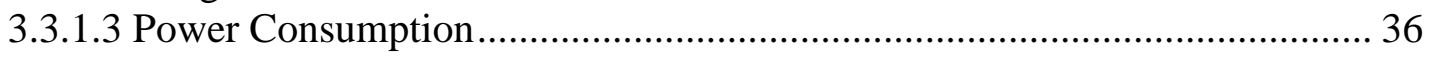

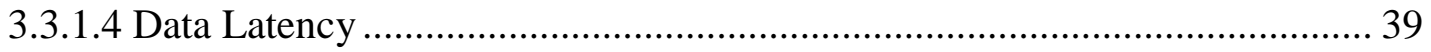

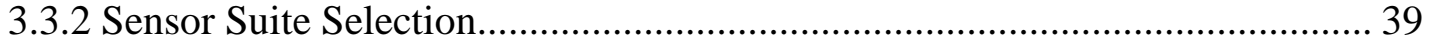

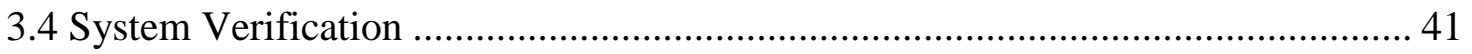

3.4.1 Alarm Testing ................................................................................. 41

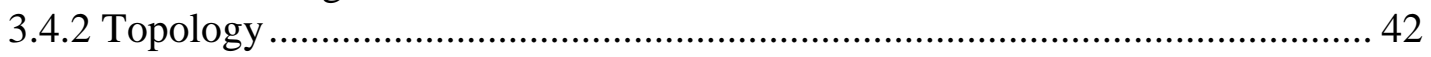

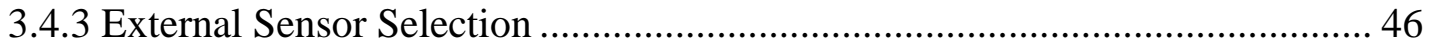

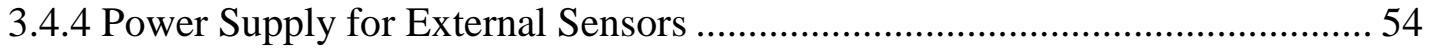

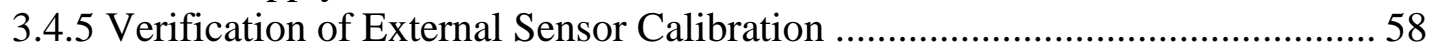

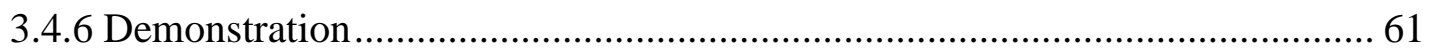

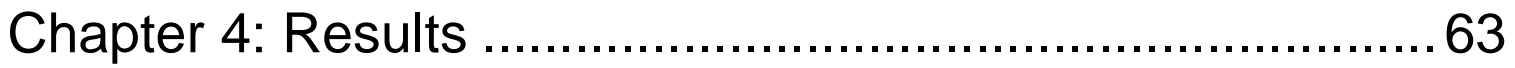




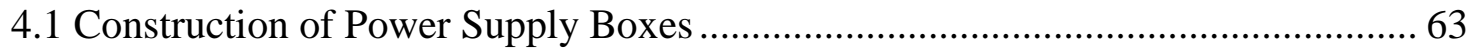

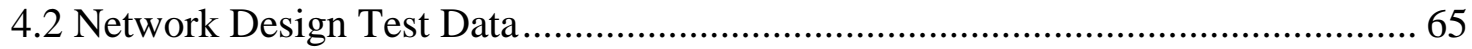

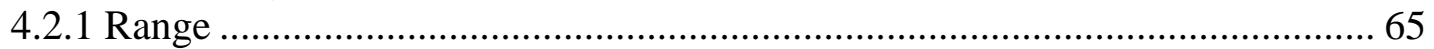

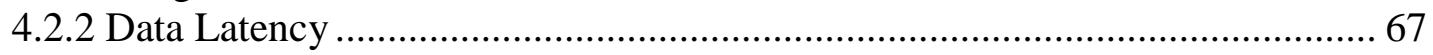

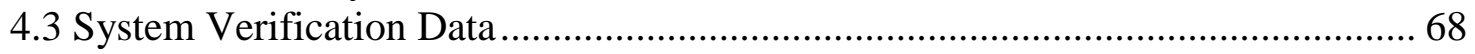

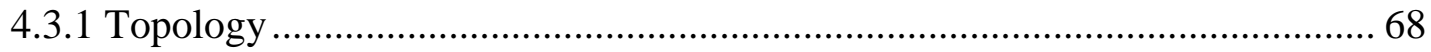

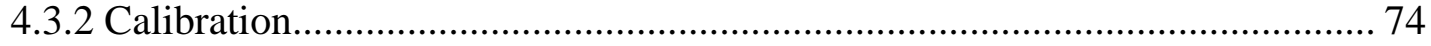

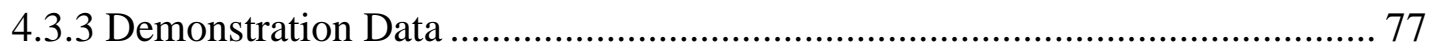

Chapter 5: Conclusion................................................ 83

5.1 Conclusion From Each Test........................................................................ 84

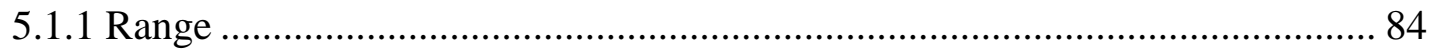

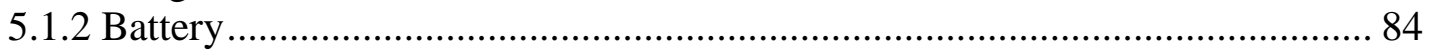

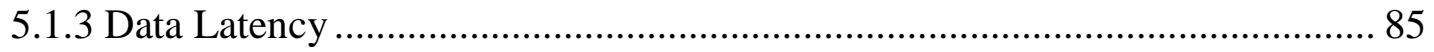

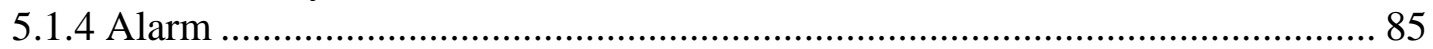

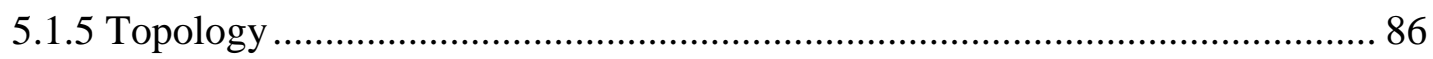

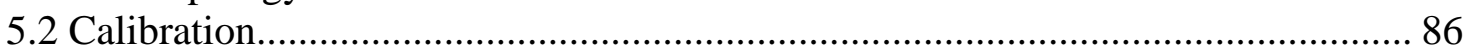

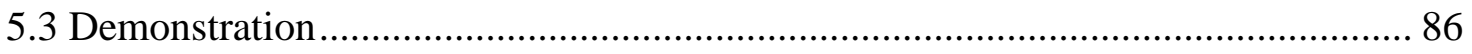

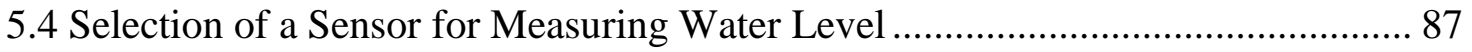

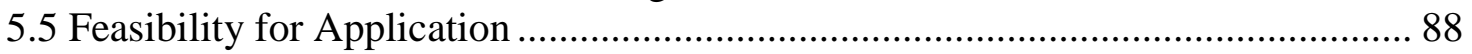

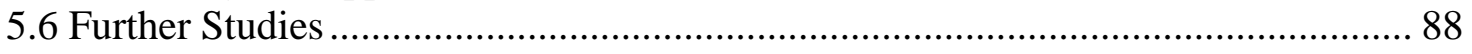

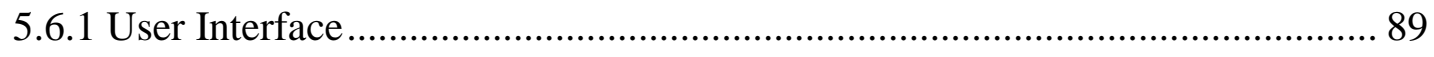

Bibliography ............................................................. 94

Appendix A (Raw Data)............................................... 97

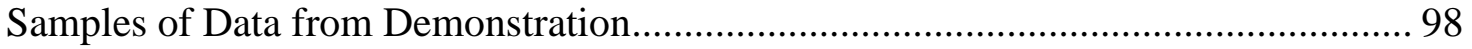

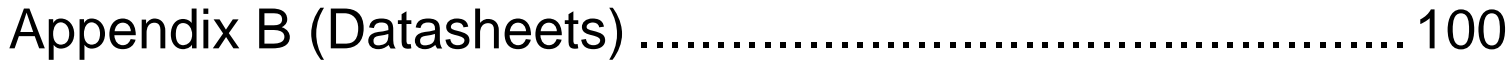




\section{List of Tables}

Table 2.1: Comparison of Zigbee Frequencies and Standard Frequency Ranges.....

Table 3.1: Technical Specifications of Zigbee Protocol

Table 3.2: Comparison of Specifications for Zigbee Components from Various Vendors

Table 3.3: Decision Matrix Used in Design of Network

Table 3.4: Comparison of Wired and Wireless Temperature Sensor Network Designs 32

Table 3.5: Node Health Table at 300m ... 35

Table 3.6: Excerpt from Spreadsheet of Battery Levels in Low-Power Mode ..... 38

Table 4.1: Percentage of Dropped Packets for Five Nodes at Various Distances

Table 4.2: Data Latency Results

Table 4.3: Calibration Data for P\&F Ultrasonic Proximity Sensor 


\section{List of Figures}

Figure 3.1: Crossbow IRIS Mote 2.4 GHz ...................................................... 25

Figure 3.2: Crossbow MTS400 Environmental Sensor Board .............................. 26

Figure 3.3: Crossbow MDA320 DAQ Board …………....................................... 27

Figure 3.4: Crossbow MIB520 USB Interface Board........................................... 28

Figure 3.5: Predicted Battery Life of Five Nodes Using Low-Power Mode.......... 38

Figure 3.6: Mesh Network Topology..................................................................... 43

Figure 3.7: Layout of Topology Test on Ground Floor of Engineering

Sciences Building ................................................... 45

Figure 3.8: Pepperl and Fuchs Ultrasonic Proximity Sensor..................... 47

Figure 3.9: American Sensor Technology Submersible Pressure Transducer... 48

Figure 3.10: Wiring Schematic CO Detector and Ultrasonic Proximity Sensor for

a Single Node

Figure 3.11: Unimeasure String Potentiometer ................................................. 52

Figure 3.12: Dwyer Non-submersible Pressure Transducer with LED Display .. 53

Figure 3.13: Wiring Schematic with Submersible and Non-Submersible Pressure Transducers, and String Potentiometer Sensor for a Single Node. 54

Figure 3.14: Layout of Power Supply Boxes ..................................................... 55

Figure 3.15: Wiring Schematic of Power Supply Boxes with CO Detector and Ultrasonic Proximity Sensor ................................................................ 57

Figure 3.16: Wiring Schematic of Power Supply Boxes with Pressure Transducers and String Potentiometer............................................... 58

Figure 3.17: Layout of Small-Scale Demonstration .............................................. 62

Figure 4.1: Batteries, Chargers, and Crossbow Module Completely Installed.... 64

Figure 4.2: Percentage of Dropped Packets for Five Nodes at Various Distances

Figure 4.3: Data Latency Test Results

Figure 4.4: Topology 1: Initial Setup with Nodes Transmitting on Best Available

Path

Figure 4.5: Topology 2: Simulated Node 1450's Destruction. Network SelfHealed by Rerouting Node 1451 through Node 1438

Figure 4.6: Topology 3: Node 1449 Selected a Better Data Path and Adaptively Rerouted through Node 1451.

Figure 4.7: Topology 4: Node 1450 Turned On and Routes Itself through Node 1400 .

Figure 4.8: Topology 5: Node 1450 Selects Direct Communication with the Base Station as the Best Data Path

Figure 4.9: Topology 6: Node 1451 Rerouted to Transmit through Node 1450.. 73

Figure 4.10: Topology 7: Node 1449 Selects Better Data Path and Reroutes

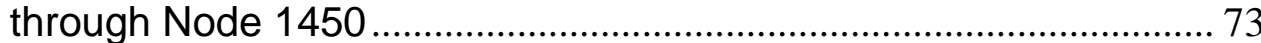

Figure 4.11: P\&F Ultrasonic Proximity Sensor Calibration Curve ......................... 76

Figure 4.12: Node 1438 Analog 0: String Potentiometer Data .............................. 78 
Figure 4.13: Node 1438 Analog 1: Non-Submersible Pressure Transducer

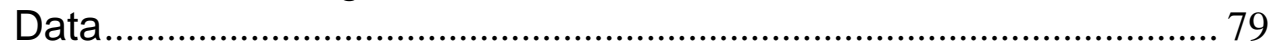

Figure 4.14: Node 1450 Pressure and Water Depth Data .................................... 80

Figure 4.15: Deflection and Pressure Data from Both Nodes................................ 81

Figure 4.16: All Node Data from Small Scale Demonstration................................. 82

Figure 5.1: Example of Operator Interface Under Normal Conditions.................. 90

Figure 5.2: Example of Operator Interface Under Flooding Conditions ............... 90

Figure 5.3: Detailed Data Display from Single Node with Alarm ........................... 91

Figure 5.4: Possible Actions to be Taken in the Event of an Emergency ............ 92 


\section{Chapter 1: Introduction}

\subsection{Overview}

A number of recent disasters in mines and tunnels have spurred interest in the development of systems for monitoring the health of an underground structure, and to detect the occurrence of accidents or acts of sabotage within the structure. The following list gives examples of recent mine/tunnel disasters and a brief description of each. While some of the disasters listed are better suited for a system as mentioned, all of these situations could have benefited in some way by having such a system.

- Sago Mine Disaster - Coal mine explosion, due to methane gas, on January 2, 2006 in Sago, West Virginia. Thirteen people were killed as a result of the disaster. In order to ensure their own safety, rescue teams had to continuously test for hazardous conditions such as mine seeps, explosive gas concentrations, and unsafe roof conditions [3].

- Quecreek Mine Disaster - A mine shaft flooded trapping miners 240 feet underground in June 2002 [4].

- London Subway Bombing - On July 7, 2005, suicide bombers attacked London's public transport system [15]. 
- Toronto Transit Commission - A train smashed into another train in the tunnel on August 12, 1995. Wreckage blocked air vents and temperatures rose over $40^{\circ} \mathrm{C}[6]$.

- Mont Blanc Tunnel - at least 35 people died as a result of a truck fire in the tunnel on March 24, 1999. Most of the fatalities resulted from poisonous gas emitted from the fire [18].

- Switzerland's Gotthard Tunnel - In October 2001, a fire due to a truck collision swept through the tunnel [5].

- Crandall Canyon Mine Disaster - In Huntington, Utah, a mine collapse killed 9 miners in August of 2007 [16].

As an additional example of the increasing interest in this area, the Mine Safety and Health Administration had this to say with regards to the implementation of technology in mine rescue efforts after the Sago Mine disaster.

Over the last several years, improvements have been made to communication devices, sensors, and other forms of technology in general industry. As such, continuous development and deployment of mine 
rescue equipment and technology are crucial to enhancing the effectiveness of mine rescue operations and improving miners' survivability in the event of a mine emergency [22].

With the disasters listed, as with any disaster, there is damage, and more than likely, there are fatalities or injuries. The design and implementation of a wireless sensor network was performed with the objective of mitigating the damage and injuries or deaths, with a particular interest in mitigating damage and mortality due to flooding. With the proper design and implementation of the monitoring network, this objective can be achieved by alerting the appropriate people of a damaging event, monitoring the conditions from the initial incident all the way to the rescue efforts, and providing additional actions in response to the event.

In order to be an effective solution to a problem with growing intrigue, the requirements of the intended network must be known and then be satisfied. For any given structure, coverage of the network must encompass all possible areas. With current technology, this requires a multi-node configuration. Due to the fact that it must be functional and still provide coverage following a damaging event, the network must be impervious to the loss of some sensors or nodes. The network must also be sufficiently redundant to ensure proper communication should a node fail or be destroyed. To add value, the monitoring network itself should be easily able to be retrofitted, and ideally, the network should be 
compatible with the existing security and communications systems. If these systems cannot be easily retrofitted to accommodate the monitoring system, it should be able to stand alone. The network should also be able to be easily scaled in order to fit any underground structure with the addition or subtraction of nodes. Finally, in order to be applied to various possible scenarios and structures, the sensors incorporated in the network must be customizable.

Once the requirements were understood for the network, the potential functions of a monitoring system were considered before design and implementation. One function of the network provides is aid in the detection and localization of an accident or incident. Another function is more rapid assessment of the situation surrounding the point of the occurrence and the progress of flooding or fire. If a monitoring network is implemented, the extent of the affected area and the condition or quality of air in the structure can be determined. The network may also be able to activate emergency lighting, escape aids, fire suppression equipment, or fire/flooding barriers.

\subsection{Motivation}

The interest and motivation behind the design and implementation of a monitoring network in mines or tunnels is to save lives and mitigate the damage to the affected structure. By monitoring conditions within a structure, a 
catastrophic event could potentially be stopped from occurring at all, thus saving lives and preventing damage.

Lives can be saved by a system of this nature in several additional ways. It provides warnings when hazardous conditions are present. Emergency beacons lighting escape routes or fire/flood suppression systems can be actuated by the system to protect any person that would have been affected. The system could also aid in rescue efforts and potentially save lives by finding people within a reasonable period of time.

As well as saving lives, the system can provide protection from damage to unaffected areas. Mechanical implementations interfaced with the network can be strategically located throughout the underground structure in an attempt to minimize damage to the infrastructure and the people affected. If damages can be confined to a small portion of a structure, the rest of the tunnel can be accessed to rescue people or assess the situation. 


\subsection{Objectives}

- Research on technologies for data acquisition and transmission in underground tunnels.

- Selection of design technology allowing the establishment of a "selfhealing" network able to cope with failure of one or more network nodes.

- Research and selection of a sensor suite for detecting explosions in tunnels and for assessing and monitoring conditions in the tunnel following such an event. Special attention paid to conditions leading to flooding of the tunnel.

- Development of a prototype sensor/data acquisition network and demonstration of same.

- Development of Human Interface software for analysis and display of data collected by the network.

- Development of plans for full-scale implementation and interface with public transportation infrastructure. 


\section{Chapter 2: Background}

\subsection{Introduction}

This project deals specifically with monitoring an underground structure, such as a mine or a tunnel, and controlling a mechanical device to contain flooding based on a detected breach in the mine or tunnel. The monitoring system must be designed as a network in order to completely monitor the structure. Based on the versatility of network designs, the final system may be adapted to monitor or control in applications other than the intended underground system.

\subsection{Wired vs. Wireless}

For the reasons of feasibility and implementation, the final design of this network prefers a wireless technology. Several drivers that create this preference include cost, feasibility, and physical structure. The network must provide complete and redundant coverage of the structure in order to sufficiently monitor all aspects of the mine or tunnel. This means that nodes will be placed in many locations and all require communication with the base station. In order to provide communication from one node to several others across an entire structure, a wire would have to span each desired connection. The cost and feasibility of using a 
wireless and wired network are discussed further in the technical approach. In addition to cost, providing redundant coverage of an entire structure means that nodes may be placed in various locations, but wiring will be installed in the same locations throughout the mine or tunnel. This means that a destructive event would disrupt communication to several nodes and more than likely sever the network causing a loss of coverage. Retrofitting a network for the application also becomes a point of interest and is investigated further.

Implementation of a wireless network, as opposed to a wired network, would alleviate the concerns that are raised with consideration of a wired network. A wireless network can provide sufficient redundant coverage without running cables to connect each node. Use of a wireless network can also ensure a line of communication throughout a structure even after a destructive event. Finally, the cost of installation or retrofitting becomes far less constricting.

\subsection{Network Technologies}

In researching and designing a network setup for this project, several different forms of networking were considered. These networking options included the following: Ethernet, Bluetooth, Wi-Fi (802.11b/g), and Zigbee (802.14.5). There are other networking options that were not researched because they do not pertain to the scope of this project. 


\subsubsection{Ethernet}

Ethernet, the common name for IEEE 802.3, is the dominant cabling and low level data delivery technology used in local area networks or LANS [23]. Ethernet typically transmits data at a rate of ten million bits per second; however, Fast Ethernet and Gigabit Ethernet can transmit at rates up to of $100 \mathrm{Mbps}$ and $1000 \mathrm{Mbps}$, respectively.

Consideration for Ethernet, though it may be fast and secure, stops at the fact that it utilizes wired connections and for the purpose of this project, a wireless network is preferred. Ethernet networks, LANs, also lack the capability for "self-healing." This means that if one node is destroyed or unable to transmit to other nodes, major portions of the network could be disabled, depending on how the network is configured. Ethernet was thus judged not to be appropriate for this application.

\subsubsection{Wireless Networks}

While the major drawback of a LAN is the fact that it must be hard wired, there are several wireless technologies that can be used as alternatives. Wireless communications offer users many benefits such as portability, flexibility, increased productivity, and lower installation costs [11]. 
Wireless networks can typically be placed into one of three categories based on their coverage ranges. The three categories are Wireless Wide Area Network (WWAN), Wireless Local Area Network (WLAN), and Wireless Personal Area Network (WPAN). An example of WWANs would be that of cellular/mobile phone coverage. WLANs are primarily Wi-Fi or IEEE 802.11. WPANs represent technologies such as Bluetooth, Infrared, and Zigbee. Wireless personal area networks (WPANs) are used to convey information over relatively short distances. Unlike wireless local area networks (WLANs), connections effected via WPANs involve little or no infrastructure [12]. WLAN and WPAN technologies have been determined to be relevant to the scope of this project.

Due to the fact that wireless networks use over the air communications, there are inherent security risks. Along with security risks, features of wireless networks, such as power consumption, range, data rate, and topology, begin carrying varying weights depending on the intended application.

\subsubsection{Wi-Fi (802.11)}

Wi-Fi, or Wireless Fidelity, is one of the most common WLANs and is governed by the IEEE 802.11 standard. The IEEE designed 802.11 to support medium-range, higher data rate applications, such as Ethernet networks, and to address mobile and portable stations [11]. Wi-Fi is nearly identical to an Ethernet or LAN except Wi-Fi institutes radio communication rather than wires to connect 
the access points. Wi-Fi operates at the frequency of $2.4 \mathrm{GHz}$ with varied data rates and corresponding ranges. The reliable coverage range for 802.11 WLANs depends on several factors, including data rate required, sources of RF interference, physical area and characteristics, power, connectivity, and antenna usage [11]. Theoretical ranges are from 29 meters (for $11 \mathrm{Mbps)}$ in a closed office area to 485 meters (for $1 \mathrm{Mbps}$ ) in an open area. However, through empirical analysis, the typical range for connectivity of 802.11 equipment is approximately 50 meters (about $163 \mathrm{ft}$.) indoors [11]. The potential range of more than 400 meters does not eliminate WLAN as a potential option to be implemented for this project; however, with such high data rates and large ranges, power consumption becomes a limiting factor in its consideration for this application.

\subsubsection{Bluetooth}

Bluetooth, an example of a WPAN, is a short range communication system intended to replace cable connections by use of wireless networking that can allow up to eight devices to be connected together in a mini-network called a piconet [10]. Bluetooth operates at the same frequency as Wi-Fi, $2.4 \mathrm{GHz}$, but transmits at a lower data rate of $1 \mathrm{Mbps}$. The operating range for Bluetooth is about 10 meters, but can be extended to reach up to 100 meters. When turned on, Bluetooth devices will automatically locate each other, but making connections with other devices and forming networks requires user interaction 
[11]. Bluetooth networks are considered to be ad hoc networks and are formed on a temporary and random basis by utilizing frequency hopping. By utilizing Frequency Hopping Spread Spectrum or FHSS, Bluetooth devices and networks change frequencies randomly up to 1600 times per second and are able to avoid interference with other devices operating on the $2.4 \mathrm{GHz}$ band.

Any time a connection is made between two or more Bluetooth devices, a piconet is formed. In a piconet, one device is the master and up to 7 other devices become the slave devices. The hopping sequence for a particular piconet is determined by the master's clock and address. A Bluetooth device can be a part of more than one piconet, but can only be a master in one. If several piconets combine, a scatternet is formed. Involvement in a scatternet does not necessarily imply any network routing capability or function in the Bluetooth enabled device. The Bluetooth core protocols do not, and are not intended to offer such functionality, which is the responsibility of higher level protocols and is outside the scope of the Bluetooth core specification [12].

In addition to having a robust network topology, Bluetooth devices consume relatively low amounts of power. Bluetooth specifications state a range of $30 \mu \mathrm{A}$ in a dormant mode to $8-30 \mathrm{~mA}$ in an active transmitting mode. Bluetooth chips also have excellent power-saving features, as they will automatically shift to a low-power mode as soon as traffic volume lessens or stops [10]. 
Bluetooth wireless technology offers several beneficial features such as a flexible network topology, low power, and low cost. Bluetooth is also omnidirectional and does not require line of sight in order to connect devices. Despite all of these benefits, range, required user action, and network scalability become an issue when looking at an application required for this project. Bluetooth technology also lacks the "experience" in an application like this as it is typically applied to connecting things like computers, PDAs, mobile phones, printers, and handsfree sets.

\subsubsection{Zigbee (802.15.4)}

Zigbee is the name of a specification of high level communication protocols that use small, low-power digital radios and are based on the IEEE 802.15.4 standard for wireless personal area networks. IEEE Std 802.15.4 defines the physical layer and medium access control sub layer specifications for low-data-rate wireless connectivity with fixed, portable, and moving devices with no battery or very limited battery consumption requirements typically operating in the personal operating space of $10 \mathrm{~m}$. Depending on the application, a longer range at a lower data rate may be an acceptable tradeoff [12]. The main objectives of an LR-WPAN or low rate wireless personal area network are ease of installation, reliable data transfer, short-range operation, extremely low cost, and a reasonable battery life, while maintaining a simple and flexible protocol 
[12]. The data transfer rate for an IEEE 802.145 network operating at a frequency of $2.4 \mathrm{GHz}$ is lower than that of Wi-Fi and Bluetooth at only $250 \mathrm{Kbps}$, but the maximum range extends past Wi-Fi and Bluetooth to about $500 \mathrm{~m}$.

An IEEE 802.15.4 WPAN operates in one of two topologies, a star topology or a peer-to-peer topology. In the star topology the communication is established between devices and a single central controller, called the PAN coordinator. The peer-to-peer topology is different from the star topology because any device can communicate with any other device as long as they are within range of one another. Peer-to-peer topology allows more complex network formations to be implemented, such as mesh networking topology [12]. A peer-to-peer network can be ad hoc, self-organizing, and self-healing. It may also allow multiple hops to route messages from any device to any other device on the network [12].

In many applications of the 802.15.4 standard, devices will be battery powered and battery replacement is impractical. For this reason, power consumption becomes an issue with these devices. Most Zigbee or 802.15.4 devices will spend a majority of time in a sleep state periodically listening for an RF signal to see if a message is pending. This mechanism allows the application designer to decide on a balance between battery consumption and message latency [12]. 
The application at hand requires several features of a wireless network to be considered including communication method, range, power consumption, topology, and data transmission rate. Each of these features carries a different weight and each wireless technology has its tradeoffs amongst the features. For this project, it was deemed that the network must be wireless and that range and power consumption were the two most important features. In comparing the different technologies, Wi-Fi and Bluetooth fall short of Zigbee in achievable range. Wi-Fi consumes much more power than both Bluetooth and Zigbee. Network topology also favors Bluetooth and Zigbee over Wi-Fi. Zigbee devices have a much slower data transfer rate than Bluetooth and Wi-Fi, but a high data rate is not a necessity for this application. With these comparisons, Bluetooth and Zigbee stand out as the better options, but a breaking point for Bluetooth is the limit of eight devices on a single piconet. Bluetooth also lacks the desired range.

Literature review and additional information led to the selection of the IEEE 802.15.4 standard protocol, more specifically Zigbee wireless technology, as the wireless transmission form. The Zigbee protocol is an ideal choice for this application. Zigbee provides a long range, low power, mesh network topology, and a sufficient data rate, $250 \mathrm{kbps}$, for the desired application. Zigbee devices also have the several important capabilities that are pertinent to the scope of this project. These capabilities include multi-hop, self-configuration, self-healing, and 
dynamic routing or frequency hopping. These are all very important software features of Zigbee to go along with the satisfactory hardware specifications.

\subsection{Applications}

\subsubsection{Wireless in Mines}

Consideration is being given to replacing existing wired systems with wireless ones and consideration is being given to the potential for new applications of wireless networks. For instance, with the passing of the Mine Improvement and New Emergency Response Act or MINER Act in 2006, safety regulations are calling for wireless monitoring/tracking and wireless communication methods to be enacted.

"POST-ACCIDENT COMMUNICATIONS.--The plan shall provide for a redundant means of communication with the surface for persons underground, such as secondary telephone or equivalent two-way communication.

POST-ACCIDENT TRACKING.--Consistent with commercially available technology and with the physical constraints, if any, of the mine, the plan shall provide for above ground personnel to determine the current, or immediately pre-accident, location of all underground personnel. Any 
system so utilized shall be functional, reliable, and calculated to remain serviceable in a post-accident setting [21].

The MINER Act also states that there must be mine-wide coverage and the system must be available post disaster. A properly designed Zigbee mesh network would be an ideal solution to this problem by providing mine-wide coverage, possibly redundant coverage in areas, and by having the ability to selfheal and thus to be available following the destruction of a node. More research is currently being conducted regarding various methods of communication and tracking that could satisfy the conditions in the MINER Act.

In general, wireless communications are a benefit because they allow communications throughout the mine without wires; however, wireless communications become a challenge in underground coal mines due to radio signal propagation. Radio frequencies such as very high frequency (VHF) and ultra high frequency (UHF) can suffer both attenuation and severe corner losses [20]. Tests have shown that the mine entry acts as a wave guide to help propagate signals, but the system must be modified in the mine to help propagate the signals mine-wide. Some systems either use a Leaky Feeder or a wireless mesh to do this. The wireless mesh involves installing signal repeaters or "nodes" throughout the mine. These nodes pass communications from one another to get the communications from the mine [20]. Table 2.1 shows a 
comparison of the frequency ranges for the mentioned frequencies as well as frequencies pertaining to the various Zigbee data rates.

Table 2.1: Comparison of Zigbee Frequencies and Standard Frequency Ranges

\begin{tabular}{|c|c|}
\hline Type of Frequency & Frequency Range \\
\hline Extra Low (ELF) & $3 \mathrm{~Hz}-3 \mathrm{kHz}$ \\
\hline Very Low (VLF) & $3 \mathrm{kHz}-30 \mathrm{kHz}$ \\
\hline Low (LF) & $30 \mathrm{kHz}-300 \mathrm{kHz}$ \\
\hline Medium (MF) & $300 \mathrm{kHz}-3 \mathrm{MHz}$ \\
\hline High (HF) & $3 \mathrm{MHz}-30 \mathrm{MHz}$ \\
\hline Very High (VHF) & $30 \mathrm{MHz}-300 \mathrm{MHz}$ \\
\hline Ultra High (UHF) & $300 \mathrm{MHz}-3 \mathrm{GHz}$ \\
\hline \multirow{3}{*}{ Zigbee Frequencies } & $2.4 \mathrm{GHz}$ \\
\hline & $915 \mathrm{MHz}$ \\
\hline & $868 \mathrm{MHz}$ \\
\hline
\end{tabular}

Extremely low frequency (ELF), very low frequency (VLF), and low frequency (LF) suffer less attenuation, but can experience electrical interference from motors and other equipment [20]. Medium frequency (MF) has less severe attenuation characteristics than VHF and UHF signals and does not require a leaky feeder cable [20]. Instead, MF uses parasitic propagation effects to send the signal over distances up to two miles. Parasitic propagation uses metal objects such as wires, pipes, and rails in the mine to propagate the signal. Combining the attributes of the various wireless frequencies and systems could provide a survivable mine-wide wireless communications systems but would require that the systems are interoperable [20]. 
In deciding on a technology or network of different technologies, attention needs to be paid to the issue of miner tracking. In the event of an emergency, miner tracking systems will be integrated with the communication network using wireless communications technology. There are three types of tracking systems [20]. In the first system, zone or proximity based, miners wear active batterypowered or passive radio-frequency ID (RFID) tags or similar technology to identify themselves as they pass readers placed at intersections within the mine [20]. Radio location node-based technologies require that miners carry a radio device that communicates with radio nodes. Location is determined by identifying the nodes with which the miner can communicate. Resolution is dependent on both the number of nodes and the means of signal processing. This type of tracking is somewhat inherent to wireless mesh communications systems [20]. Zone based and radio based location technologies are both commercially available and designs have been submitted for mining applications. The third tracking technology, infrastructure "autonomous" system, however is not widely available and in many cases is pushing state-of-the-art. In an infrastructure "autonomous" system, miners wear a device that determines the location independent of any active elements in the mine [20]. 


\subsection{Conclusion}

Despite the relative need for a technology in a select corner or the market, industry has been, and always will be, the driving force behind development of emerging technologies such as wireless networks. Some industries are beginning to see the value in wireless monitoring systems and even considering wireless control systems. There is still the issue of an industry wide standard for wireless mesh networks though. While some companies have formed groups and agreed upon certain specifications, such as WirlessHART or ISA100.11a, efforts are still underway to develop or agree upon a standard for wireless sensor networks in industry. This would allow hardware from different companies to be used in a single network and be guaranteed interoperability by the standard. The growing interest in wireless sensor networks has led to the development of many new technologies and is leading to the implementation of a standard for wireless sensor networks and a greater variety of applications. 


\section{Chapter 3: Technical Approach}

The scope of this project requires the design, construction, and implementation of a monitoring/control system. The monitoring portion of the system possesses a number of built-in sensors and capabilities as well as the ability to customize the system by adding sensors. The control system is entirely customizable depending on the application. With the given hardware, digital outputs can be used to control a number of helpful devices in the event of a disaster or presence of hazardous condition. If an event or condition occurs, that is not readily detectable by an operator or other people involved, the wireless network can be used to turn on warning lights or sound an alarm to notify anybody that would be affected. The system could also be used to activate guide beacons to show the exit for potential survivors. Actuating a fire suppression system or mechanical suppression system are also potential uses of the wireless system.

\subsection{Selection of Zigbee Protocol}

With the desire of a wireless mesh network to be the topology used, the Zigbee protocol was selected as it is the best fit for this application.

Zigbee technology in itself has many features that allow for flexible and straightforward implementation. These features include multi-hop, self- 
configuration, self-healing, and dynamic routing or frequency hopping. Multi-hop is the capability of sending messages from a node through one or more other nodes before reaching the base station. Self-configuration is the capability of the network to form without human intervention and reconfigure itself when a node is added or removed. Self-healing is the capability to reroute a signal to a functional node if one or more nodes is destroyed or fail without resetting the entire network.

The more technical specifications of the Zigbee protocol are shown by the following table.

Table 3.1: Technical Specifications of Zigbee Protocol

\begin{tabular}{|c|c|}
\hline \multicolumn{2}{|c|}{$\begin{array}{c}\text { Specifications for Zigbee Protocol } \\
\text { (Some ranges depend on Vendor) }\end{array}$} \\
\hline Data Rates & $250 \mathrm{~kb} / \mathrm{s}, 100 \mathrm{~kb} / \mathrm{s}, 40 \mathrm{~kb} / \mathrm{s}$, and $20 \mathrm{~kb} / \mathrm{s}$ \\
\hline $\begin{array}{c}\text { Channels } \\
\text { (Frequencies } \\
\text { of Transmission) }\end{array}$ & $\begin{array}{c}16 \text { in the } 2450 \mathrm{MHz} \text { band, } \\
30 \text { in the } 915 \mathrm{MHz} \text { band, } \\
\text { and } 3 \text { in the } 868 \mathrm{MHz} \text { band }\end{array}$ \\
\hline Range & $\begin{array}{c}50 \mathrm{~m} \text { to } 500 \mathrm{~m} \text { typical } \\
\text { (varies depending on vendor and } \\
\text { environment) }\end{array}$ \\
\hline Topologies & $\begin{array}{c}\text { Star } \\
\text { Peer-to-Peer } \\
\text { Mesh }\end{array}$ \\
\hline Battery Life & $\begin{array}{c}\text { Months to Years } \\
\text { (depending on activity and hardware used) }\end{array}$ \\
\hline $\begin{array}{c}\text { Number of } \\
\text { devices }\end{array}$ & $\begin{array}{c}1.845 \times 10^{19} \text { devices (64 bit IEEE } \\
\text { address) } \\
(65,535 \text { networks) }\end{array}$ \\
\hline
\end{tabular}


Highlights from the table include multiple channels at each frequency, allowing for frequency hopping in the network to avoid interference. Also, the upper value for the range, $500 \mathrm{~m}$, and the availability of the mesh topology makes the Zlgbee Protocol more applicable for structures such as mines or tunnels. The number of devices that are supported in a single network allow for the network to be scaled to fit a variety of structures.

\subsubsection{Selection of Vendor}

In addition to the functionality that comes with the Zigbee wireless technology, the Zigbee family of devices has many design options as well. With a wide array of companies each producing their own species of Zigbee devices, there are many options for implementing this network. Several products from various vendors were researched and the relevant features of each product were compared. The technical specifications and features of the hardware such as range, power consumption, cost, and overall functionality were determined to be the relevant features for the implementation of a wireless network in an underground structure. The table below shows a comparison of the technical specifications for the Zigbee component that each vendor offers. 
Table 3.2: Comparison of Specifications for Zigbee Components from Various Vendors

\begin{tabular}{|c|c|c|c|c|c|c|c|}
\hline Vendor & $\begin{array}{l}\text { Name of } \\
\text { RF Module }\end{array}$ & Cost & $\begin{array}{c}\text { Power } \\
\text { Consumption }\end{array}$ & Range & $\begin{array}{l}\text { Data } \\
\text { Rate }\end{array}$ & $\begin{array}{l}\text { Topology/ } \\
\text { Self-Healing }\end{array}$ & $\begin{array}{l}\text { Reason for } \\
\text { Elimination }\end{array}$ \\
\hline Xbee [24] & $\begin{array}{c}\text { XBee-PRO } \AA \\
\text { ZB }\end{array}$ & $\begin{array}{l}\$ 34 \\
\text { ea. }\end{array}$ & 45-295 mA @ 3.3 V & $\begin{array}{c}120- \\
1600 \mathrm{~m}\end{array}$ & $\begin{array}{l}250 \\
\text { kbps }\end{array}$ & Mesh/Yes & Chip level \\
\hline $\begin{array}{l}\text { Helicomm } \\
\text { [8] }\end{array}$ & $\begin{array}{l}\text { IP-Link1220- } \\
2133 \\
\text { Embedded } \\
\text { Module }\end{array}$ & $?$ & $\begin{array}{l}\text { 43-50 mA Receive } \\
\text { 37-290 mA Transmit }\end{array}$ & $\begin{array}{c}100- \\
1200 \\
m\end{array}$ & $\begin{array}{l}250 \\
\text { kbps }\end{array}$ & Mesh/Yes & $\begin{array}{l}\text { Communic- } \\
\text { ations only }\end{array}$ \\
\hline $\begin{array}{c}\text { Crossbow } \\
{[14]}\end{array}$ & IRIS 2.4GHz & $\begin{array}{l}\$ 115 \\
\text { ea. }\end{array}$ & $\begin{array}{c}18-25 \text { mA active @ } 2.7 \\
\mathrm{~V}-3.3 \mathrm{~V}\end{array}$ & $\begin{array}{l}\text { Up to } \\
500 m\end{array}$ & $\begin{array}{l}250 \\
\mathrm{kbps}\end{array}$ & Mesh/Yes & \\
\hline Telegesis [2] & ETRX2 & $\begin{array}{l}\$ 40.77 \\
\text { ea. }\end{array}$ & ? mA @ 2.1 to 3.6V & $?$ & $\begin{array}{l}250 \\
\mathrm{kbps}\end{array}$ & Mesh/Yes & Chip level \\
\hline RFM [25] & Any & $?$ & 25-130 mA @ 0 to 3.3 V & $?$ & $\begin{array}{l}250 \\
\mathrm{kbps}\end{array}$ & Mesh/Yes & Chip level \\
\hline One RF [19] & $\begin{array}{c}\text { TinyOne } \\
\text { ZigBee } 2.4 \\
\text { GHz }\end{array}$ & $?$ & 30 mA @3.6 V & $\begin{array}{l}\text { Up to } \\
70 \mathrm{~m}\end{array}$ & $\begin{array}{l}250 \\
\text { kbps }\end{array}$ & Mesh/Yes & Chip level \\
\hline
\end{tabular}

As shown in the table, the best fit for this project and demonstration purposes is Crossbow. The primary form of the components offered by nearly all of the vendors found was on a basic chip level. The chip level radio modules could have been used, but it was determined that for this project, a more functional component was needed that would allow for implementation of a sensor suite to be used in the wireless sensor network. Crossbow's $2.4 \mathrm{GHz}$ IRIS module has the lowest power consumption out of all of the radio modems, has a sufficient range for many applications, and uses a mesh topology and is therefore a self-healing network. Cost of the selected hardware was considered, but was the least important factor in deciding the vendor. All of the factors were given weighting factors as shown in the System Design section and 
consideration was given to all of the vendors, but ultimately Crossbow was selected as the vendor.

\subsubsection{Hardware Selection}

After selecting Crossbow as a vendor, specific products were selected from Crossbow and purchased to build a test network and perform a small scale demonstration.

The Crossbow IRIS $2.4 \mathrm{GHz}$ radio module was selected as the device with which to build the network. The radio module is shown below along with a brief description of its functions.

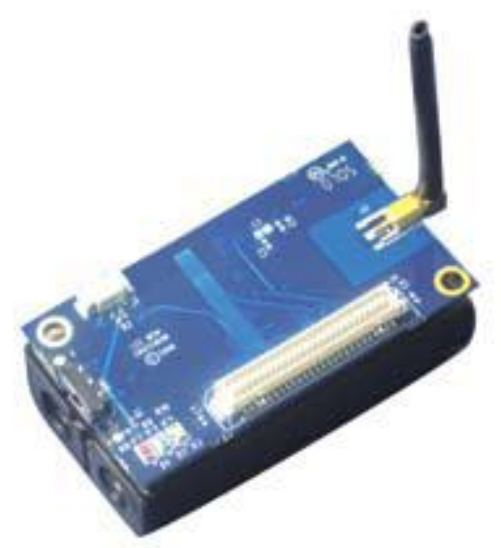

Figure 3.1: Crossbow IRIS Mote $2.4 \mathrm{GHz}$

The IRIS module operates as an IEEE 802.15.4 compliant RF transceiver. The module allows for ranges as far as $\mathbf{5 0 0}$ meters to be covered between nodes 
without amplification. In order to avoid possible interference issues, direct sequence spread spectrum radio is used and provides inherent data security. Each IRIS mote has router capabilities allowing it to transmit as well as receive data. This allows for a scalable mesh network to be formed. All of these features are sufficient for the network designed and are the reasons for the selection of this specific piece of hardware.

In order to monitor standard environmental conditions, a sensor board was selected from Crossbow that directly interfaces with the IRIS module via a 51-pin connector. The MTS 400 was the selected sensor board and is shown below.

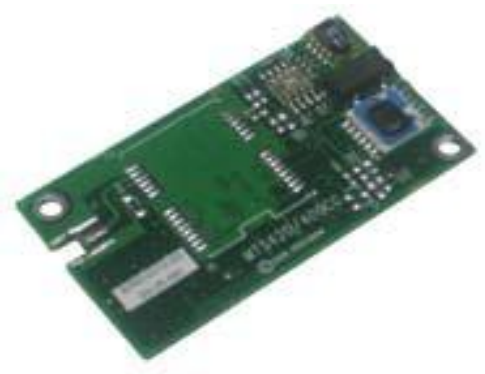

Figure 3.2: Crossbow MTS400 Environmental Sensor Board

The MTS 400 has onboard sensors including a dual-axis accelerometer, a barometric pressure sensor, an ambient light sensor, a relative humidity sensor, and a temperature sensor. These are all useful sensors for the given application and will provide important data pertaining to the surrounding environment. 
Along with monitoring the environmental conditions, other conditions such as air quality(CO content, smoke content), water depth, in case of flooding, and location of equipment or people in the structure are to be monitored. In addition to the need to interface sensors to monitor these conditions, an interface must be provide in order to control emergency systems such as warning lights, guide beacons, audio enunciators, or fire suppression equipment. In order to interface the IRIS module with all of these possibilities, a data acquisition board with analog I/O and digital I/O was selected. The MDA320 DAQ board, shown below with a description of relevant features, allows for a variety of digital or analog sensors and control systems to be added to the wireless network as needed.

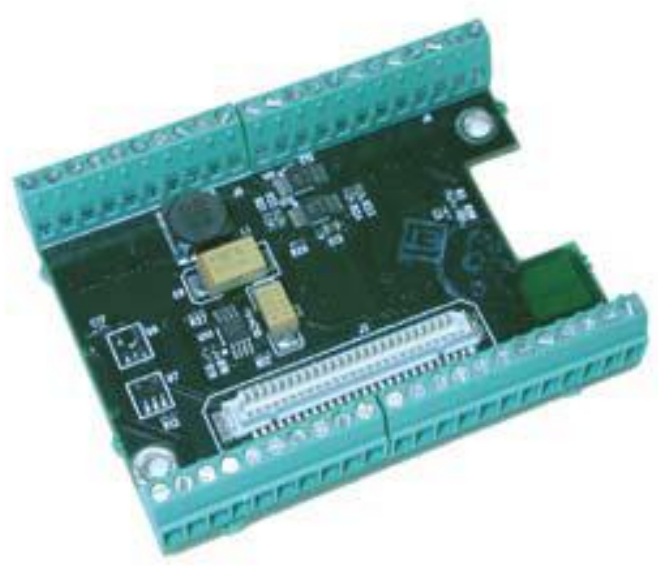

Figure 3.3: Crossbow MDA320 DAQ Board

The micro-terminals on the MDA 320 board allow for up to 8 channels of 16 bit analog input and 8 digital I/O channels to be connected to the board and transmitted through the IRIS module. The MDA board connects to the IRIS 
module via the 51-pin connector and has an additional 51-pin connector to which the MTS environmental board can be attached.

The wireless modules and sensor boards will be connected together and dispersed in strategic locations for monitoring. The interface with the computer, acting as a base station, is achieved using the MIB520 USB Interface Board. This board acts as a gateway to the computer by receiving the signals from the nodes and displaying the data using the software provided. The MIB520 board is shown below.

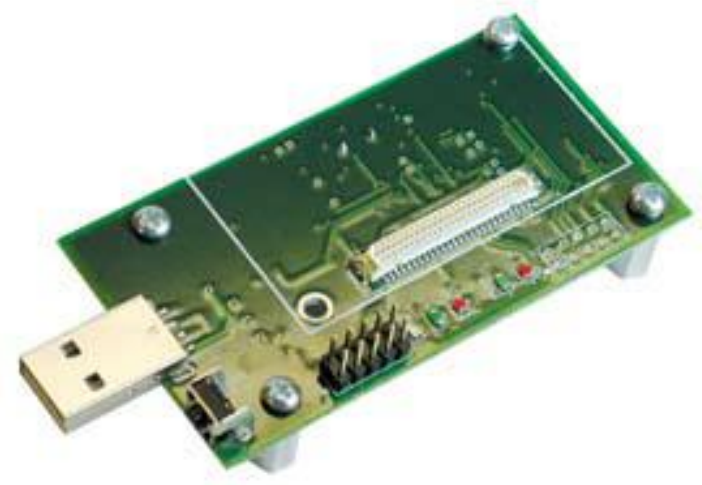

Figure 3.4: Crossbow MIB520 USB Interface Board

The IRIS motes and MTS400 boards purchased from Crossbow can be set up to form a basic wireless sensor network, and the MDA320 board allows for 
a variety of sensors to be added to the network. The array of possible sensors is customizable and provides versatility to the network so that it could be used in many different applications.

\subsection{Problem Statement}

The eventual implementation of a wireless network requires that the network be available at all times, provide complete, redundant coverage of the monitored structure while monitoring all necessary conditions, and be operational following a destructive event within the mine or tunnel.

To be more specific, the system is required to monitor conditions under "normal" circumstances and be able to detect an "abnormal" event. "Normal" conditions can be described as the standard operating conditions for the given structure and procedures going on within the structure. These conditions will be used to determine a threshold within which the given sensor outputs must fall. Specific conditions that will be monitored include environmental conditions such as temperature and humidity, air quality such as smoke or carbon monoxide content, and water levels.

Any sensor readings that fall outside of the desired threshold are deemed as "abnormal" conditions and an operator will be notified of such an event. Potential causes for abnormal conditions can include an explosion, collapse of a 
tunnel, fire, or flooding. Abnormal conditions can not be limited to these causes, as they are just examples, but can be described more in general as any potentially hazardous condition or event that requires evacuation or other emergency measures to be implemented. In order to track these conditions, a sensor suite was designed to be attached to the network.

\subsection{System Design}

The system design consists of two sections. The first and main section was the design of a wireless network. The second section was the selection of a sensor suite to be connected to the network.

\subsubsection{Wireless Network Design}

The constraints that were used in selection and design of the wireless network were cost, range, power consumption, topology, and data transmission rate. Each of these factors carries a different weight for the given application, but the most important requirement is that the wireless network must be able to reconfigure or "self-heal" following any damage to the network or surrounding area. This was determined to be a function of given network topologies and was considered along with topology in selecting a network. The decision matrix below shows the weighting factors assigned to each feature, illustrating the relative importance of each. 
Table 3.3: Decision Matrix Used in Design of Network

\begin{tabular}{|c|c|c|c|c|c|}
\cline { 2 - 5 } \multicolumn{1}{c|}{} & Cost & Range & $\begin{array}{c}\text { Power } \\
\text { Consumption }\end{array}$ & $\begin{array}{c}\text { Data } \\
\text { Transmission } \\
\text { Rate }\end{array}$ & $\begin{array}{c}\text { Topology/ } \\
\text { Self- } \\
\text { Healing }\end{array}$ \\
\hline $\begin{array}{c}\text { Weighting } \\
\text { Factor }\end{array}$ & 4 & 8 & 6 & 5 & 10 \\
\hline
\end{tabular}

\subsubsection{Cost}

Experts agree that while mobility and modularity of a wireless system are important in some cases, the cost and ease of installation for a wireless network are the driving forces behind implementation of a wireless system rather than a wired system [1]. The cost for sensors and signal conditioning hardware may be higher for a wireless network, but after accounting for the cost of wire and installation for a wired network a wireless network can be less expensive.

In a demonstration performed by PNNL, Pacific Northwest National Lab, an in-building temperature sensor network was implemented and the cost of a wired network and wireless network were each estimated. Eighteen AWG cable was assumed for sensor connections at an approximate cost of $\$ 0.07 / \mathrm{ft}$. and a labor cost of $\$ 1.53$ per linear foot of wiring (RS Means 2001) [1]. The table below shows a break down in the cost of each component used, total cost, and average cost per sensor for the application that PNNL demonstrated. 
Table 3.4: Comparison of Wired and Wireless Temperature Sensor Network Designs

\begin{tabular}{|c|c|c|}
\hline \multirow[b]{3}{*}{ Cost Component } & \multicolumn{2}{|l|}{ Cost } \\
\hline & \multicolumn{2}{|c|}{$\begin{array}{l}\text { In-Building } \\
\text { Temperature } \\
\text { Sensor Network }\end{array}$} \\
\hline & $\begin{array}{l}\text { Wired } \\
\text { Design }\end{array}$ & $\begin{array}{l}\text { Wireless } \\
\text { Design }\end{array}$ \\
\hline Sensors & $\$ 1,800$ & $\$ 3000^{[1]}$ \\
\hline Wiring & $\$ 4800^{[2]}$ & -- \\
\hline $\begin{array}{l}\text { Communication } \\
\text { and signal-conditioning } \\
\text { hardware }\end{array}$ & -- & 2475 \\
\hline Labor & $-{ }_{-[}^{[3]}$ & 800 \\
\hline Total cost & 6600 & 6275 \\
\hline Average cost per sensor & 220 & 209 \\
\hline
\end{tabular}

[1] Temperature sensors each with an integrated transmitter. [2] Including labor for installation.

[3] Included in cost of wiring.

Along with the initial cost of installation being an issue in comparing wired to wireless networks, retrofitting a system also serves as a selling point. Installation in a new structure may favor a wired network slightly due to ease of access throughout the structure; however, retrofitting a system in an existing structure favors a wireless network because of the lack of access to certain points and the potential obstruction of current operations. In the case of a mine or tunnel, operations and traffic through the structure would have to be completely stopped for the implementation of a wired network. On the other hand, a wireless network would allow for modules to be installed one at a time, 
only affecting a minimal area of operation. By having to shut down or limit operations in order to install wiring, more money is lost due to the down time experienced. This opportunity cost of operation can also be considered in weighing a wireless network versus a wired network.

For the wireless system designed for this project, a mesh network is to be used which creates an additional selling point for the use of a wireless network. The mesh network contains router nodes, nodes that transmit as well as receive data. This allows for infinite connectivity with no additional infrastructure in a wireless network, whereas in a wired network, a wire would have to span each desired connection.

For the reasons of cost and ease of installation, as well as survivability and modularity, it was determined that a wireless mesh network would be more favorable than a wired network. However, the selection of a wireless network required additional research and design work to be performed.

\subsubsection{Range}

Range testing was also required in designing the network because it showed the maximum range at which the node will provide satisfactory transmissions. The test also showed how the distance affects the health of the nodes. This is important because the information gathered will be pivotal in 
determining the distances between the nodes in actual implementation to achieve sufficient redundancy. The range test also helps in determining the "sufficient redundancy" for the system.

The range test was performed by first selecting an area with suitable line of sight in order to test the full range of the nodes. Distances were measured using a laser range finder and then marked at intervals of 50 meters starting at 300 meters. Five nodes were then placed at the $\mathbf{3 0 0}$ meter mark. It was determined that the nodes should be reliable at 300 meters since they are quoted to have a range of 500 meters. The nodes were then turned on and allowed to transmit several health packets to the base station. An example of the data transmitted in the health packets is shown below in Table 3.5. The column titles in Table 3.5 are defined in the list below.

- Health Packets - the total number of node health and neighbor health packets that have originated at the node after the last reboot.

- Node Packets - the total number of packets that have originated at the node since the last reboot. This number includes all data, node health, neighbor health and route update packets.

- Forwarded Packets - the total number of packets that the node has forwarded from other nodes. 
- Dropped Packets - the total number of packets that the node has dropped. Packets are considered to be dropped when 1 packet has been retransmitted 8 times without receiving acknowledgement.

- Retries - the total number of retransmissions that the node had to make due to the fact that it did not receive acknowledgement.

- Battery Voltage - a measure of the battery voltage available to the Mote.

Table 3.5: Node Health Table at 300m

Node Health
\begin{tabular}{lllllllll} 
Id $\Delta$ & health_pkts node_plts forwarded dropped & retries & battery \\
\hline 1445 & $0.94 \%$ & $32.39 \%$ & $65.96 \%$ & $1.64 \%$ & $48.83 \%$ & $2.6 \mathrm{v}$ \\
1449 & $2.74 \%$ & $95.21 \%$ & $0 \%$ & $4.79 \%$ & $32.19 \%$ & $2.5 \mathrm{v}$ \\
1450 & $1.86 \%$ & $63.72 \%$ & $32.56 \%$ & $3.72 \%$ & $87.91 \%$ & $2.6 \mathrm{v}$ \\
1451 & $0.59 \%$ & $20.36 \%$ & $79.2 \%$ & $0.45 \%$ & $0.45 \%$ & $2.6 \mathrm{v}$ \\
2206 & $1.49 \%$ & $51.87 \%$ & $43.66 \%$ & $4.48 \%$ & $77.99 \%$ & $2.5 \mathrm{v}$ \\
\hline
\end{tabular}

After the data was recorded, the nodes were turned off. The database was then reset and the entire process was repeated at 50 meter increments up to 500 meters. The entire process was performed with five nodes to test for possible variances in the nodes. The results from each distance were recorded and interpreted in the Results section. 
Some of the data in Table 3.5 and in the range test overall was slightly skewed due to the fact that the base station did not begin receiving and recording data until the nodes had been transmitting for a short period of time. This caused the percentages to be higher in the beginning of the test and a larger variation amongst the nodes at each distance. The percentages began falling as time passed to steady the data. If the data was recorded for a longer period of time, the beginning portion of each distance would have been made to be less significant to the overall data. As a result, the data would have been more reliable. However, testing for a longer period of time was not feasible for the given location.

The delay from the time the nodes were turned on to the time the base station began recording should be relatively consistent for all five distances. This means the data should be equally skewed for all five cases and still be able to be used in a legitimate comparison, shown in the results.

\subsubsection{Power Consumption}

Power consumption by the nodes was incorporated in the system design in order to determine if a feasible timeframe could be achieved as quoted by the vendor. The selected wireless system can operate on two modes, high power and low power. In order to test the battery life of each node, a simple network 
was set up using each mode of power to determine how long two AA batteries could sustain the wireless module as well as an environmental sensor board.

The high power mode was tested first. By default this mode reports data from each node at eight second intervals. During the testing, after a battery level of approximately 1.8 volts was reached and the sensor board did not have enough power to support the onboard sensors. The sensors then failed to provide accurate data. For example, the temperature sensor output values that were hundreds of degrees from the actual temperature. An output value such as this could set off a false alarm, but can be accounted for by monitoring the voltage of the nodes in conjunction with the sensor data. An eight second time interval was used until the data returned to the base station was unusable. The results showed that the battery life of two AA batteries in high power mode was five to six days.

The low power mode was then tested using the same network. The low power mode has a default time interval of 3 minutes between data readings. Using this interval, the sensor board was sustained for a one month period while the data was recorded. After this time period, the nodes were still operating so the data was taken from each node, placed into a spreadsheet program and a trend line was used to determine the predicted life of the batteries. The trend line gives an estimated life of 3.5 to 4 months. This data is show in the table below and illustrated in Figure 3.5. 
Table 3.6: Excerpt from Spreadsheet of Battery Levels in Low-Power

Mode

\begin{tabular}{|c|c|c|c|c|c|}
\hline & $\begin{array}{l}\text { Node } \\
1451\end{array}$ & $\begin{array}{l}\text { Node } \\
1450\end{array}$ & $\begin{array}{l}\text { Node } \\
2206\end{array}$ & $\begin{array}{l}\text { Node } \\
1449\end{array}$ & $\begin{array}{l}\text { Node } \\
1445\end{array}$ \\
\hline Time & Voltage & Voltage & Voltage & Voltage & Voltage \\
\hline 4/14/2008 23:32 & 2.717 & 2.7585 & 2.7464 & 2.7284 & 2.7585 \\
\hline 4/15/2008 0:00 & 2.7166 & 2.7464 & 2.7464 & 2.7284 & 2.7524 \\
\hline 4/15/2008 12:00 & 2.7049 & 2.7225 & 2.7344 & 2.7284 & 2.7524 \\
\hline 4/16/2008 0:00 & 2.6932 & 2.7107 & 2.7284 & 2.7225 & 2.7344 \\
\hline 4/16/2008 12:00 & 2.676 & 2.699 & 2.7225 & 2.7166 & 2.7344 \\
\hline 4/17/2008 0:00 & 2.6703 & 2.6703 & 2.7166 & 2.7107 & 2.7284 \\
\hline 4/22/2008 2:32 & 2.5822 & 2.5929 & 2.631 & 2.62 & 2.6421 \\
\hline $4 / 22 / 2008$ 12:01 & 2.5875 & 2.6036 & 2.6365 & 2.631 & 2.6477 \\
\hline 4/22/2008 23:59 & 2.5875 & 2.6036 & 2.6365 & 2.631 & 2.6421 \\
\hline 4/23/2008 12:00 & 2.5875 & 2.6036 & 2.631 & 2.62 & 2.6421 \\
\hline 4/23/2008 23:59 & 2.5822 & 2.5982 & 2.631 & 2.62 & 2.6421 \\
\hline 4/24/2008 11:59 & 2.5822 & 2.5982 & 2.62 & 2.62 & 2.6365 \\
\hline 4/24/2008 21:05 & 2.5769 & 2.5982 & 2.62 & 2.6145 & 2.631 \\
\hline 4/26/2008 12:00 & 2.561 & 2.5929 & 2.6091 & 2.6091 & 2.62 \\
\hline $4 / 27 / 200823: 59$ & 2.5558 & 2.5875 & 2.6091 & 2.5982 & 2.6145 \\
\hline $4 / 28 / 200811: 59$ & 2.5558 & 2.5875 & 2.6036 & 2.5982 & 2.6145 \\
\hline
\end{tabular}

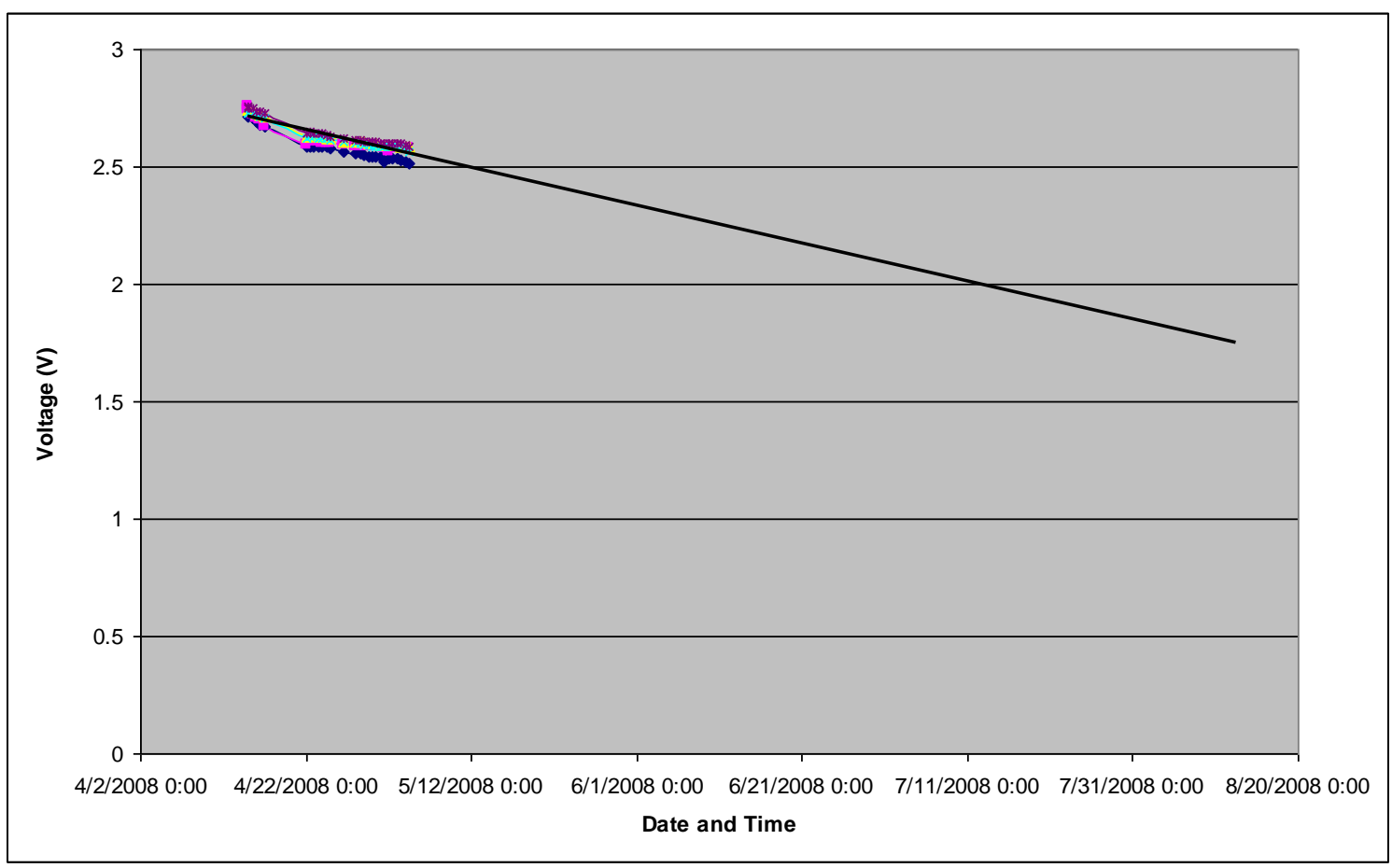

Figure 3.5: Predicted Battery Life of Five Nodes Using Low-Power Mode 


\subsubsection{Data Latency}

The next design test consisted of timing the latency of a node as it reconnected with the base station after being rebooted. This test was used to determine if the network reconfiguration time was low enough to satisfactorily monitor a given structure. The data latency test was performed by placing a node where it was confirmed that it could not communicate with the base station

directly. A node was then placed at an intermediate location allowing data to be transmitted from the first node, through this node, and on to the base station. Once this setup was confirmed, a test of the time it takes for the network to reconfigure was performed. This was done by turning off the intermediate node, confirming again that the first not could not communicate with the base station, and then turning the intermediate node back on. The time for the node to transmit its first packet and the packet of the farthest node were both recorded and analyzed.

\subsubsection{Sensor Suite Selection}

The conditions that are required to be monitored within the underground structure govern the selection of a sensor suite. For this network, the basic sensor suite consists of the MTS400 environmental board. This sensor board allows for standard environmental/atmospheric conditions (temperature, 
pressure, humidity, and ambient light) to be monitored on a semi-continuous basis.

Expanding on the basic sensor suite is done through the MDA320 DAQ boards. Adding external sensors through the DAQ board allows for a customizable wireless sensor network to be created and meet the needs of many various scenarios. With the focus of mitigating damages due to flooding, additional sensors were selected to be used to monitor water levels. Additional sensors were also incorporated to show the versatility of the network and to be used in a small scale demonstration.

The sensors that were selected to be connected to the Crossbow hardware were intended to monitor water level and CO content. These measurements were accomplished using an ultrasonic proximity sensor, submersible and non-submersible pressure transducers, and a $\mathrm{CO}$ detector.

Once connected, the environmental sensors and external sensors will provide baseline data readings. These readings allow for a range of normal values to be set for each sensor and therefore allowing for "abnormal" conditions to be easily recognized. If an unsafe level of any kind is reached, the user is notified. This ensures a safe environment for operations, workers, and rescue personnel within the monitored structure. 


\subsection{System Verification}

Following the design of the network, additional testing, external sensor selection, calibration processes, and a small scale demonstration were performed in order to verify the system's features/capabilities and feasibility. The tests performed consisted of a simple alert test and then a topology test that encompassed the verification of all of the network's "Zigbee capabilities." Calibration processes were performed for various external sensors that were selected, and a small scale demonstration was staged.

\subsubsection{Alarm Testing}

As the battery life test was performed, alarm testing was also performed. One of the features of the software provided by Crossbow, Moteview, allows for the user to set alarm values for the various conditions being monitored. Using the data gathered from the environmental board, the alarm feature was tested using readings for temperature and light. Alarms can also be set for any other condition or sensor being monitored.

In order to test the lighting alarm, a node was placed in a dark area and an alarm value in lumens was set at a level to determine if a light had been turned on. When the light was turned on, the software reported the alarm threshold had been exceeded and an alarm was posted. For testing the temperature alarm, a 
second node was place outside with a lower limit value set for temperature. This node was also successful in triggering the alarm when the temperature fell below the set value.

\subsubsection{Topology}

In order to satisfy the system requirement of complete and redundant monitoring of the structure, the network must have many nodes and utilize multihop technology. Using a multi-hop technology allows for node to node communication rather than node to base station communication and adds scalability to the network in order to cover the entire area. Each node will also be able to transmit and receive data, allowing for the mesh network topology to be implemented which allows for sufficient redundancy to be achieved.

\subsubsection{Network Architecture}

The need for a self-healing network is the reason for using a mesh network topology. Mesh networks are multi-hopping systems in which router nodes are used. The figure below shows an example of a mesh network. 


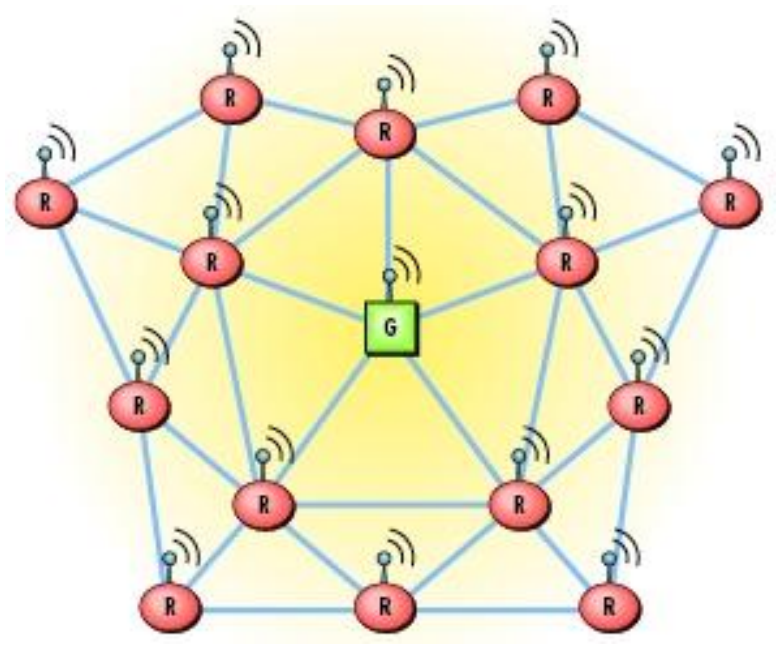

Figure 3.6: Mesh Network Topology.

(router nodes denoted by "R", gateway denoted by "G")[11]

The network self-configures itself such that these nodes pass data from one another to the base station in the optimal data path [11]. Using this network architecture, if a node fails, the network automatically reconfigures itself, or selfheals, and resends the data around the problem [11]. Since the mesh network utilizes multi-hop techniques, it provides scalability allowing for complete coverage of various structures. There are also drawbacks to using a mesh network. By using multi-hop techniques, there can be large delays because a data packet must travel through several nodes before reaching the base station or gateway [11]. Another drawback is due to the fact that the nodes must send and receive data. For this reason, the nodes consume more power. 
With the assumption that $\mathrm{AC}$ power will be available to the nodes in the designed network until interrupted, then power consumption is not a breaking point for selecting a mesh topology. The mesh network will provide high redundancy and high reliability, both of which are very important for the applications of this network.

\subsubsection{Topology/Network Capabilities}

The topology and Zigbee related features were then tested. The ground floor of the Engineering Sciences Building was used as a test site. The layout has stretches of hallway that provide line of sight as well as corners and parallel hallways to test the ability of the network to configure to the space. The figure below shows a basic, not to scale, layout of the Engineering Sciences Building ground floor with the placement of the base station and nodes throughout. 


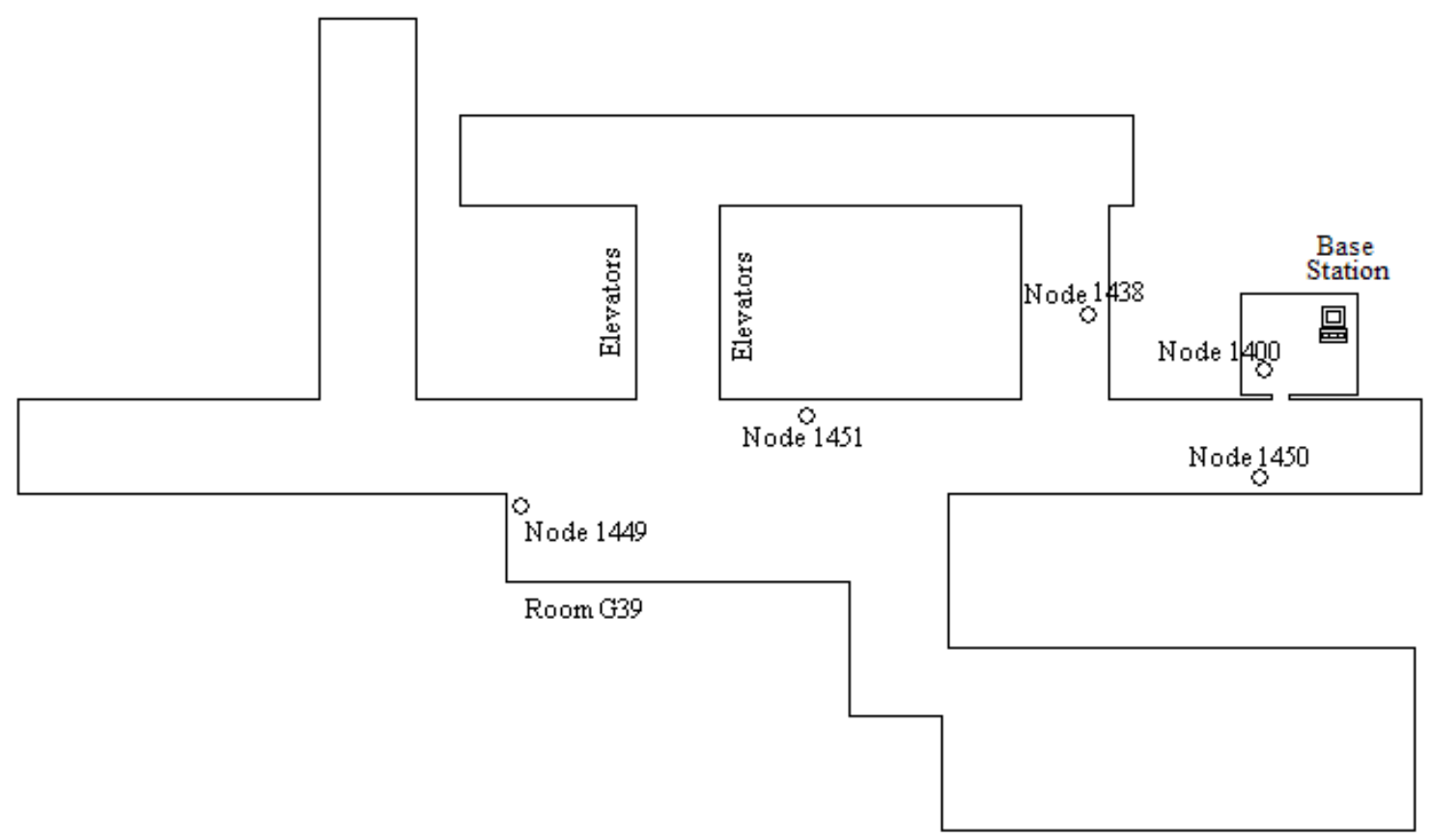

Figure 3.7: Layout of Topology Test on Ground Floor of Engineering Sciences Building

In order to test the various features, several tests were performed while monitoring the base station and recording relevant data. The nodes and base station were all placed in the appropriate positions and turned on. In order to verify the network's configuration, the topology of the network was monitored using Moteview. The paths of the node transmissions were shown and tracked throughout the entire topology test and are shown in the Results section.

Once the nodes were all detected and transmitting to the base station, the self-configuring feature of the network was confirmed. The second feature of the network that was in part tested with the data packet latency test was the multihop capabilities. The multi-hop capability was verified by placing node 1449 in a 
location where it relied on being transmitted through another node, node 1450 , to the base station. The topology results show this and the multi-hop ability of the network is again shown in the adaptive routing of the network that took place in the self-healing test.

Testing the ability of the network to self-heal was done by monitoring the paths of the data to ensure data was being passed through nodes 1450 and 1438. Node 1450 was then turned off, simulating its destruction or failure, and the data paths were rechecked to ensure that the data was routed around node 1450 to node 1438 and then through to the base station. Node 1450 was then

turned back on. The paths of the data were monitored again by using Moteview. As discussed further in the Results, the network adapted to node 1450 being reintroduced and found the best paths for the data travel.

\subsubsection{External Sensor Selection}

The Crossbow MDA320 board allows additional sensors to be connected to the wireless network and provide versatility. For the network designed, external sensors included a $\mathrm{CO}$ detector, an ultrasonic proximity sensor, a submersible pressure transducer, a string potentiometer, and a non-submersible pressure transducer. The ability to have a customizable bank of sensors allows this network to be applied to many possible scenarios, conditions, and locations. 


\subsubsection{Pepperl and Fuchs Ultrasonic Proximity Sensor}

One of the sensors selected to be connected to the DAQ board is an ultrasonic proximity sensor. The Pepperl and Fuchs flat pack ultrasonic proximity sensor, shown below, was selected to act as a device to detect water level in a possible flooding scenario.

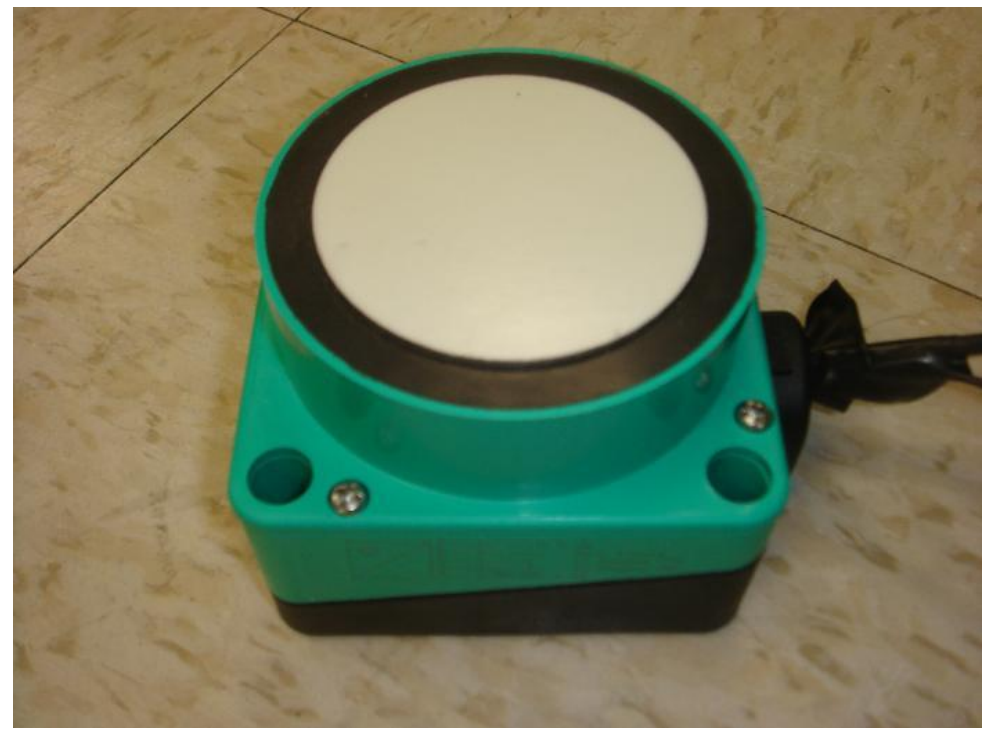

Figure 3.8: Pepperl and Fuchs Ultrasonic Proximity Sensor

The PF ultrasonic proximity sensor has a sensing range of $800 \mathrm{~mm}$ to $6 \mathrm{~m}$, providing an analog output of $4-20 \mathrm{~mA}$ or $2-10 \mathrm{~V}$. The analog voltage will be used and divided down to provide a $0.5-2.5 \mathrm{~V}$ range so it can be interfaced with the MDA 320 board. 


\subsubsection{American Sensor Technology Submersible Pressure Transducer}

An alternative method of measuring the water depth using the proximity sensor is a submersible pressure transducer. Shown below, the submersible pressure transducer acts to monitor water levels by monitoring the pressure head created by any given height of water. Since the proximity sensor and water level transducer will be monitoring the same thing, the better solution between the two will be selected. There is a possibility for the use of both water level methods depending on the application or the selected section of the structure.

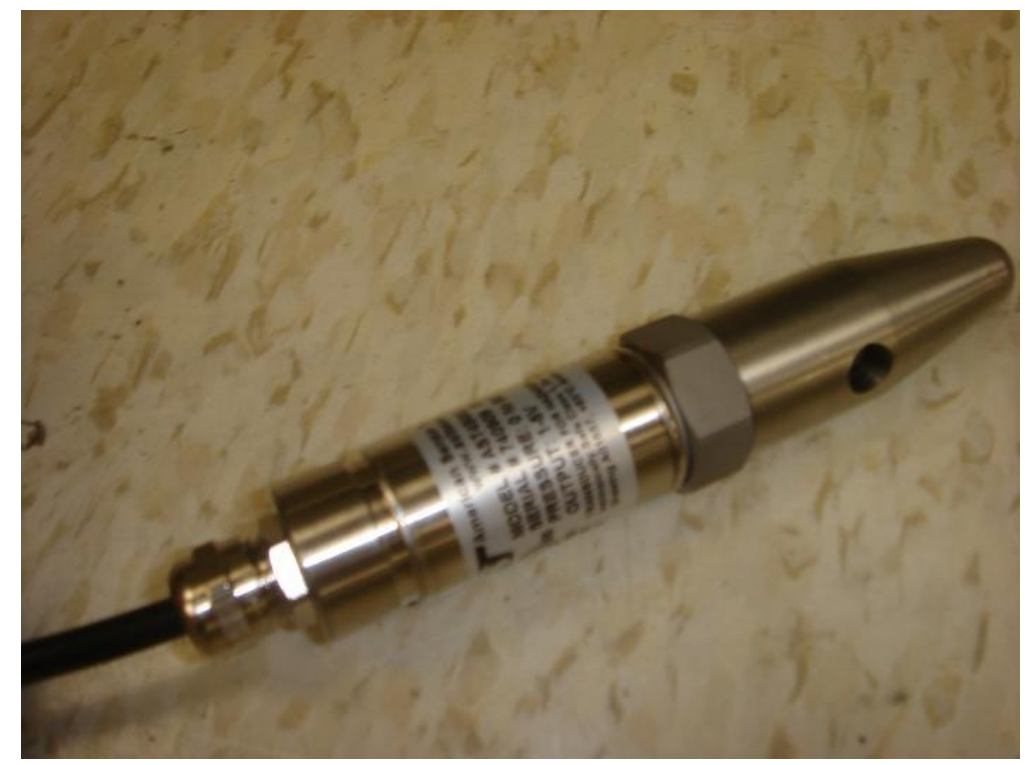

Figure 3.9: American Sensor Technology Submersible Pressure Transducer

The American Sensor Technology submersible pressure transducer, shown above, has a range of $0-50$ psi and will be used for demonstration 
purposes as well as for actual implementation. The selected sensor has an output of $1-5 \mathrm{~V}$ which was divided down to $0.5-2.5 \mathrm{~V}$ and then interfaced through the MDA 320 DAQ board.

\subsubsection{CO Detector}

For demonstration purposes, a household $\mathrm{CO}$ detector was purchased and disassembled. After disassembling the detector, a signal lead and ground lead were attached to the appropriate locations and then connected to a digital input on the MDA320 board. A diagram showing how the CO detector and ultrasonic proximity sensor were wired to the MDA 320 board is shown below in Figure 3.10.

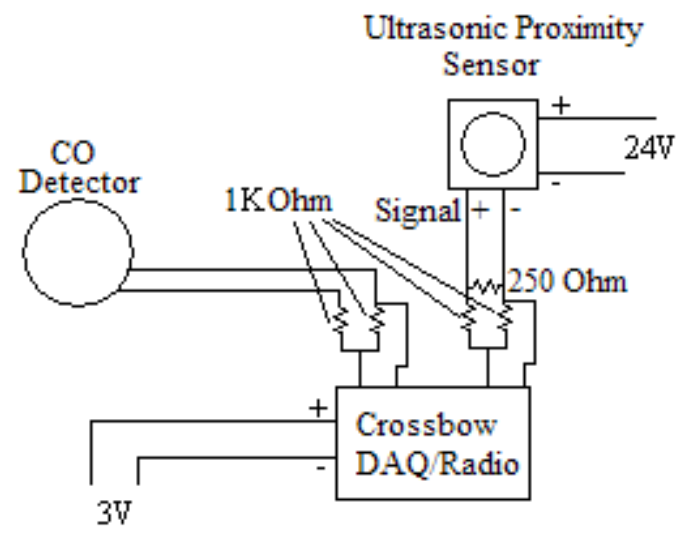

Figure 3.10: Wiring Schematic CO Detector and Ultrasonic Proximity Sensor for a Single Node 
For actual implementation of a CO detector, an industrial sensor should be acquired. However, it was more feasible to use a simple CO detector to illustrate the potential uses rather than an industrial instrument. Industrial $\mathrm{CO}$ detectors would be more robust and reliable as well as provide an analog output rather than a simple digital output. This would be ideal, but when they were researched, industrial CO detectors were $\$ 200$ + as opposed to the cost of $\$ 12$ to use a household detector and show the sensor as an option for implementation.

\subsubsection{Sensor Accuracy}

With the use of sensors and an analog to digital converters, accuracy becomes an issue to consider.

The MDA 320 DAQ board has a resolution of 16 bits and input voltage of 0 -2.5V gives a quantization error of approximately $\pm 19 \mu \mathrm{V}$. This is a negligible value when it is compared to the error or resolution of the selected sensors as shown in their respective descriptions.

The quoted accuracy of the ultrasonic proximity sensor is $\pm 1.82 \mathrm{~mm}$ over the full scale. This translates into an error or resolution of $\pm 860 \mu \mathrm{V}$. When compared to the calculated quantization error, the resolution of the sensor is the prominent source of error in the measurement output of the ultrasonic proximity sensor.

As stated in the specification, the accuracy of the submersible pressure transducer is $\pm 0.25 \%$. The worst case error is present at the full scale value of 
50 psi. This translates into a resolution or possible error of \pm 0.125 psi or \pm 6.25 $\mathrm{mV}$ which is much larger than the possible error that would result from the quantization error of the MDA 320 board. This shows again that the error induced by the sensor is the only error that needs to be considered for the output of the submersible pressure transducer.

Along with the consideration of accuracy at the selection and implementation, consistent, reliable performance is also required throughout the anticipated life of the sensor. One possible cause for a decrease in consistency or reliability is transducer drift. Transducer drift comes from sources such as changes in temperature, humidity, and power supplied.

The intended application of this wireless sensor network is to monitor underground structures. In underground structures, the stated sources do not present an issue due to the relatively steady state environment within the structure.

\subsubsection{Demonstration Hardware}

For the sole purpose of demonstrating the network in small scale with simulated flooding conditions, two additional sensors were added to the network. The two additional sensors that were added include a string potentiometer and a non-submersible pressure transducer with an LED display. The Unimeasure string potentiometer or extensometer that was selected is shown below. 


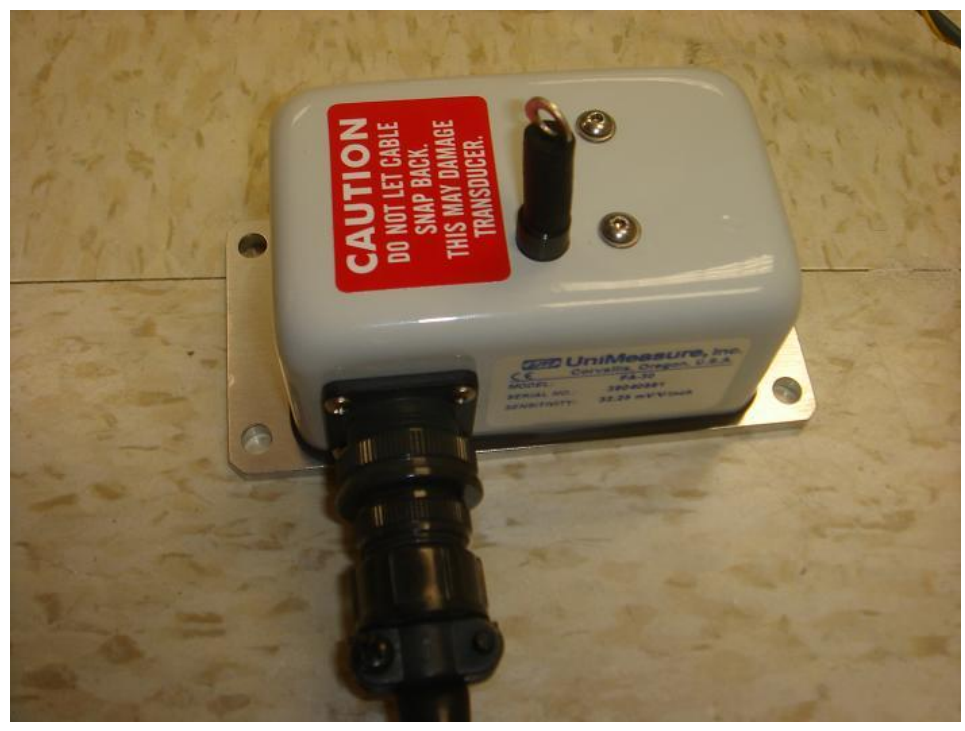

Figure 3.11: Unimeasure String Potentiometer

The Unimeasure string potentiometer has an output voltage equal to that of the input voltage. It was provided with 3 volts via two $\mathrm{D}$ cell batteries and a battery charger. The detectable extension is $0-30$ inches which outputs $0-3 \mathrm{~V}$ which was then divided down to $0-1.5 \mathrm{~V}$ to interface with the MDA 320 board. 


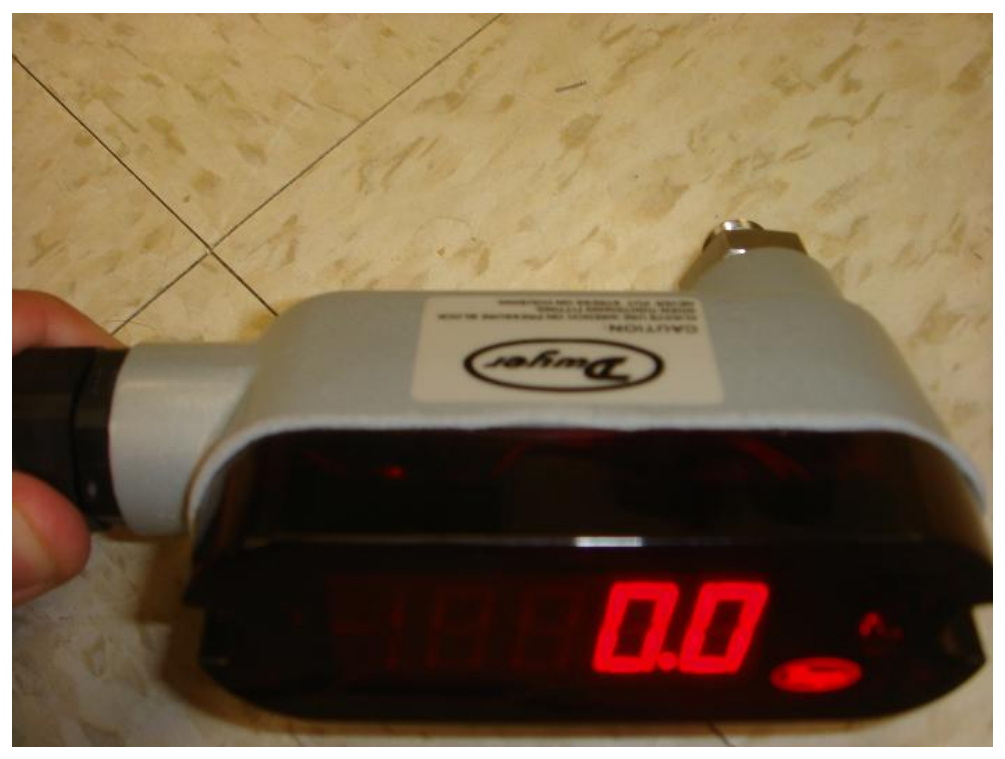

Figure 3.12: Dwyer Non-submersible Pressure Transducer with LED Display

Figure 3.12 shows the second of two demonstration sensors that were selected. This sensor was the Dwyer non-submersible pressure transducer with LED display. This sensor has a range of $0-50 p s i$, was supplied with $24 \mathrm{~V}$, and output $0-5 \mathrm{~V}$. The output was again divided down and interfaced with the Crossbow DAQ board.

Two nodes were selected and setup with MDA 320 boards and sensors were interfaced with them. The additional sensors were the string potentiometer, submersible pressure transducers, and non-submersible pressure transducers. A diagram showing how the additional sensors were interfaced with the Crossbow equipment is shown below. 


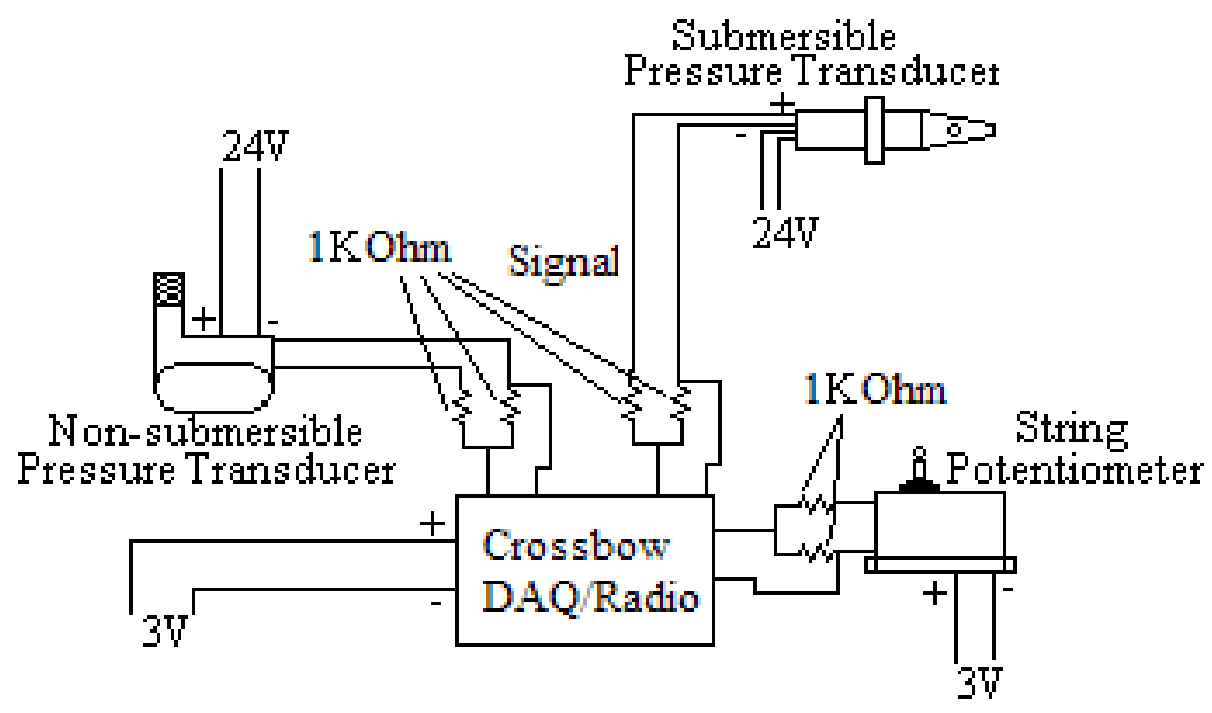

Figure 3.13: Wiring Schematic with Submersible and Non-Submersible Pressure Transducers, and String Potentiometer Sensor for a Single Node

\subsubsection{Power Supply for External Sensors}

Addition of a customizable bank of sensors provides versatility to the network; however, some sensors require higher supply voltages than the radio modules and sensor boards as illustrated in Figures 3.10 and 3.13. This led to the design of power supply boxes. The boxes are NEMA type 4 enclosures containing batteries, chargers, and terminal strips. The layout of the boxes is shown in Figure 3.14, and pictures of the construction will follow in the Results section. 


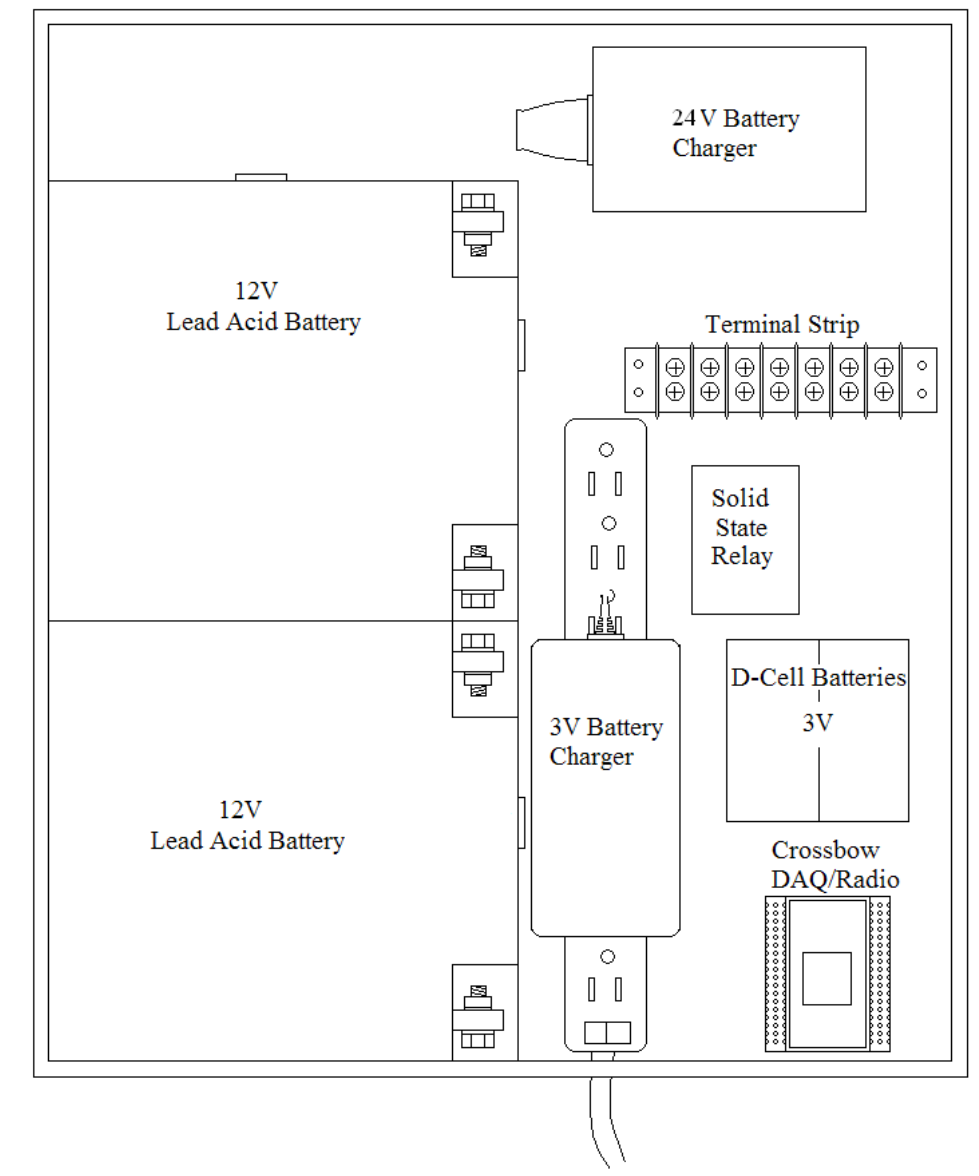

Figure 3.14: Layout of Power Supply Boxes

Housing the batteries, chargers, wiring, and Crossbow components in these boxes makes them less susceptible to things like dust, dampness, vermin or insects, and shock. The power boxes will be supplied with 110 VAC that will continuously charge backup batteries for when the AC power is lost. This allows for the sensors and radio nodes to run for approximately 100 to 150 hours following the stoppage of AC power. Batteries can be added or the current batteries can be used to supply power to control supplementary systems such as the aforementioned emergency devices or other various sensors. 


\subsubsection{Construction of Power Supply Boxes}

Once the boxes were designed and laid out, the power boxes were constructed. This started by wiring two lead-acid batteries, supplying 12 volts each, in series to be capable of powering the ultrasonic proximity sensor and the two pressure transducers. Two D-cell batteries were added to power the radio module and the environmental boards for longer than the standard AA cell batteries. All of the batteries were wired to terminal strips allowing for access to the power. Battery chargers were attached to each set of batteries via the terminal strip and were connected to AC power. The batteries continuously charge until the AC power is interrupted. From the terminal strips, the sensors and Crossbow hardware were connected. The ultrasonic proximity sensor is housed in a smaller enclosure allowing it to be placed in a different location than the power boxes, such as the roof of a mine or tunnel. The IRIS mote with the attached MDA 320 board and MTS 400 environmental board was connected to the two $\mathrm{D}$ cell batteries. The proximity sensor, submersible pressure transducer, non-submersible pressure transducer, and $\mathrm{CO}$ detector were then connected to the MDA320 DAQ boards using analog and digital inputs. Figure 4.4 shows a wiring schematic of the power supply boxes and attached sensors. 


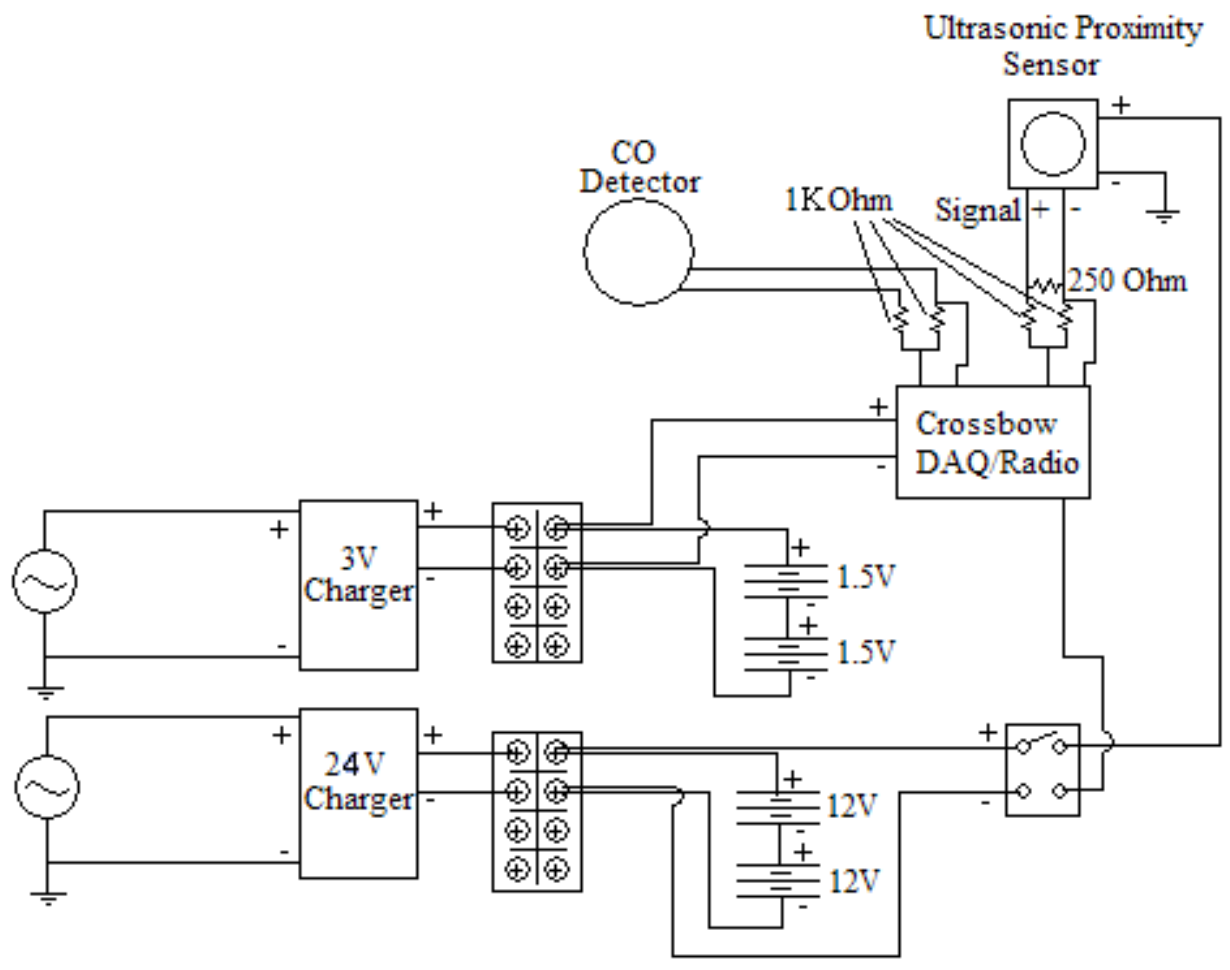

Figure 3.15: Wiring Schematic of Power Supply Boxes with CO Detector and Ultrasonic Proximity Sensor

Figure 3.15 shows the power boxes connected to the Crossbow $\mathrm{DAQ} /$ Radio modem as well as the CO detector and the ultrasonic proximity sensor. As shown in Figure 3.13, the voltages provided by the power box also satisfy the submersible and non-submersible pressure transducers as well as the string potentiometer. This means that any of the sensors can be added or removed for various configurations. Figure 3.16 shows another possible configuration using both types of pressure transducers and the string potentiometer. 


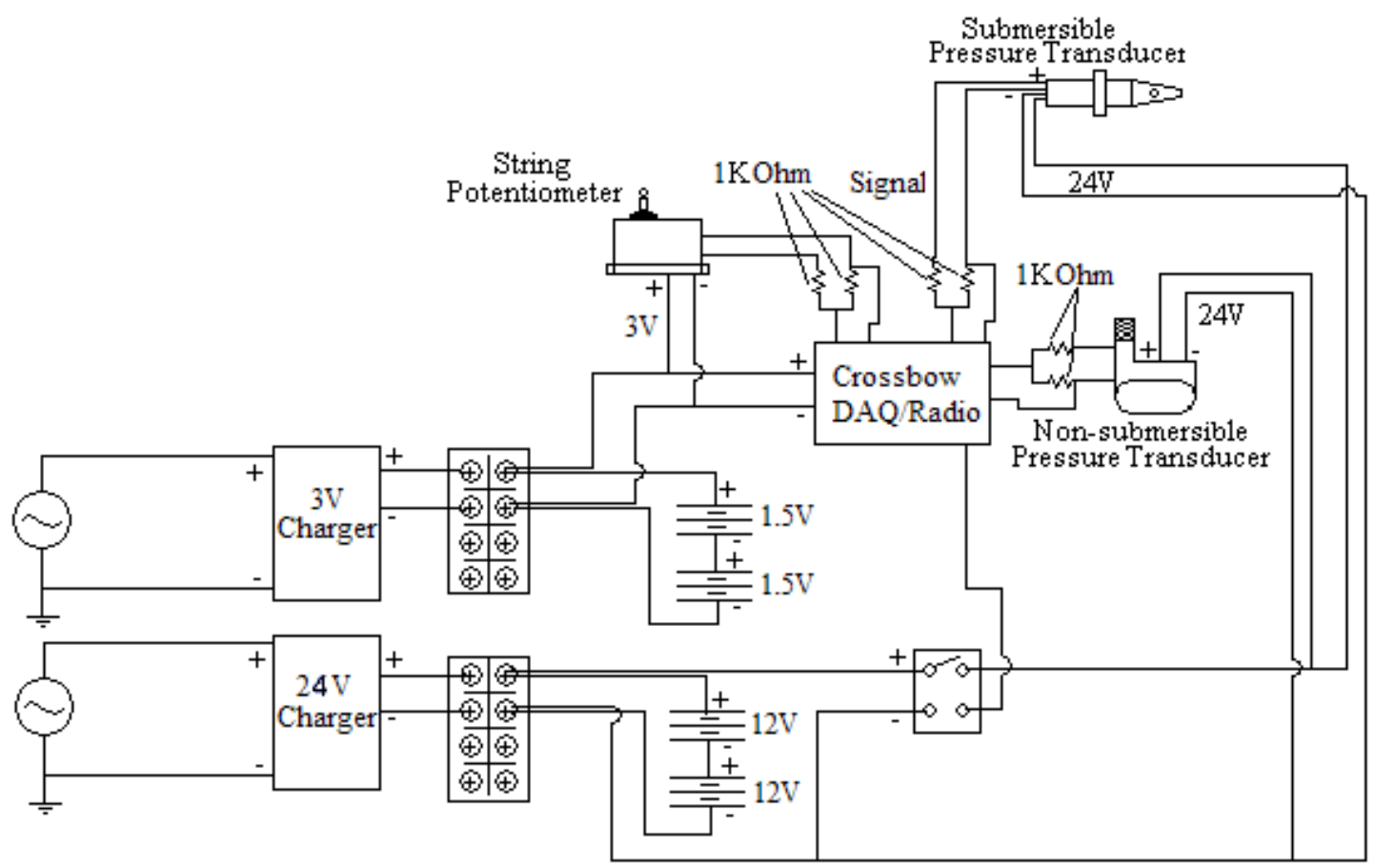

Figure 3.16: Wiring Schematic of Power Supply Boxes with Pressure Transducers and String Potentiometer

\subsubsection{Verification of External Sensor Calibration}

Following the selection of all of the external sensors to be added to the network, verification of the factory calibration was done using each selected sensor and the Crossbow hardware. This ensured the output to the base station was accurate to the actual measurement being monitored. 


\subsubsection{Ultrasonic Proximity Sensor}

Verification of the calibration of the proximity sensor consisted of setting the range to full scale and moving a large piece of plywood, simulating water level, to pre-measured distances perpendicular from the sensor. A voltage reading at each distance was then taken and the MDA320 board was programmed to interpret the voltage output as a corresponding distance.

\subsubsection{Submersible Pressure Transducer}

The submersible pressure transducer calibration was then verified using given specifications for the sensor and the large tank of water at the West Virginia University Hangar. The submersible pressure transducer that was used produced an output voltage of $1-5 \mathrm{~V}$ which was then divided down to $0.5-2.5 \mathrm{~V}$ over the range of $0-50 p s i$. This data was used to generate a calibration line which gave the equation to convert the voltage to a pressure output. Using equations of fluid dynamics, this pressure was then converted into a height or in this case a depth in feet that was again programmed into Moteview to output a depth rather than a voltage. The pressure transducer was then lowered into the tank of water while the depth reading was monitored on the computer. The depth reading was accurate for the given depths of water and verified the proper calibration of the sensor. 


\subsubsection{Non-Submersible Pressure Transducer}

The non-submersible pressure transducer provided an output of $0-5 \mathrm{~V}$, which was divided down to $0-2.5 \mathrm{~V}$, over the range of 0 to $50 \mathrm{psi}$. This data was used to generate a calibration curve that produced a pressure in psi rather than a voltage. The non-submersible pressure transducer was tested and calibrated using a pressurized air supply from a compressor. An air hose was run from a compressor, through a regulator with an analog pressure gauge to the Dwyer

pressure transducer. The air pressure was then adjusted using the regulator to different levels. The analog gauge and LED display of the Dwyer gauge were compared to verify a proper reading. The information was then monitored through the base station and verified as accurate.

\subsubsection{String Potentiometer}

The string pot was connected to the 3V power supply and the MDA 320 board and then calibrated in a similar fashion as the ultrasonic proximity sensor. The string was pulled along a ruler to various lengths. The voltage at each length was recorded on the base station and used to verify the calibration information that was provided with the sensor. 


\subsubsection{CO Detector}

The $\mathrm{CO}$ detector was also tested, but by using the "Test" button on the sensor to simulate an alarm rather than producing $\mathrm{CO}$ to trip the alarm. This produced a false alarm that allowed for a reading to be output to the base station via a digital input on the MDA 320 board.

\subsubsection{Demonstration}

Once the entire system has been tested and calibrated, a small scale demonstration was performed to show the overall functionality and specific features of the network. The West Virginia University Hangar was used as the site for the demonstration. The network was set up with the external sensors for the demonstration were attached to the MDA 320 DAQ board in a position to monitor the required parameters for the demonstration of a mechanical plug in a small scale tunnel with various flooding scenarios. The sensors for the demonstration consisted of pressure transducers, both submersible and nonsubmersible and the string potentiometer.

The demonstration itself was performed in one day, but testing and setup were performed several times to make sure the network and sensors were working properly. In the demonstration, several flooding scenarios were produced and pertinent data was recorded from the pressure sensors and the 
string pot. The non-submersible pressure transducers were used to monitor the internal pressure of the inflatable plug by way of a drainage tube connected to the plug and the pressure applied to the exterior of the plug. The submersible pressure transducer was used to monitor the water depth on the open side of the small scale tunnel. The string pot was used to monitor the displacement of the plug in the longitudinal direction of the tunnel and the deflection of the face of the plug that was experiencing the most pressure. Figure 3.17 shows a representation of the layout of the demonstration with the placement of the sensors, how the plug will be filled, and how the tunnel will be flooded. This figure is not to scale.

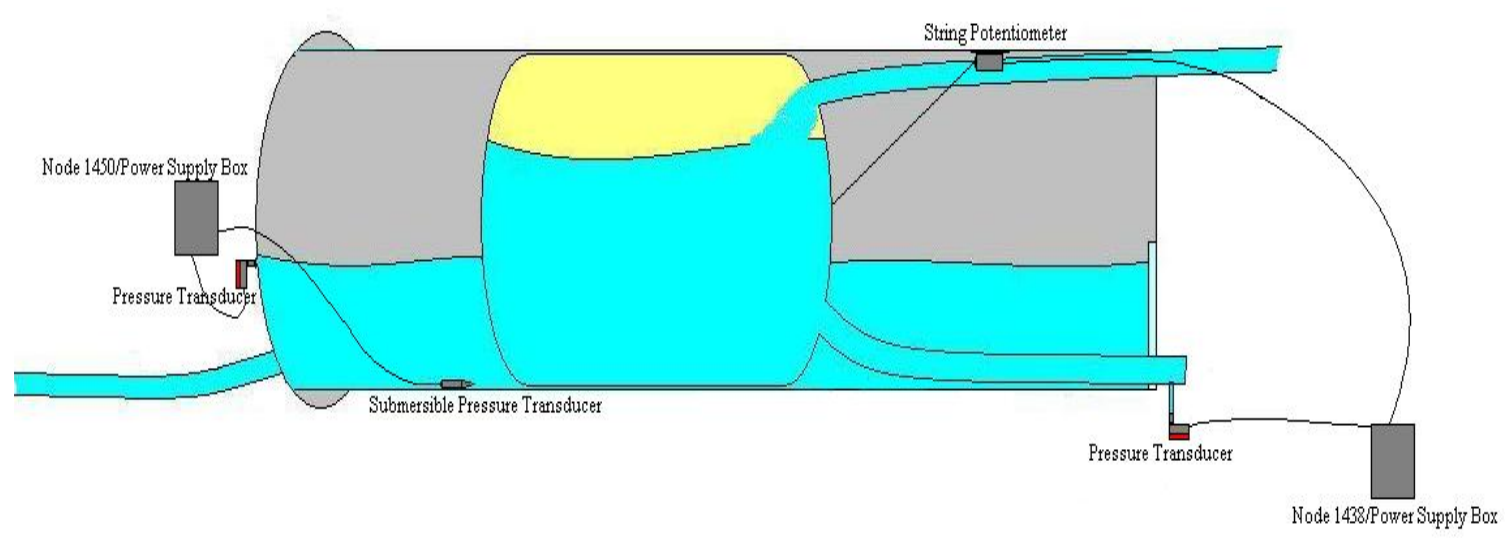

Figure 3.17: Layout of Small-Scale Demonstration

As with all of the testing that was performed, results and data were collected and displayed throughout the demonstration and reviewed following the demonstration. 


\section{Chapter 4: Results}

\subsection{Construction of Power Supply Boxes}

Upon designing the power supply boxes and providing schematic representation, physical construction of the boxes took place. To start, a plywood mounting board was installed into a NEMA Type 4 enclosure. Next, two $12 \mathrm{~V}$ lead acid batteries were mounted in the box actively held by metal brackets as shown below and passively held in place by foam board as shown in Figure 4.1. The surge bar was then installed to supply power to the battery chargers. The 24V battery charger was then mounted and plugged into the surge bar and connected to the terminal strip. A solid state relay and D-cell battery housing were installed next. The solid state relay can be used for limiting battery consumption in future applications by utilizing the digital outputs of the Crossbow board. The next step was the addition of the $3 \mathrm{~V}$ battery charger. The charger, like the $24 \mathrm{~V}$ charger, was plugged into the surge bar and attached to the terminal strip. Finally, the Crossbow module was added to the enclosure and all the components were wired for operation. Power was then run to the Crossbow module. As mentioned, the $12 \mathrm{~V}$ batteries are passively held in place by the blue foam board pictured when the lid is closed. 


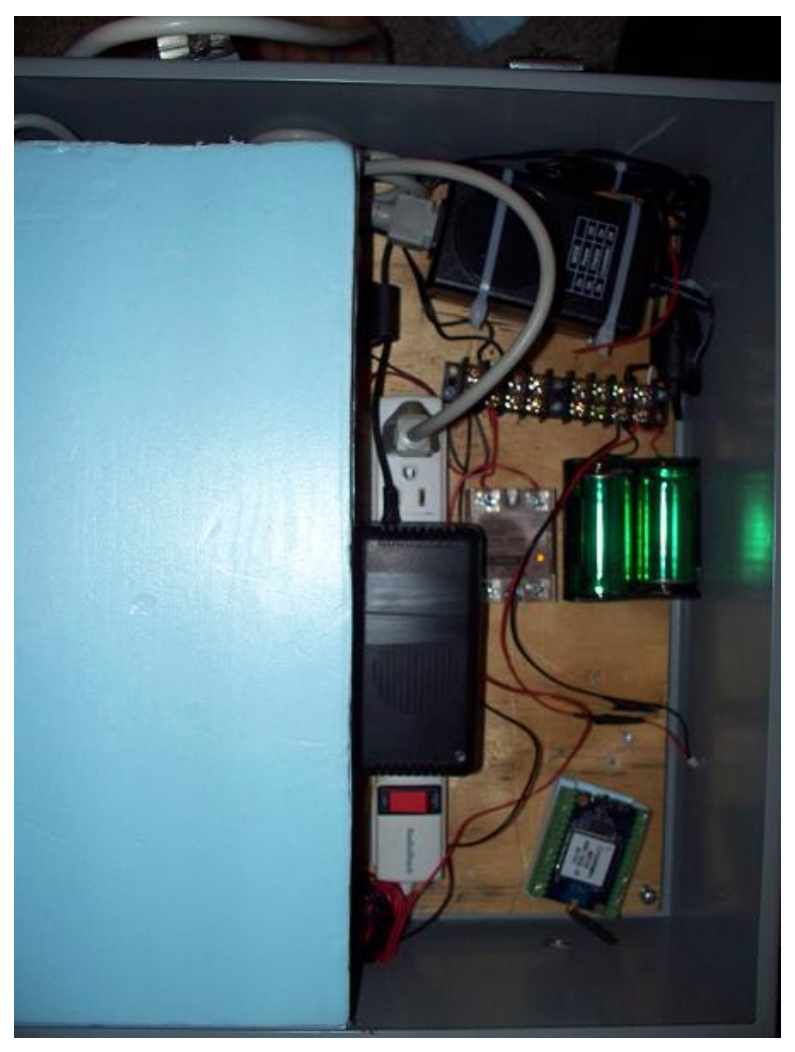

Figure 4.1: Batteries, Chargers, and Crossbow Module Completely Installed

The submersible and non-submersible pressure transducers were connected to $24 \mathrm{~V}$ power supplied by the two $12 \mathrm{~V}$ lead acid batteries in series and then connected to the Crossbow module. At this point, the power supply boxes were completed as designed and ready to be implemented into testing and calibration. 


\subsection{Network Design Test Data}

Data from the various tests that were performed for design purposes was recorded and analyzed as shown in the following section.

\subsubsection{Range}

The range test was intended to aid in the design of the network. By determining the effectiveness of the nodes at full scale range and intermediate distances, levels of redundancy can be established. Table 4.1 shows the data from five nodes that was recorded and reduced from the procedure described in the Technical Approach section. Figure 4.2 shows the data again, in graphical form.

Table 4.1: Percentage of Dropped Packets for Five Nodes at Various Distances

\begin{tabular}{|r|c|r|r|r|r|}
\hline & \multicolumn{5}{|c|}{ Dropped Packets (\%) } \\
\hline Node ID & $\mathbf{3 0 0 m}$ & \multicolumn{1}{|c|}{$\mathbf{3 5 0}$} & $\mathbf{4 0 0 m}$ & $\mathbf{4 5 0 m}$ & 500m \\
\hline $\mathbf{1 4 4 5}$ & 1.64 & 5.48 & 11.65 & 14.84 & 27.52 \\
\hline $\mathbf{1 4 4 9}$ & 4.79 & 8.33 & 12.64 & 5.26 & 4.01 \\
\hline $\mathbf{1 4 5 0}$ & 3.72 & 20.62 & 7.86 & 13.78 & 2.49 \\
\hline $\mathbf{1 4 5 1}$ & 0.45 & 2.62 & 13.68 & 6.57 & 21.57 \\
\hline $\mathbf{2 2 0 6}$ & 4.48 & 9.97 & 5.24 & 8.65 & 6.36 \\
\hline
\end{tabular}




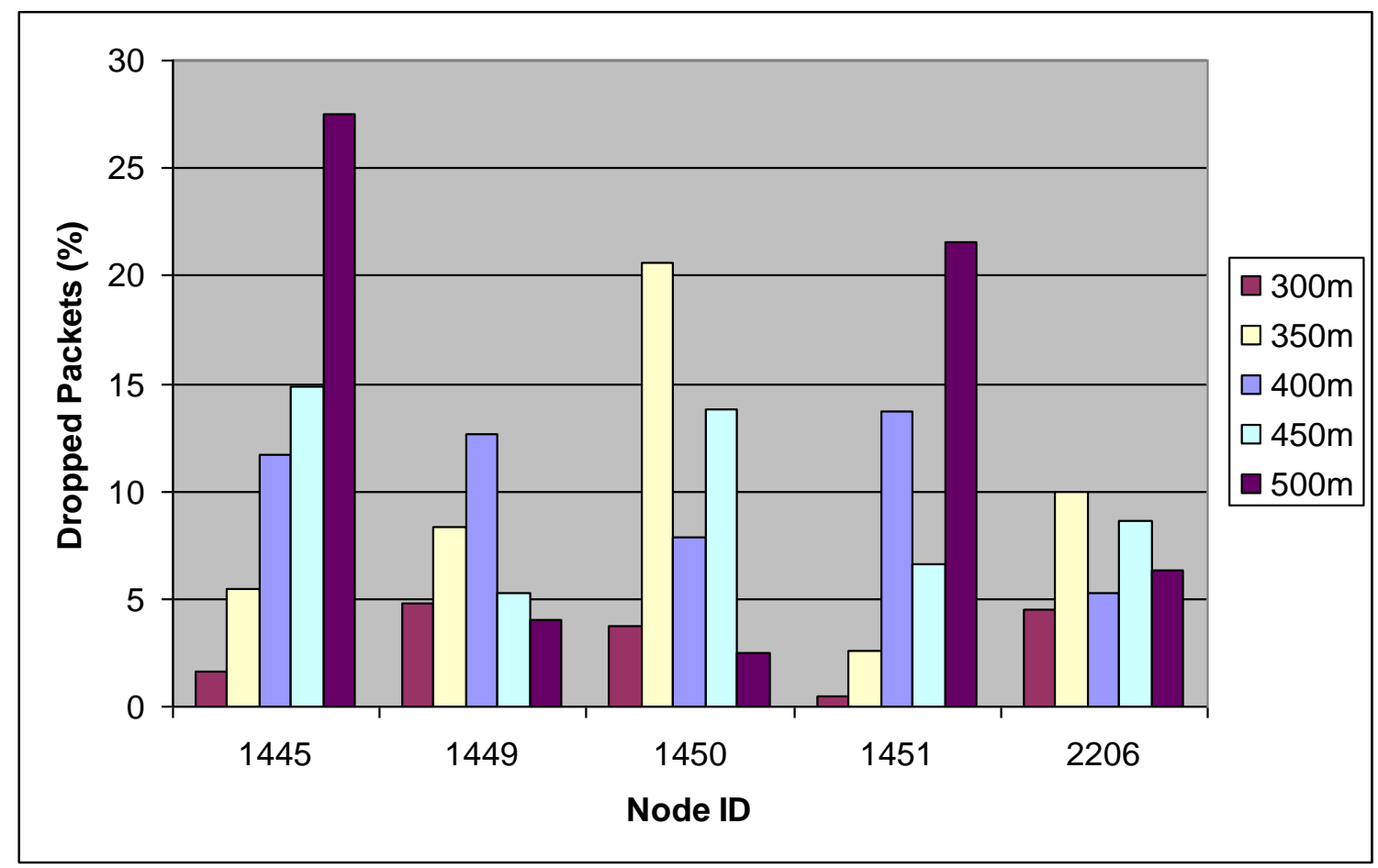

Figure 4.2: Percentage of Dropped Packets for Five Nodes at Various Distances

This data shows no direct correlation between the distance and the percentage of dropped packets meaning that regardless of the distance at which the node was located in the test, the data transmission was equally reliable. Using the given software, it was not possible to measure the data in a quantitative manner to gain a clearer understanding of the relationship between distance and reliability. The data shows that the network operated reliably at $500 \mathrm{~m}$ which verifies the quoted range of the hardware used. 


\subsubsection{Data Latency}

In the described data latency test, three trials were performed. The data in Table 4.2 shows each trial and the time in seconds for the tested nodes to reestablish communication with the base station. Figure 4.3 shows the results again, in graphical form.

Table 4.2: Data Latency Results

\begin{tabular}{|c|r|r|r|r|}
\hline Trial & $\mathbf{1}$ & \multicolumn{1}{|c|}{$\mathbf{2}$} & \multicolumn{1}{|c|}{$\mathbf{3}$} & \multicolumn{1}{c|}{ Average } \\
\hline $\begin{array}{c}\text { Time Until 1st Node } \\
\text { Reestablished }\end{array}$ & 40.5 & 40 & 37.8 & 39.43 \\
\hline $\begin{array}{c}\text { Time Until 2nd Node } \\
\text { Reestablished }\end{array}$ & 68.3 & 69.3 & 44.6 & 60.73 \\
\hline
\end{tabular}

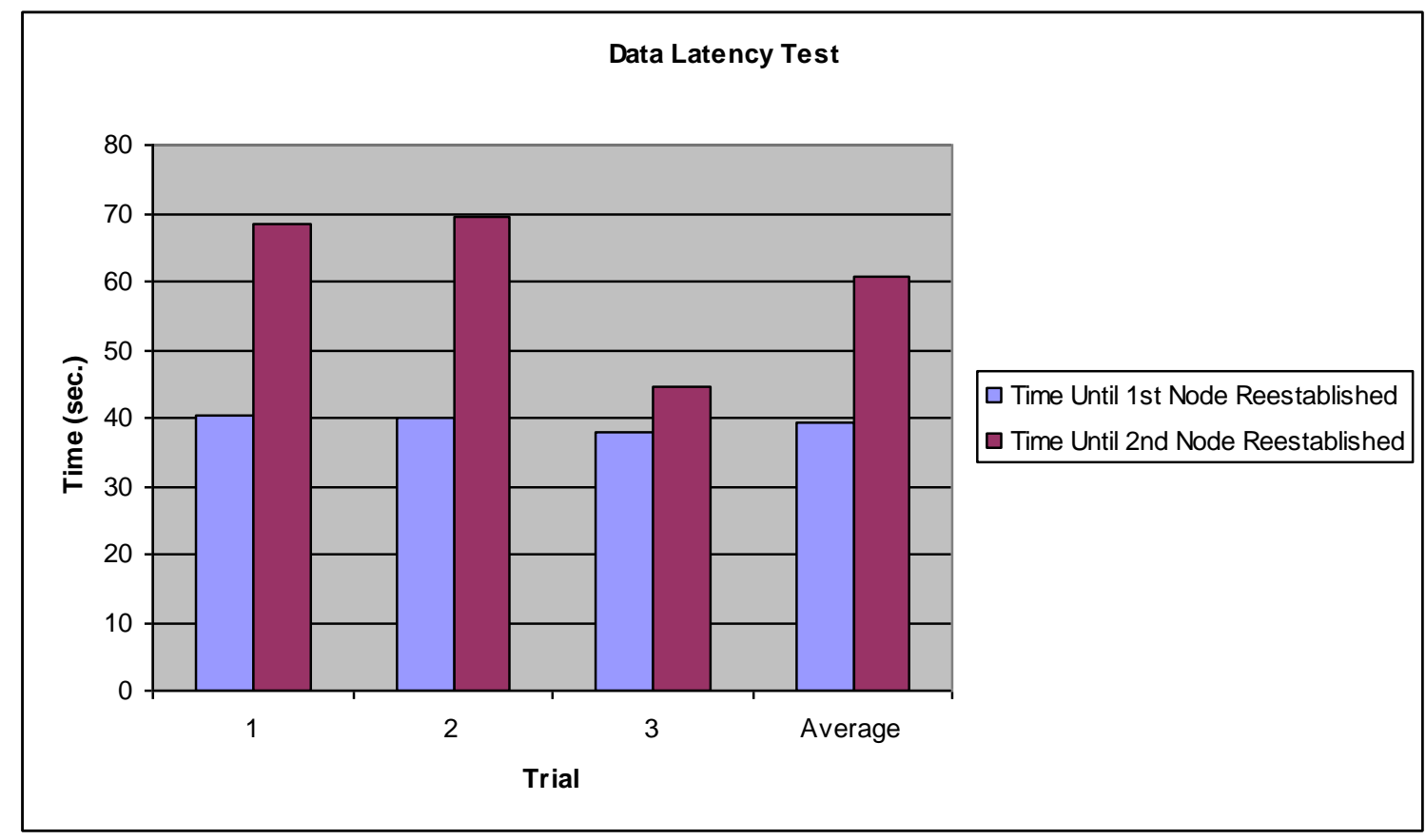

Figure 4.3: Data Latency Test Results 
The data from the test shows a reacquisition time of 40 seconds for the first node and 60 seconds for the second node. This is fast enough that sufficient time will still be available for proper assessment of the situation and to make a decision regarding actions to be taken; however, this does not allow for localization of something as fast moving as a blast from an explosion or train pressure wave.

\subsection{System Verification Data}

The following section describes brief procedural steps that were taken to verify the system capabilities as mentioned in the Technical Approach and groups them with the corresponding data that was recorded.

\subsubsection{Topology}

The topology test was performed as the first portion of the system verification of the overall network. Figures 4.4 through 4.10 show various data paths that the network used to route data while using the same topology. The placement of the nodes in the figures is not to scale but the general topology of the network is represented. Figure 4.4 shows the initial setup of the network after all of the nodes have been turned on and allowed to route the data through the best possible path. As stated in the Technical Approach, this topology verifies the network's ability to self-configure. Figure 4.4 also shows the 
network's multi-hop capabilities. This is illustrated by the fact that 1451 transmits to the base station through 1450 . These two features of the network are also shown in Figures 4.5 through 4.10.

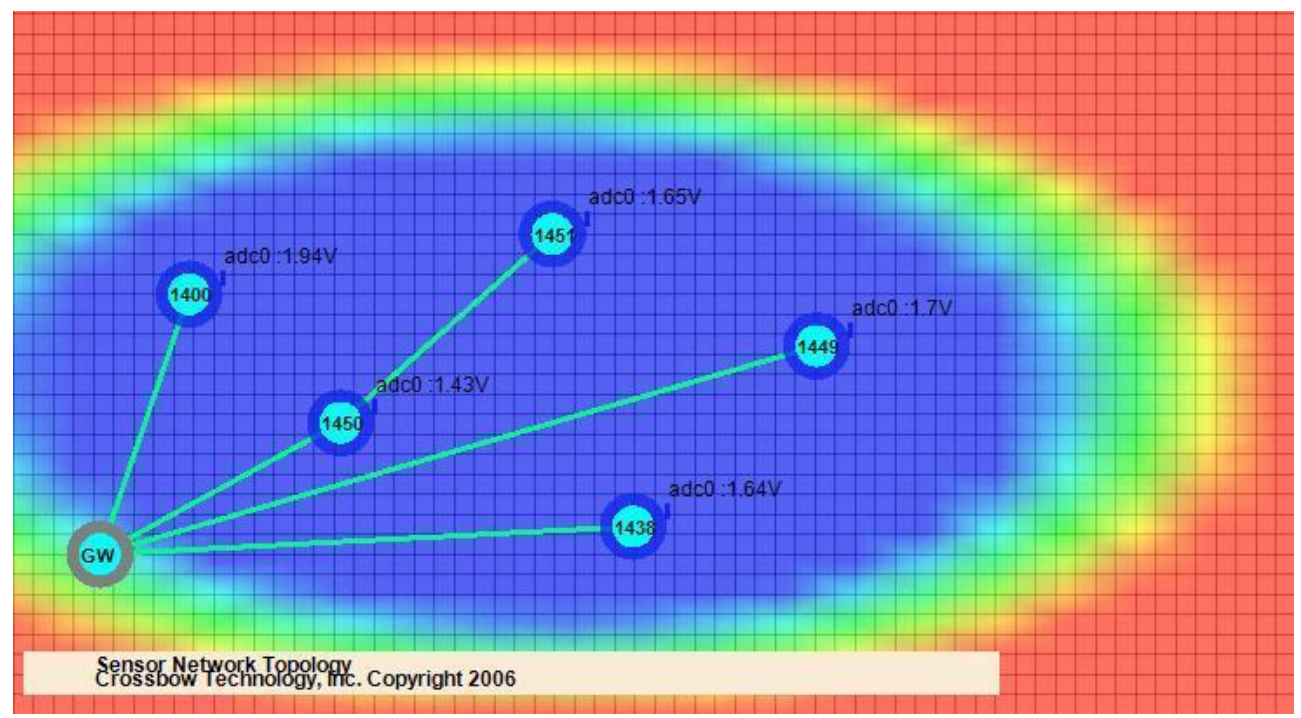

Figure 4.4: Topology 1: Initial Setup with Nodes Transmitting on Best Available Data Path

The next portion of the topology test was to demonstrate the network's ability to self-heal. In Figure 4.5, node 1450 has been turned off, simulating its destruction. As shown in the figure, 1451 rerouted itself through 1438. This topology change required no input from the user. 


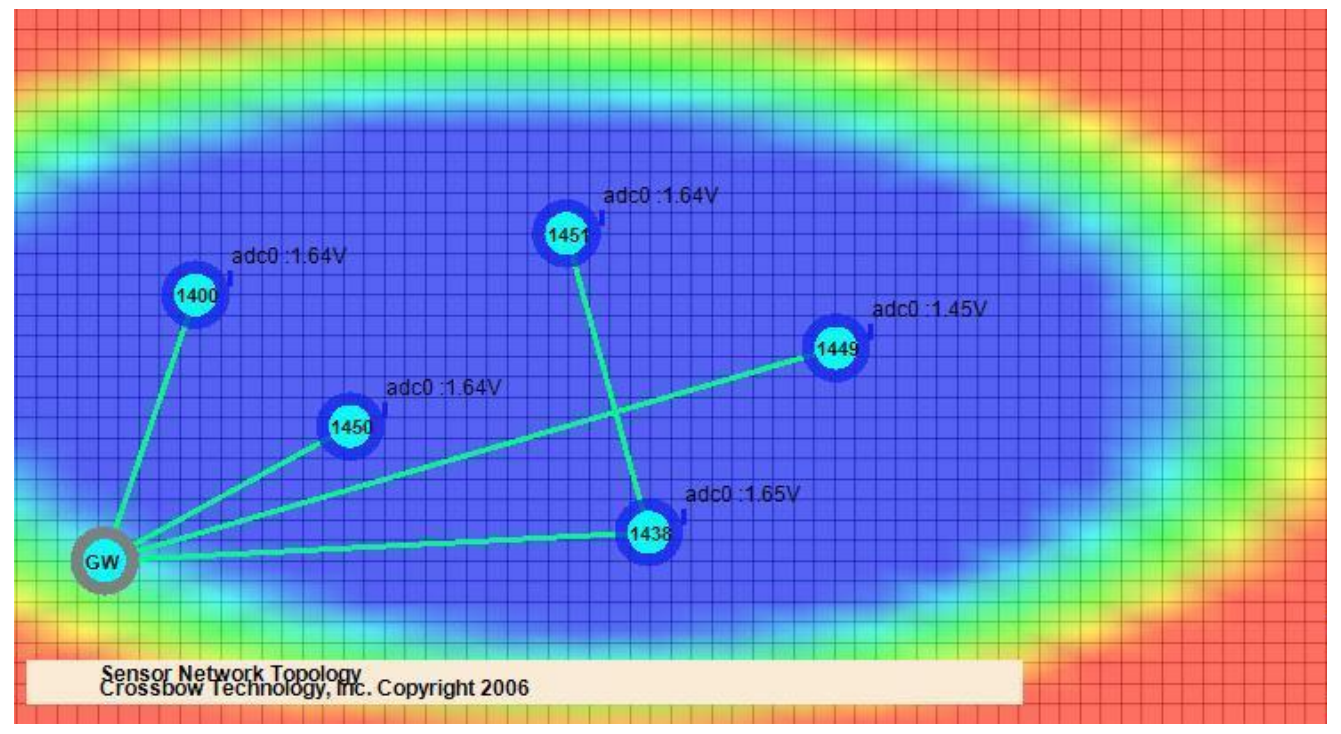

Figure 4.5: Topology 2: Simulated Node 1450's Destruction. Network Self-Healed by Rerouting Node 1451 through Node 1438

Topology 3, as shown in Figure 4.6, was generated while monitoring the network configuration. After node 1450 was turned off for 20 to 30 seconds, node 1449 selected another data path and began transmitting through node 1451 and 1438. This demonstrated an adaptive rerouting capability of the network due to the fact that it is constantly looking for the best possible data path for the nodes. 


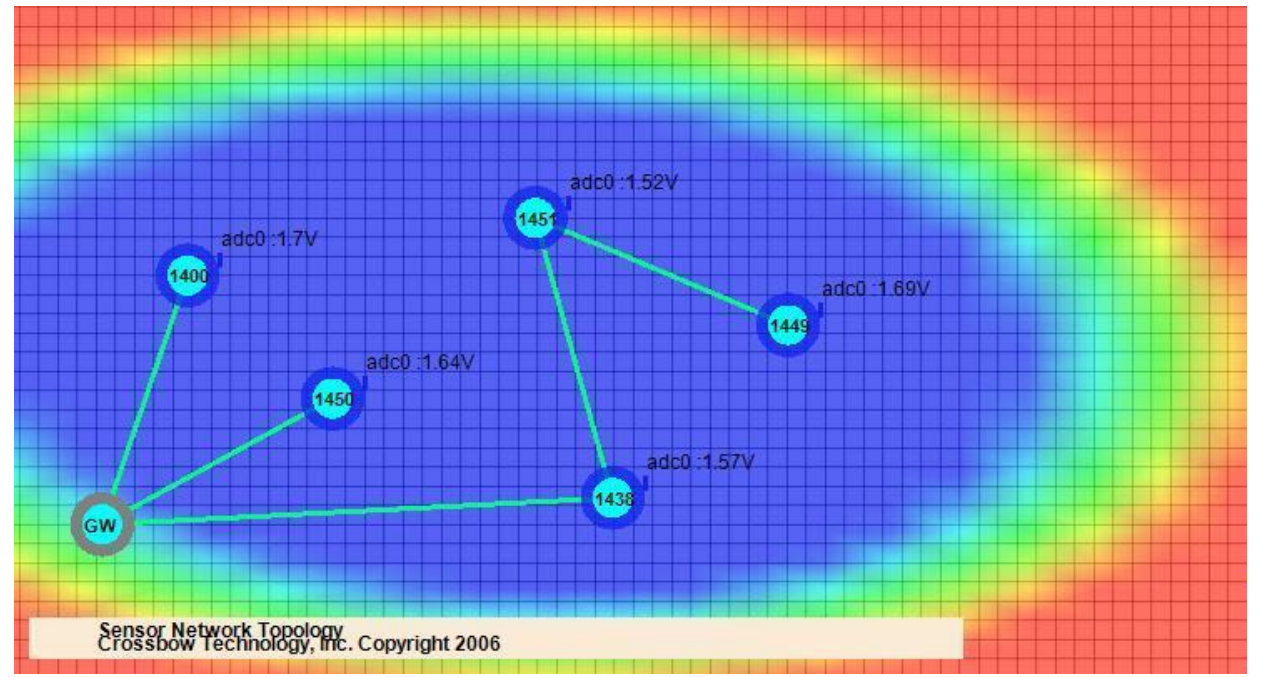

Figure 4.6: Topology 3: Node 1449 Selected a Better Data Path and Adaptively Rerouted through Node 1451.

Node 1450 was turned back on in Topology 4. Figure 4.7 shows node 1450 initially selecting a data path through node 1400 .

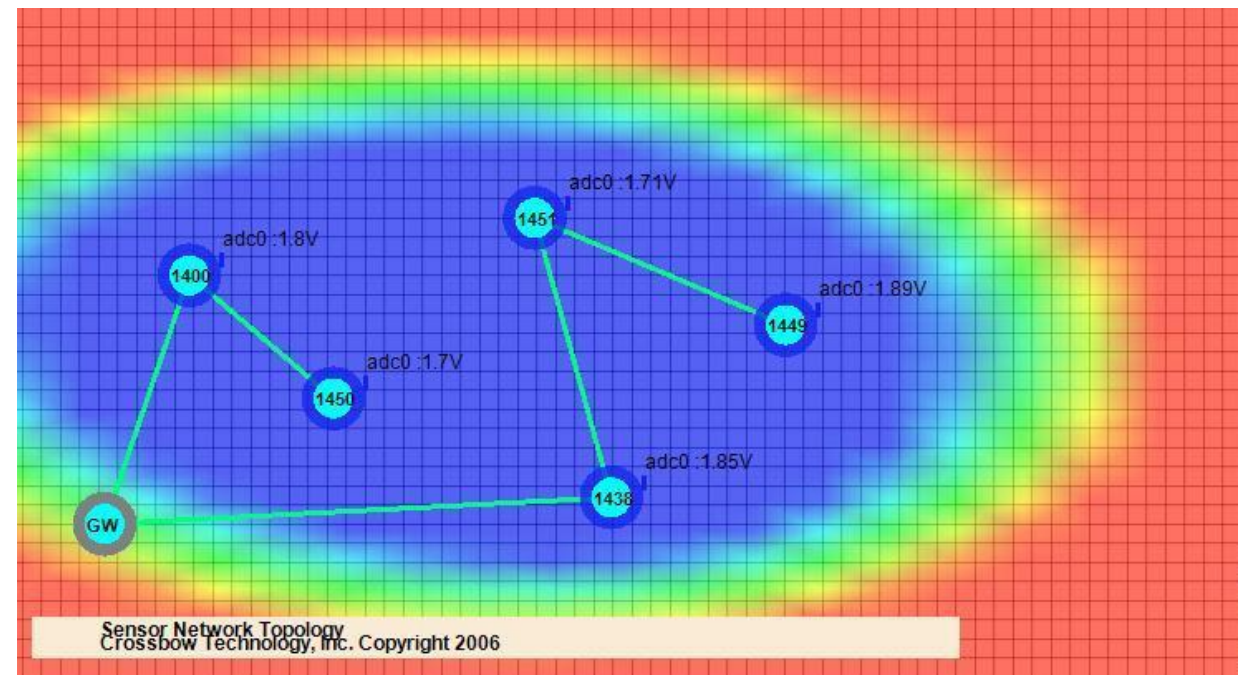

Figure 4.7: Topology 4: Node 1450 Turned On and Routes Itself through Node 1400 
The network was monitored following the reintroduction of node 1450 .

After a brief period of time, node 1450 found direct communication with the base station and selected a new data path as shown in Figure 4.8.

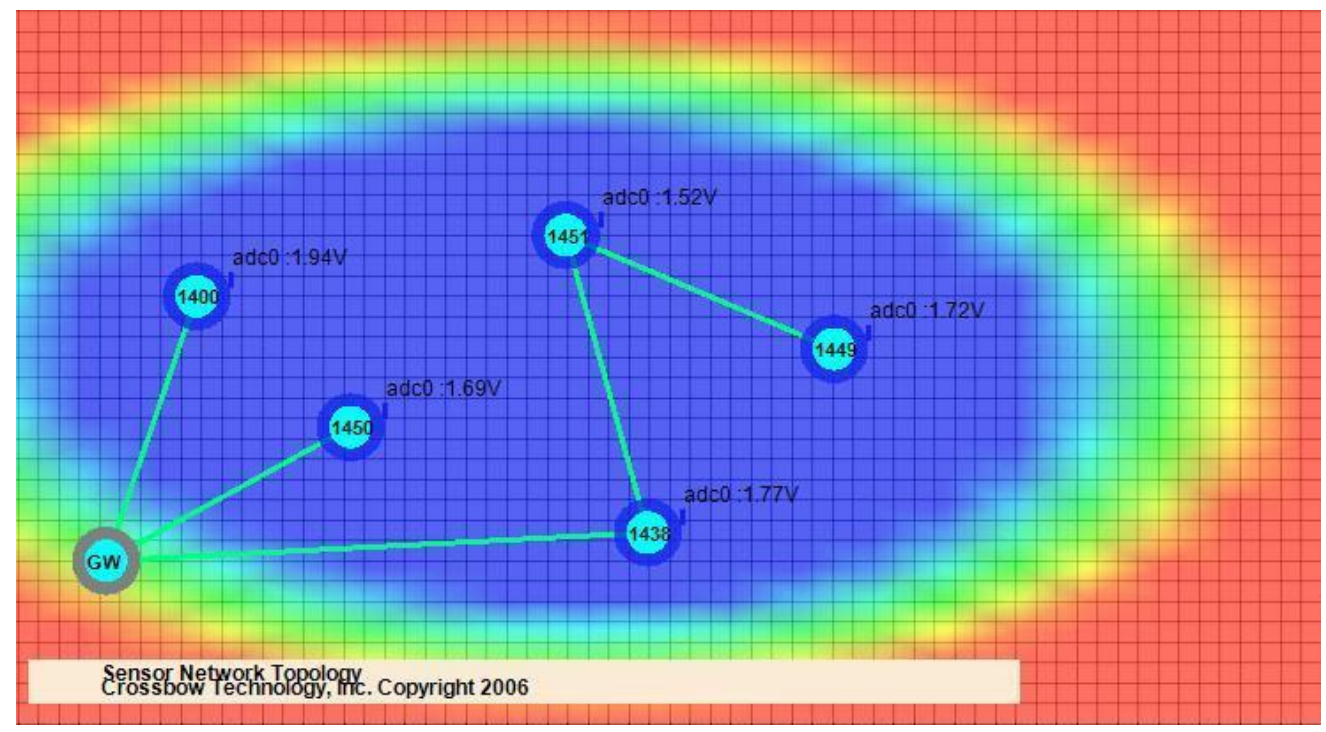

Figure 4.8: Topology 5: Node 1450 Selects Direct Communication with the Base Station as the Best Data Path

Once node 1450 reestablished direct communication with the base station, node 1451 reestablished communication with node 1450 as in the initial setup of the network. Node 1449 continued to transmit through node 1451 . 


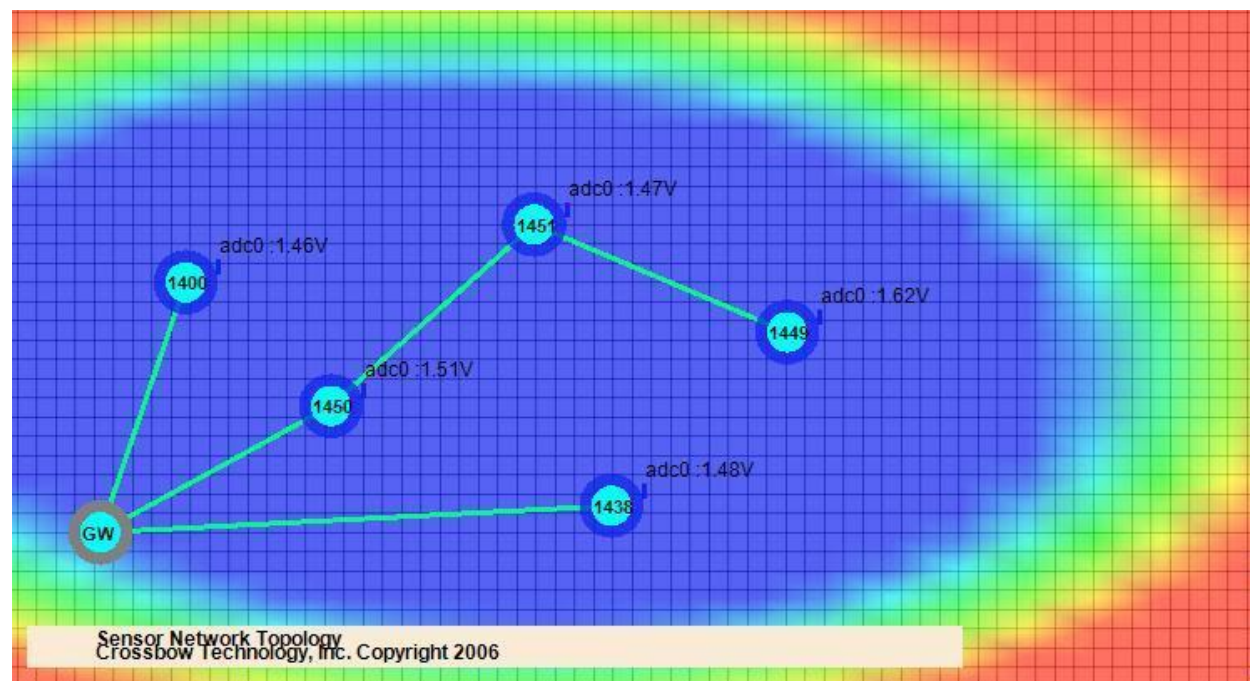

Figure 4.9: Topology 6: Node 1451 Rerouted to Transmit through Node 1450

The final network topology change is shown in Figure 4.10. This topology shows node 1449 selecting another data path and switching to transmit through node 1450.

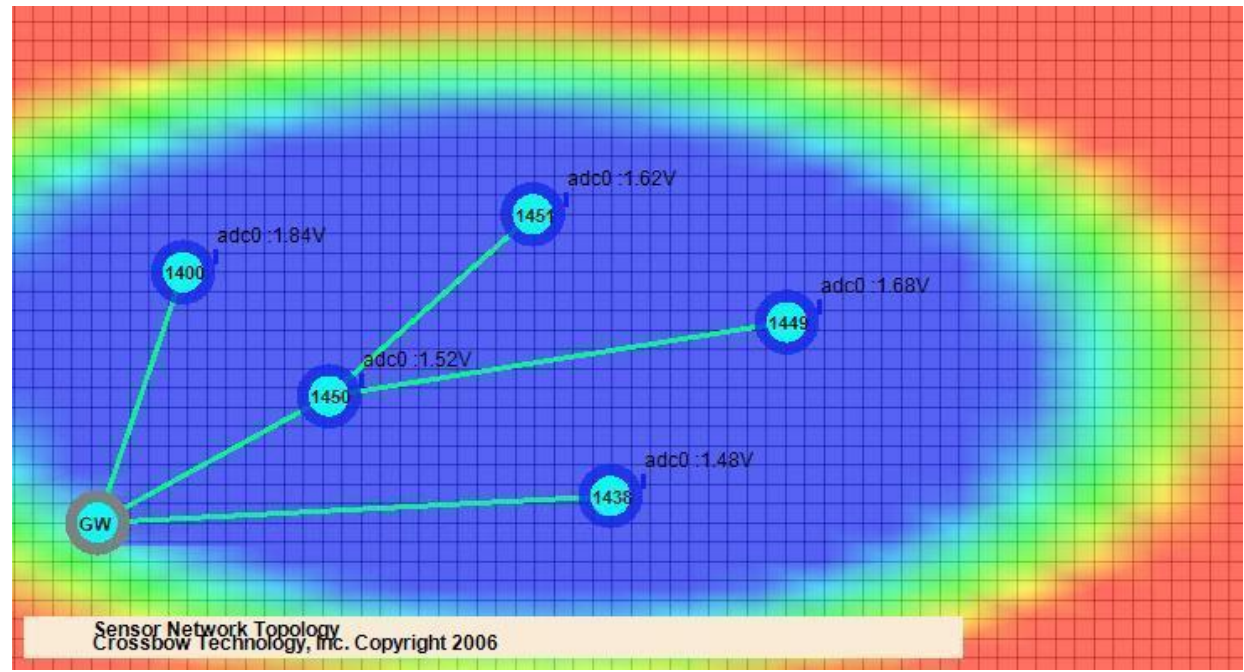

Figure 4.10: Topology 7: Node 1449 Selects Better Data Path and Reroutes through Node 1450 
The various topologies that the network adaptively seeks, while not physically changing the network, is an advantage of the wireless network system as it pertains to network configuration and self-healing abilities.

The intent of the topology test was to test and verify the capabilities of the wireless mesh network. This was done very effectively and as shown through Moteview. The features that were verified were multi-hop, self-configuration, and self-healing. Overall, the topology test was successful in verifying the network features and showing its adaptability and reliability.

\subsubsection{Calibration}

In verifying the capabilities of the network, additional sensors were added along with the power supply boxes to show the versatility of the network. These sensors were calibrated using the various methods described in the Technical Approach. The data recorded and calibration information that was inferred from the data follows in this section.

\subsubsection{Pepperl and Fuchs Ultrasonic Proximity Sensor}

The Pepperl and Fuchs Ultrasonic Proximity Sensor that was used had a sensible range of $0.8 \mathrm{~m}$ to $6 \mathrm{~m}$. This range was converted into feet and a voltage reading was recorded at each foot within the range as shown in Table 4.3. 
Table 4.3: Calibration Data for P\&F Ultrasonic Proximity Sensor

\begin{tabular}{|c|c|}
\hline $\begin{array}{c}\text { Distance } \\
\text { from } \\
\text { Sensor } \\
\text { (in feet) }\end{array}$ & $\begin{array}{c}\text { Voltage Output } \\
\text { to Computer } \\
\text { (in Volts) }\end{array}$ \\
\hline 2.58 & 0.31 \\
\hline 4 & 0.41 \\
\hline 5 & 0.49 \\
\hline 6 & 0.56 \\
\hline 7 & 0.64 \\
\hline 8 & 0.71 \\
\hline 9 & 0.78 \\
\hline 10 & 0.85 \\
\hline 11 & 0.93 \\
\hline 12 & 1 \\
\hline 13 & 1.07 \\
\hline 14 & 1.1409 \\
\hline 15 & 1.2132 \\
\hline 16 & 1.2855 \\
\hline 17 & 1.37 \\
\hline 18 & 1.44 \\
\hline 19 & 1.5 \\
\hline 20 & 1.55 \\
\hline
\end{tabular}

The data was then plotted as shown in Figure 4.19 and a trend line was generated. This trend line was plotted with an equation for the line. The distance component, $\mathrm{x}$, was then solved for and the resulting equation was programmed into Moteview to display a distance in feet rather than a voltage. 


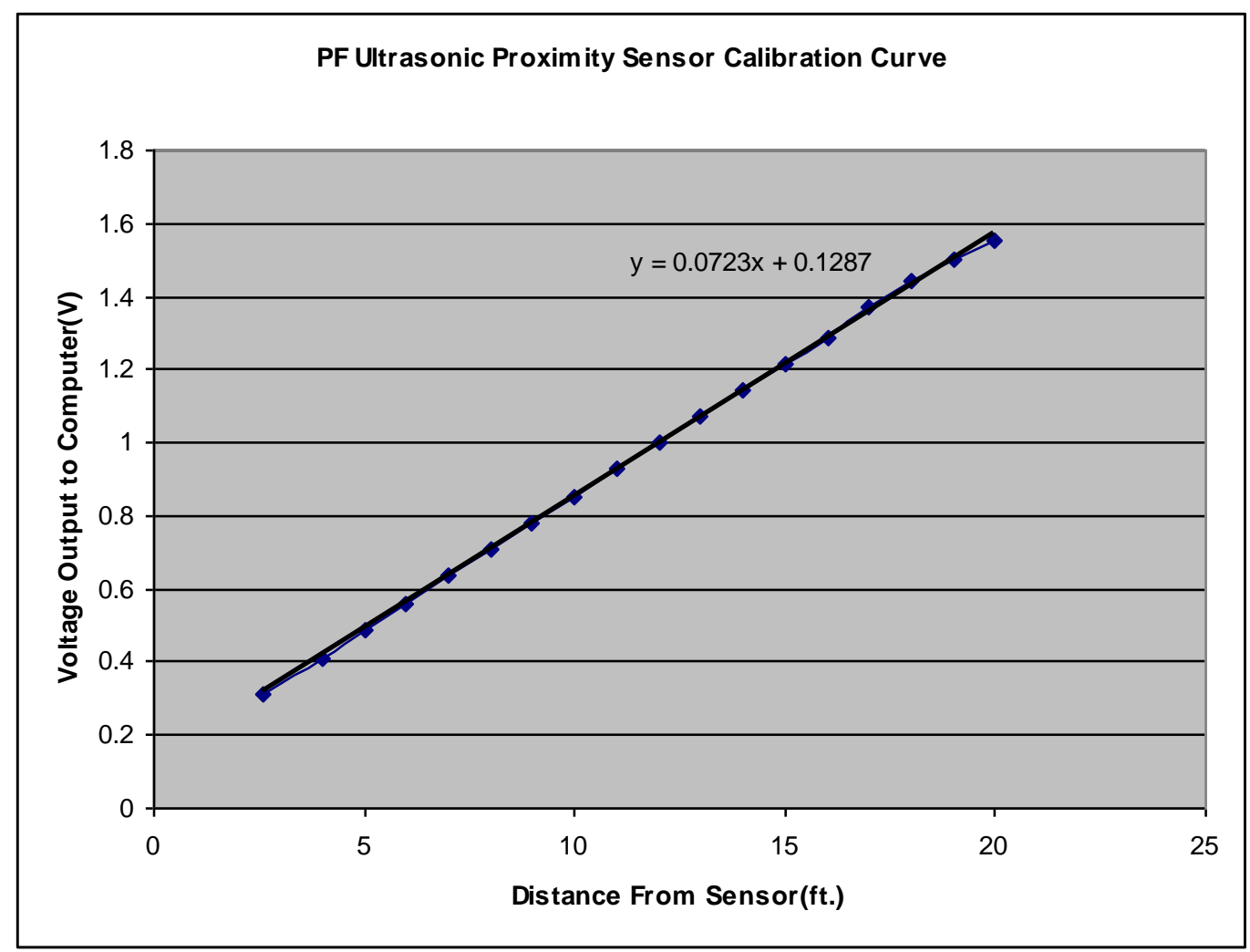

Figure 4.11: P\&F Ultrasonic Proximity Sensor Calibration Curve

\subsubsection{American Sensor Technology Submersible Pressure Transducer / Dwyer Non-Submersible Pressure Transducer / Unimeasure String Potentiometer}

All three of the sensors for this section, the submersible and nonsubmersible pressure transducers, and the string potentiometer were all factory calibrated. The calibration consisted of verifying the data provided by the vendor. After all of the calibration curves were entered into Moteview, all of the sensors all of the output readings were verified. 


\subsubsection{Demonstration Data}

The demonstration staged at the West Virginia University Hangar was intended only as a demonstration of the system functionality. The data obtained and reported in this section is not intended to be interpreted as a real system. Actual implementation would not include the string potentiometer or nonsubmersible pressure transducer. The ultrasonic proximity sensor and submersible pressure transducers could be used an actual system, but again the data reported from these sensors is for demonstration purposes only.

During the small scale demonstration, data was successfully recorded from all four of the sensors that were used. A sample of the raw data can be found in Appendix A. This data was exported into Excel and interpreted to produce Figures 4.12 to 4.16 which represent the data in a more understood manner. Figure 4.12 shows a plot of the total movement or deflection of the inflatable plug in the tunnel as measured by the string potentiometer. 


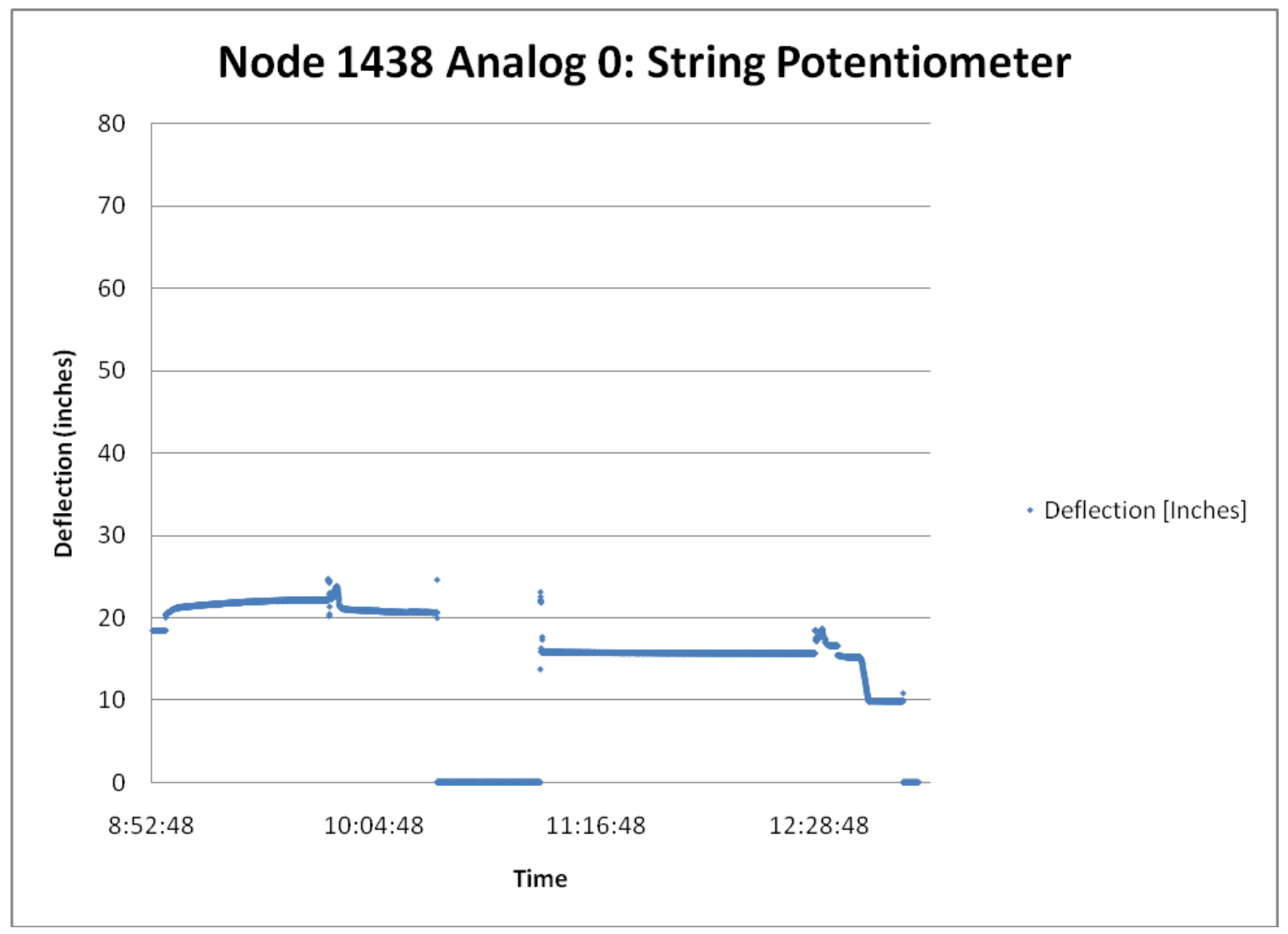

Figure 4.12: Node 1438 Analog 0: String Potentiometer Data

The sections of the plot that remains constant for a long period of time represent the output reading of the string potentiometer prior to the deployment of the plug. When the data begins moving, the plug has been deployed and internal and external pressures are being applied as shown in the following plots. The portion of the plot that goes to zero represents a period of time while the string potentiometer was disconnected to reposition the mechanical plug not a sensor failure.

The non-submersible pressure transducer that was placed on the drainage tube from the inflatable plug shows the internal pressure of the plug as the demonstration was performed. 


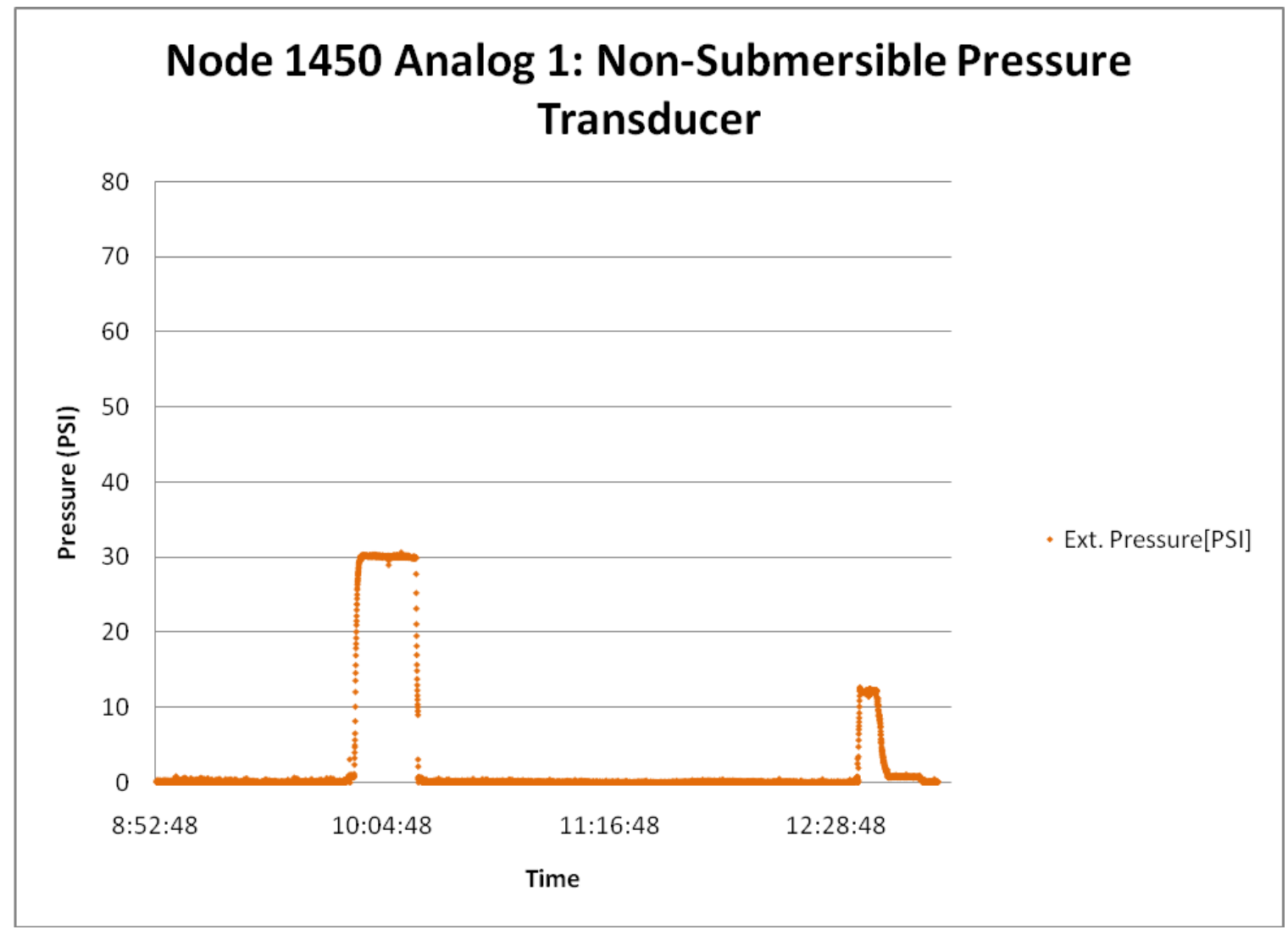

Figure 4.13: Node 1438 Analog 1: Non-Submersible Pressure Transducer Data

The portions of the data at zero represent pauses in the demonstration where an internal pressure was not applied to the mechanical plug. The two peaks of data represent applied pressure to the plug.

Data taken from Node 1450 is shown in Figure 4.14. Node 1450 was used to monitor the external pressure applied to one side of the inflatable plug and the corresponding depth of water. This was done using the non-submersible pressure transducer and the submersible pressure transducer, respectively. 


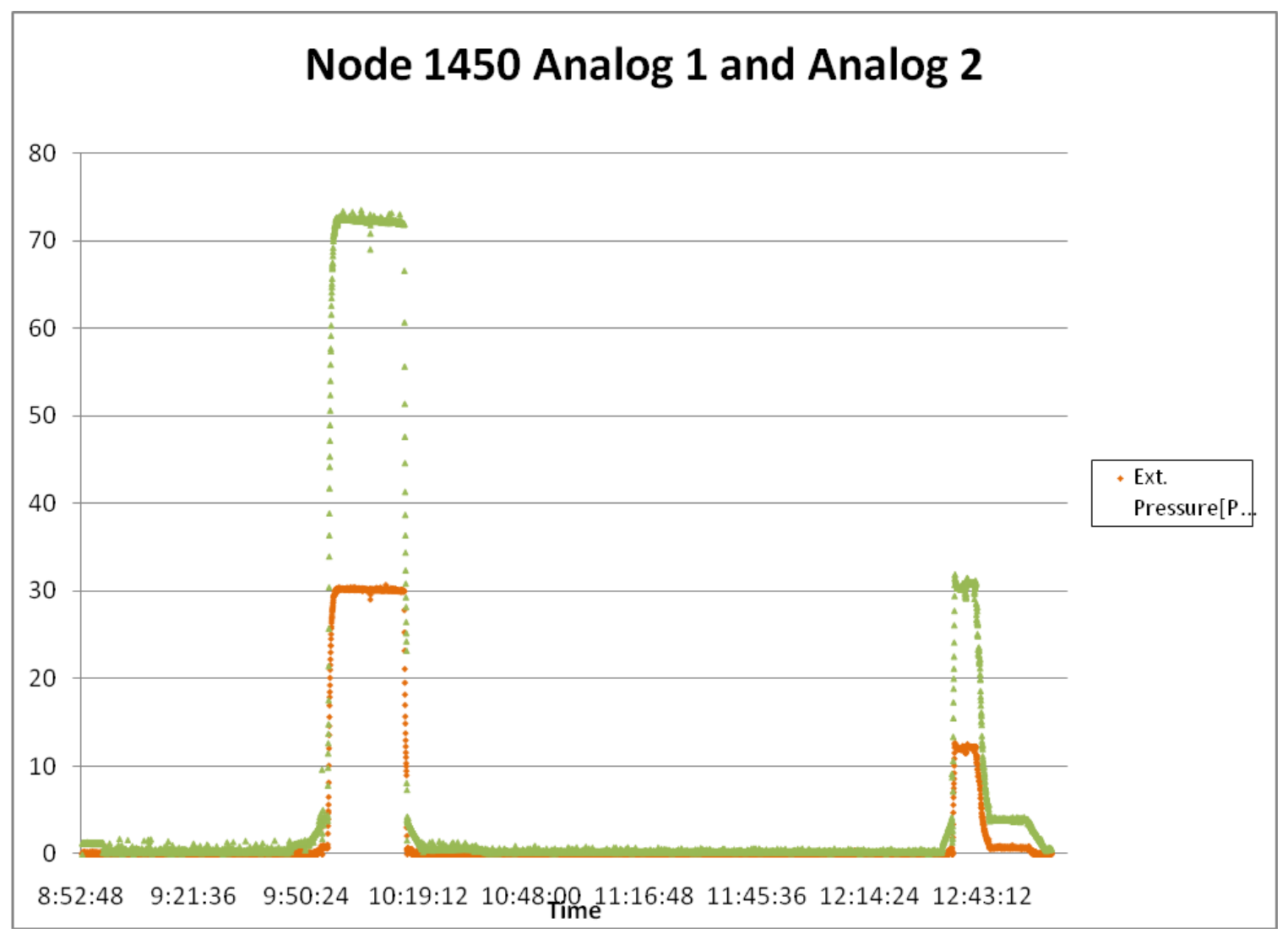

Figure 4.14: Node 1450 Pressure and Water Depth Data

Again, the data that remains at the zero mark in the figure does not represent a sensor failure. The peaks show the measured pressure within the tunnel applied to the outer surface of the plug. The other plot shows the corresponding depth (in feet) for each pressure reading.

Data from node 1438 and node 1450 were then combined to produce Figure 4.15. This figure shows the relationship between the external pressure of the inflatable plug and its movement in the tunnel. This was captured using both nodes and the non-submersible pressure transducer and the string potentiometer. 


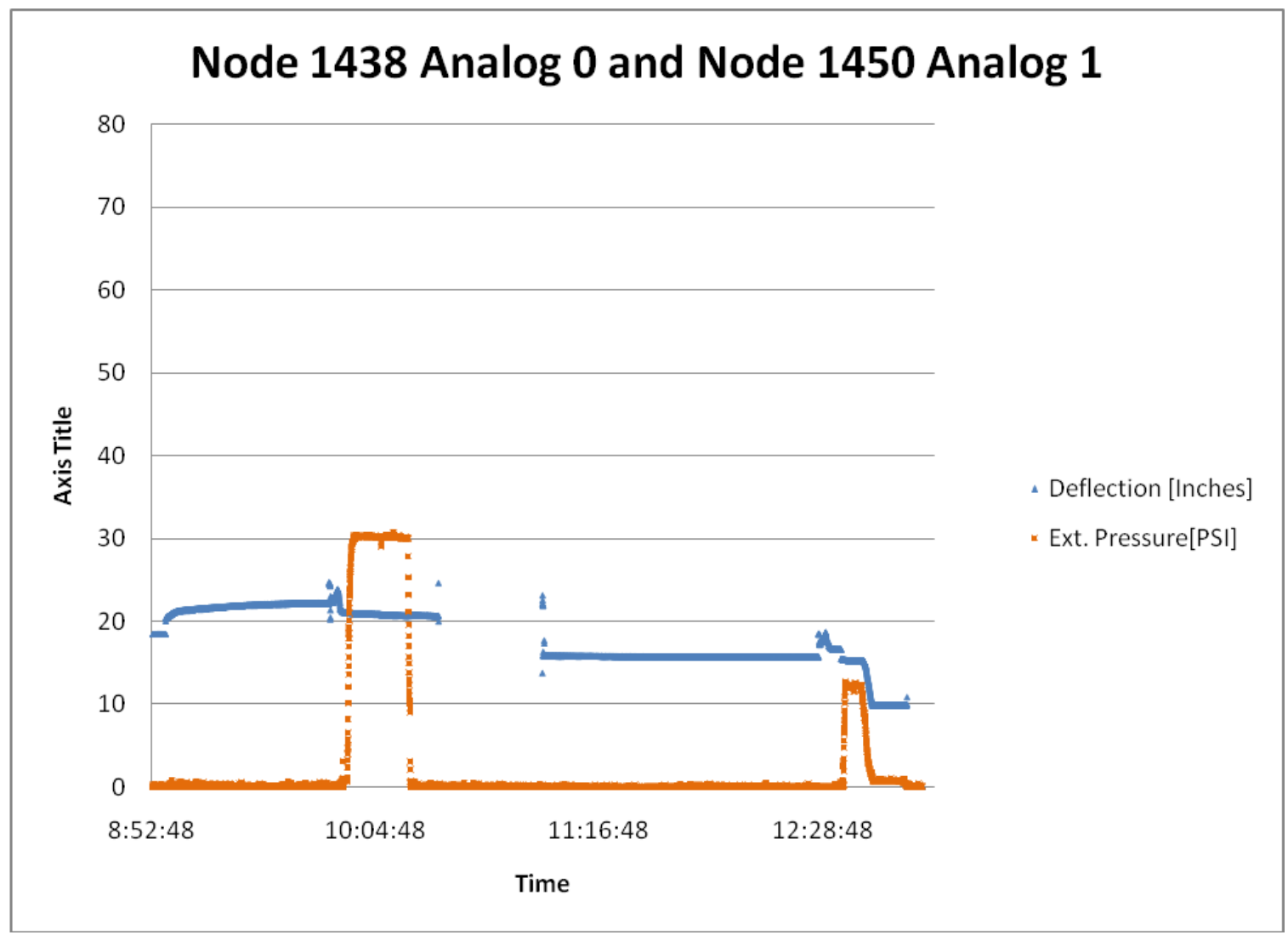

Figure 4.15: Deflection and Pressure Data from Both Nodes

The plot above shows the overlay between the movement/deflection of the plug as it relates to the external pressure applied to the plug. The portions at zero are not failures and are described earlier in the portion of the demonstration data. This shows the plug moves slightly as the external pressure is applied to the plug.

Finally, all node data was combined to observe the entire scheme of the demonstration. Figure 4.16 shows the correspondence between the internal and external pressures of the plug as well as the deflection caused by various depths of water along with other possible combinations of relationships. 


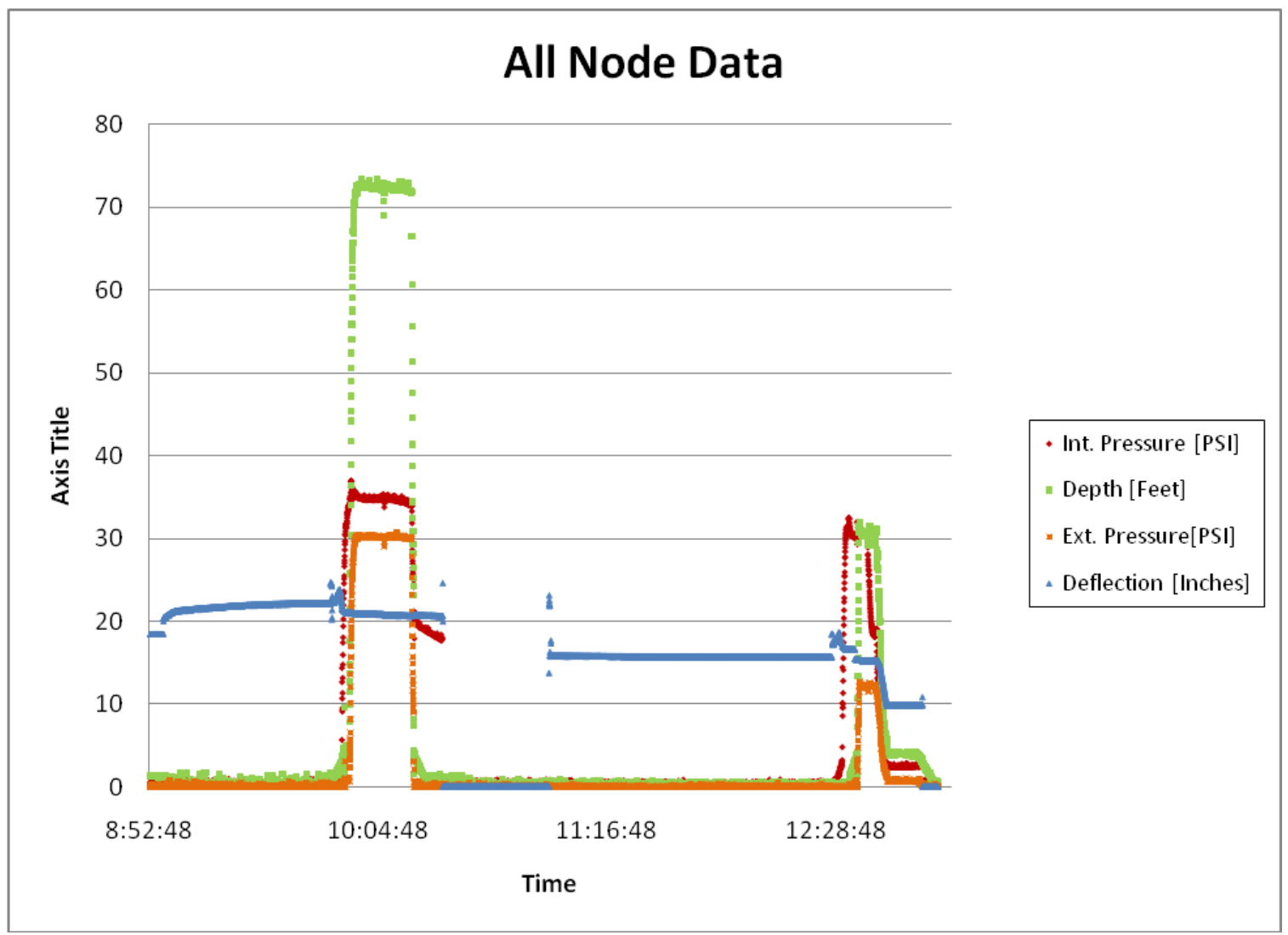

Figure 4.16: All Node Data from Small Scale Demonstration

Descriptions of the data in Figure 4.16 are all contained in the earlier sections of this section and are overlaid with all of the sensor data to show the relationship between all four sensors used.

The demonstration was successful in showing the network's versatility by using external sensors to monitor various desired conditions. The power supplies, external sensors, and wireless nodes all worked properly and data was collected and transmitted to the base station. 


\section{Chapter 5: Conclusion}

With the growing interest in developing a system capable of monitoring the health of an underground structure, the research and design of such a system is the next step in determining the feasibility of such a system. The objective of the research performed was to take that step and develop a system capable of monitoring the health of an underground structure. In order to initiate the process, requirements of the system or network to be developed were set so that the research and design would have some guidance. The research that followed from the requirements led to the selection of a wireless network which utilizes the Zigbee protocol. Various vendors offering hardware which makes use of the selected Zigbee technology were then researched. Crossbow was selected as the most suitable vendor and components for the network were purchased. Following the acquisition of the hardware, a range test, power consumption test, and a data latency test were performed as design tests. An alarm test, topology test, calibration processes, and a small scale demonstration were also performed, but were treated as system verification tests. 


\subsection{Conclusion from Each Test}

\subsubsection{Range}

The range test, which was performed as described earlier, resulted in data that did not display a noticeable difference in transmission reliability for ranges between $300 \mathrm{~m}$ and $500 \mathrm{~m}$. From the data, $500 \mathrm{~m}$ was set as the range at which no redundancy is achieved. In order to achieve single redundancy, it was determined that the nodes would have to be placed $250 \mathrm{~m}$ apart. It is suggested that the nodes be placed between $150 \mathrm{~m}$ and $175 \mathrm{~m}$ apart in order to achieve double redundancy. Spacing the nodes at this interval would allow for more reliable communications both before and after a destructive event as well as a better ability to localize the damage.

\subsubsection{Battery}

After designing and building the power supply boxes, power consumption tests were performed. Low-power mode and high-power mode were both tested for wireless node and it was determined that the wireless node would not be the limiting factor in a scenario where AC power is lost. If the wireless sensor network is set up with five or six sensors at each node, the batteries supplying power to the external sensors would be the limiting factor. In that case, three days to a week would be the expected life of a wireless node due to its external sensor suite. Nearly a week of functionality following the loss of AC power should 
be sufficient time for damage control, emergency response systems, and rescue efforts to have taken place.

\subsubsection{Data Latency}

The results from the data latency test showed reacquisition times of 40 and 60 seconds for the first and second node respectively. This will allow for proper assessment of the situation and a decision to be made regarding actions to be taken. As mentioned, this does not allow for localization of something as fast moving as a blast from an explosion or train pressure wave, but it does allow for localization of slower things such as a fire or flooding which is satisfactory.

\subsubsection{Alarm}

Alarm testing verified the capability of the software to provide notification of a threshold that has been exceeded. An alarm feature could be utilized to alert the appropriate people that a threshold has been broken and allow for prompt action to be taken if necessary. With an alarm being triggered, a more concrete understanding of the situation is presented and less opportunity for interpretation is allowed. 


\subsubsection{Topology}

The intent of the topology test was to test and verify the capabilities of the wireless mesh network. This was done by monitoring the data paths of the network using Moteview. Dynamic routing or frequency hopping were not able to be verified, but there was no noise or interference encountered throughout the testing. Initial setup of the network showed the ability for the network to set itself up by utilizing the self-configuration feature. The self-healing and multi-hop features were verified at various steps throughout the topology test as shown in the Results section. Overall, the topology test was successful in verifying the network features and showing its adaptability and reliability.

\subsection{Calibration}

Though the calibration process was not directly related to the wireless portion of the network, the various external sensors that were connected to the DAQ boards showed the versatility of the sensor portion of the network. Being able to connect a variety of sensors to the wireless network allows it to be applicable to many different structures and scenarios.

\subsection{Demonstration}

The small scale demonstration that was performed was not critical to the design of the network, but it provided the opportunity verify the design and 
demonstrate an example of a possible use. The demonstration utilized the wireless network, the DAQ boards, the external sensors, and the power supply boxes. Verification of the wireless sensor network as a whole was achieved by showing the various sensors that could be used while operating through nodes of the wireless mesh network adhering to the Zigbee protocol. In addition, the power supply boxes allowed for the absence of $A C$ power during the demonstration.

\subsection{Selection of a Sensor for Measuring Water Level}

After the proximity sensor and submersible pressure transducer had been connected to the DAQ boards and tested, the overall feasibility of use and applications of use were analyzed. Since the proximity sensor and water level transducer would essentially be monitoring the same thing, water depth, a suggested selection was determined. The submersible pressure transducer is the suggested sensor based on the facts that it is more robust, and overall more suited to the application of monitoring water levels. However, certain applications could potentially not allow for the pressure transducer to be placed at the bottom of the structure where it needs to be. Any application with this scenario would be more suited to use the proximity sensor and mount it on the ceiling or roof of the structure. While the submersible pressure transducer is the suggested sensor to be used, this does not mean the proximity sensor is rejected or shouldn't be used for the exact same application. 


\subsection{Feasibility for Application}

Following all of the testing, the feasibility of the overall design of the network was considered and all of the necessary requirements for this network to be implemented were met meaning the network provides complete, redundant, reliable communication throughout a structure, successfully monitors desired conditions, and can be easily retrofitted or customized. Overall, the wireless sensor network that was designed is a feasible approach to satisfying the need for a system to monitor the health of underground structures with the additional appeal of being able to retrofit or customize the network.

\subsection{Further Studies}

Approaching the design from the mechanical and physical point of view allows for future work in honing the existing network design by examining some of the smaller components on the computer and electrical level. In order to fully understand the underlying technology and smaller components it is suggested that the network be looked at from the computer/electrical perspective.

Additional work to be completed to better the design of the network include work with the digital outputs of the DAQ boards, interfacing with emergency systems, and a more applicable user interface. The digital outputs can be used for a number of additional features for the network. They could be used to actuate a mechanical component of the system, such as the inflatable 
plug in the demonstration. Another possible use would be to control relays to turn external sensors on and off to save power. A third possible suggestion would be to use the digital outputs to actuate an emergency system such as lighting, guide beacons, or flood/fire suppression.

\subsubsection{User Interface}

The final design portion of this project, the operator interface, will require future work from an individual skilled in graphical user interface programming. The operator interface will provide a simple interpretation of what is happening at each node throughout the tunnel and will be programmed with thresholds and alarms to make user decision making more concise.

The desired operator interface is discussed below with illustrations to more clearly show the design. The operator interface is discussed for a tunnel, but could be easily interpreted to be applied to a mine or other structure.

The front page or standard display for the operator interface will consist of a side-view of the tunnel with nodes shown throughout the structure. The nodes will be shown in green if they are "healthy" and no thresholds have been exceeded. If the cursor is moved over one of the nodes, a small bubble pops up with the most recent sensor outputs. This is depicted in Figure 5.1. 


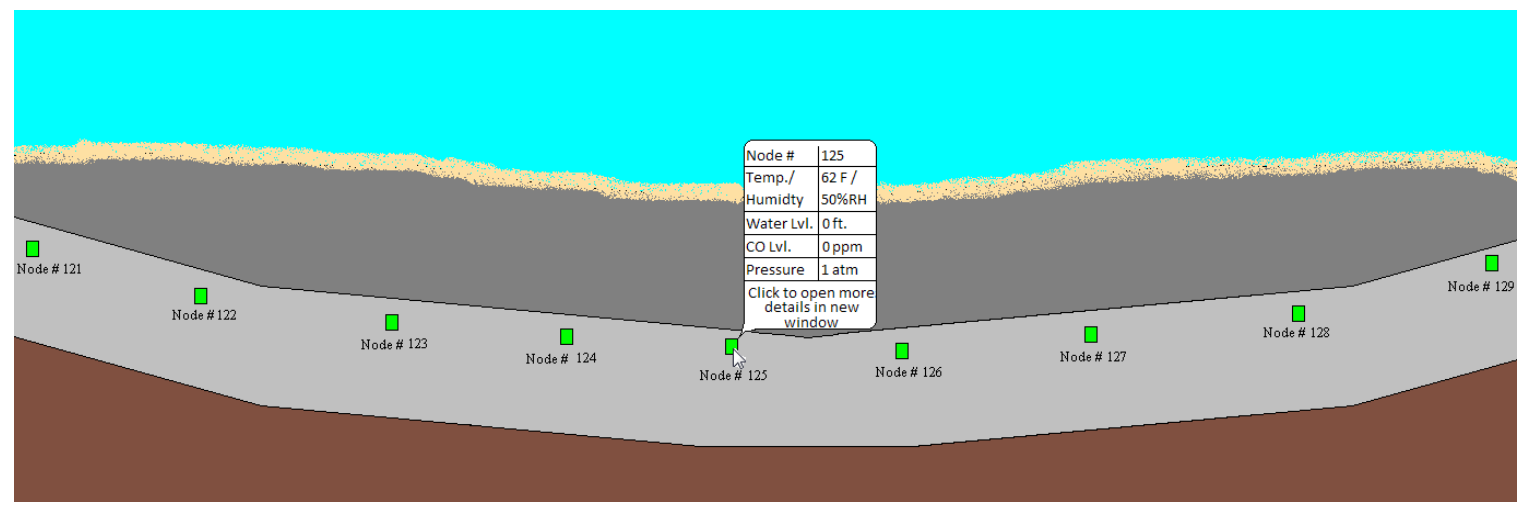

Figure 5.1: Example of Operator Interface Under Normal Conditions

The figure above shows the tunnel section under normal conditions with no thresholds being exceeded. The figure below shows a flooding scenario where the lowest portion of the tunnel is flooding.

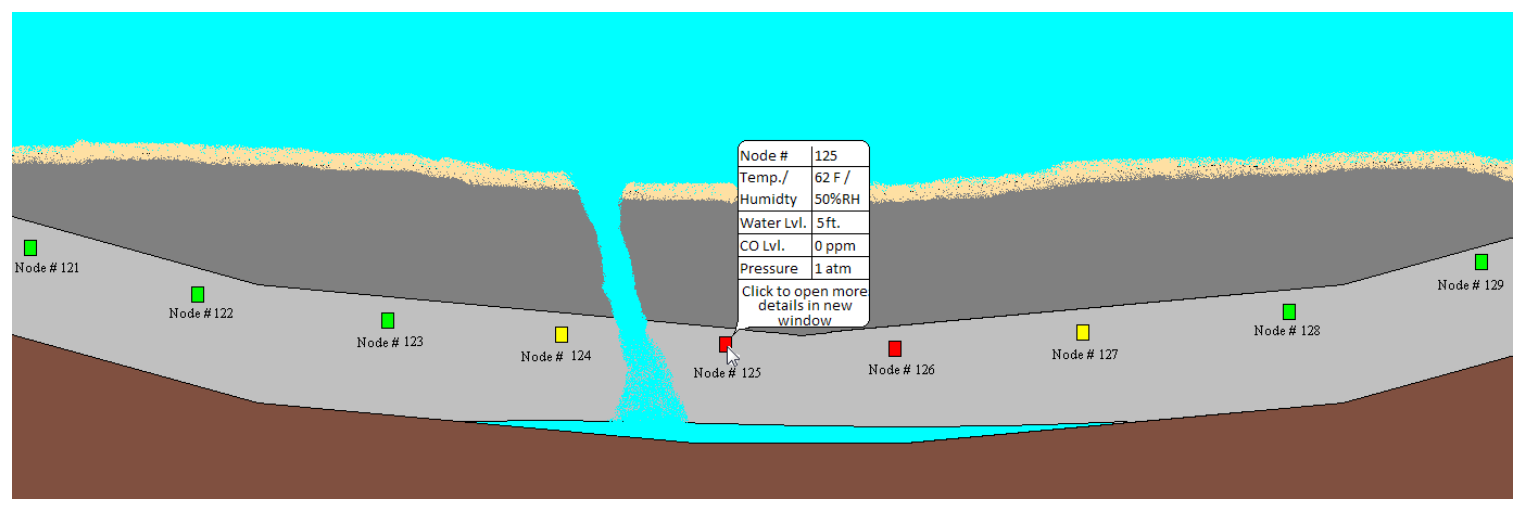

Figure 5.2: Example of Operator Interface Under Flooding Conditions

The nodes in the affected area have turned red, indicating a threshold has been exceeded and actions need to be taken. The nodes to the outer reaches of the flood water have turned yellow indicating a change in water level that has not 
yet reached a critical limit. If the node is clicked on, a separate window will pop up with more detailed sensor outputs over a longer period of time as the following figure illustrates.

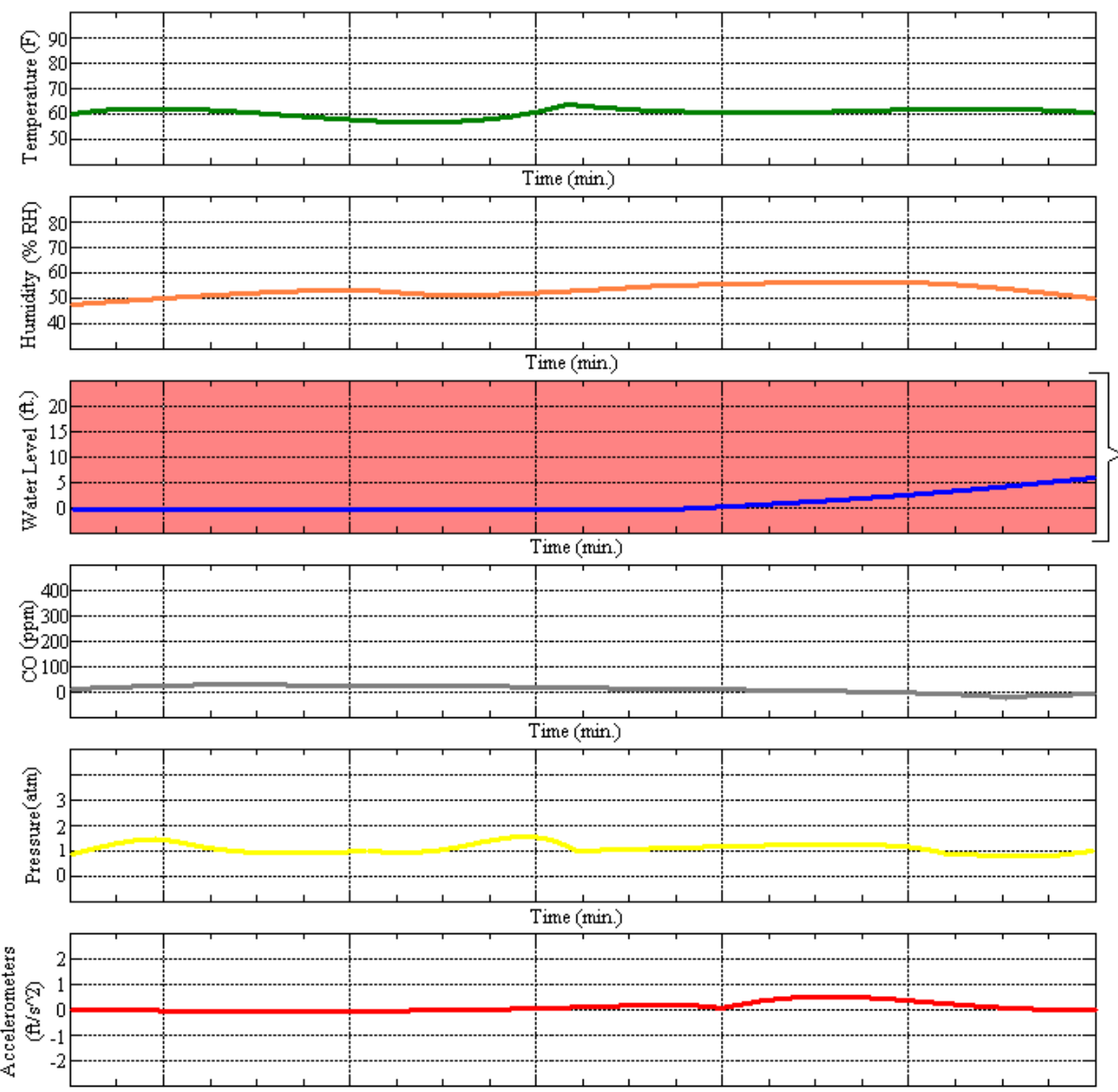

Threshold has been exceeded. Click below for a list of actions to be taken.

Actions

Click and drag to zoom on any section of data.

Figure 5.3: Detailed Data Display from Single Node with Alarm

The user will have the ability to click and drag a box around any area on the data shown to enlarge it and have a more detailed view of the data. In the simulated data above, the water level has exceeded a critical limit and requires 
actions to be taken. This is shown by a red tint to the plot as well as the pop up bubble stating "Threshold has been exceeded. Click for a list of actions to be taken." The operator should then click the "Actions" button for a list of possible actions to be performed. The figure below shows the designed window that will appear if the operator clicks on the "Actions" button.

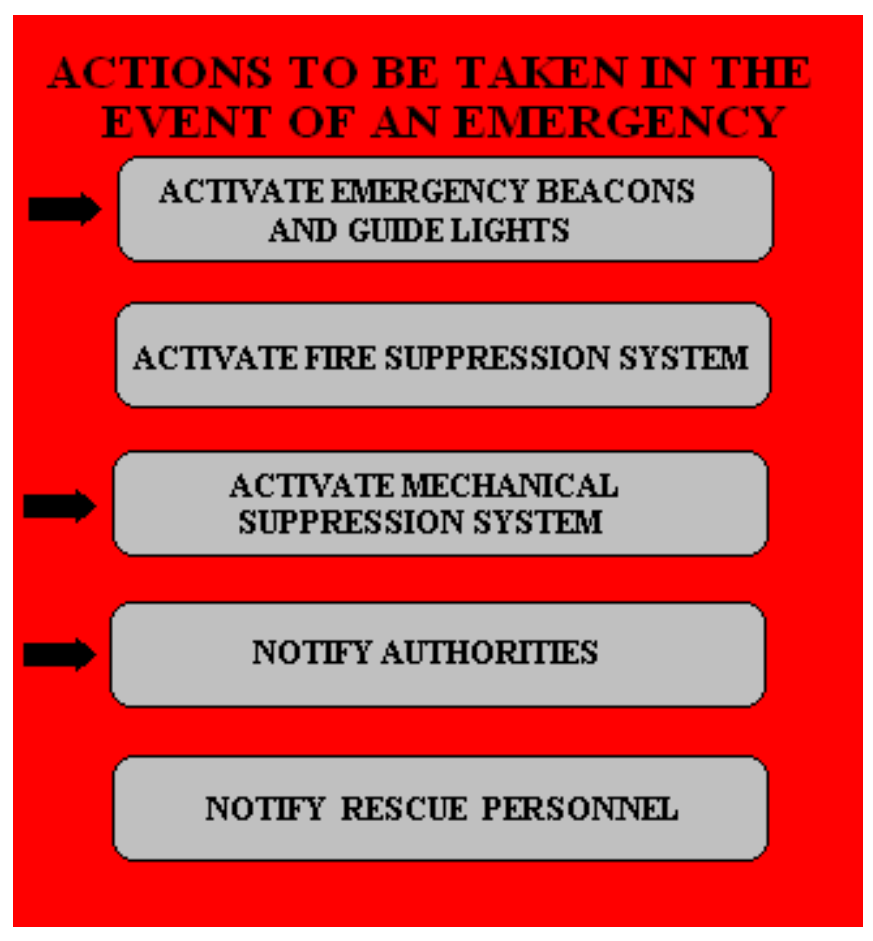

Figure 5.4: Possible Actions to be Taken in the Event of an Emergency

The buttons in the above figure give examples of potential actions to be taken if a scenario calls for it. Depending on the threshold that has been broken, a black arrow will appear next to the suggested actions. In the scenario of flooding, "Activate emergency beacons and guide lights," "Activate mechanical suppression system," and "Notify authorities" are suggested actions. In the event 
of a fire, "Activate fire suppression system" would be the suggested action. If hazardous conditions become present in the tunnel, "Notify rescue personnel" would become a suggested action. These buttons can be modified or added to depending on the functionality of the sensor network.

This operator interface supplies simple graphical representations of the conditions throughout the tunnel and walks the operator through each step depending on the severity of the situation. This will allow for prompt decisions to be made and actions to be taken when necessary. 


\section{Bibliography}

[1] Brambley, Michael. "Wireless Sensors and Controls for Buildings." Pacific Northwest National Laboratory. 16 January 2003. 26 January 2009 <http://www.pnl.gov/wireless-buildings/cost.htm\#ln\%20Building>

[2] "ETRX2 ZigBee Module". Telegesis.

09/24/09<http://www.telegesis.com/products/test_page_1.htm>.

[3] "Fatal Underground Coal Mine Explosion ". Mine Safety and Health Administration(MSHA). 07/08/09 <http://www.msha.gov/Fatals/2006/Sago/sagoreport.asp>.

[4] Findlater, L.. "Quecreek Mine Flooding Disaster". Mine Safety and Health Administration(MSHA). 07/08/09 $<$ http://technology.infomine.com/articles/1/632/mine.flood.quecreek/ quecreek.mine.flooding.aspx>.

[5] Fleck, Fiona. "80 Still Missing in Swiss Tunnel Crash". Daily mail. 07/08/09 $<$ http://www.dailymail.co.uk/news/article-80452/80-missing-Swisstunnel-crash.html>.

[6] Hall, Joseph. "Ten Years After". Toronto Transit Commission. 07/12/09 <http://transit.toronto.on.ca/archives/data/200508061136.shtml>.

[7] Hanlin, Jack and Derek Walker. "Wi-Fi." 10 July 2009. 26 January 2009 <http://searchmobilecomputing.techtarget.com/sDefinition/0,,sid 40_gci838865,00.html>

[8] Helicomm. 09/24/09 <http://www.helicomm.com/products.asp?BigClass $=$ Embedded + Module $\&$ SmallClass $=2 \% 2 E 4 G H z+Z i g B e e+$ Module $>$.

[9] "How Bluetooth Technology Works." Bluetooth. 26 January 2009 <http://www.bluetooth.com/Bluetooth/Technology/Works/ > . Multiple Subsections.

[10] "How Bluetooth Works." GSMFavorites.com. 26 January 2009 <http://www.gsmfavorites.com/documents/bluetooth/works/ >

[11] "IEEE Standard Extends Wireless Communications to Low Power, LowSpeed, Low-Cost Devices: IEEE 802.15.4 Expected to Foster Wireless Use of Sensor and Actuator Network." 2 September 2003. 2 February 2009 <http://standards.ieee.org/announcements/pr_802154.html> 
[12] IEEE Std. 802.15.4-2006, IEEE Standard for Information Technology Telecommunications and Information Exchange Between Systems - Local and Metropolitan Area Networks - Specific Requirements Part 15.4: Wireless Medium Access Control(MAC) and Physical Layer(PHY) Specifications for Low-Rate Wireless Personal Area Networks(WPANS)

[13] "Industrial Sensing the Wireless Way." Machine Design.com: By Engineers for Engineers. 6 January 2005. 26 January 2009 $<$ http://machinedesign.com/article/industrial-sensing-the-wirelessway-0106>

[14] "IRIS Wireless Measurement System". Crossbow. 09/24/09 $<$ http://www.xbow.com/Products/Product_pdf_files/ Wireless_pdf/IRIS_Datasheet.pdf>.

[15] Kim, Susan. "Explosions Stun London". Disaster News Network. 7/16/09 <http://www.disasternews.net/news/article.php?articleid=3027>.

[16] Martin, Christopher. "Six Coal Miners Trapped in Utah". United States Mine Rescue Association. 07/12/09 <http://www.usmra.com/saxsewell/crandallcanyon.htm>.

[17] Merrit, Rich. "Mesh Networks on the Factory Floor." Control Global. 26 January 2005. 2 February 2009 <http://www.controlglobal.com/articles/2005/313.html >

[18] Riding, Alan. "Toll Rises to 35 in Mont Blanc Tunnel Fire". New York Times. 07/08/09 <http://www.nytimes.com/1999/03/27/world/ toll-rises-to-35-in-mont-blanc-tunnel-fire.html>.

[19] "TinyOne Zigbee 2.4 Ghz OEM RF". One RF. 09/24/09 $<$ http://pdf.directindustry.com/pdf/telit/tinyone-zigbee-24-ghz-oemrf-module/35043-9997-_2.html>.

[20] United States. Center for Disease Control Office of Mine Safety and Health. Communications and Tracking Research. 3 May 2007. 3 February 2009 $<$ http://www.cdc.gov/niosh/mining/commtrack/pdfs/mshrac050307.p df $>$

[21] United States. Mine Safety and Health Administration. Mine Improvement and New Emergency Response Act of 2006. 15 June 2006. 4 February 2009 <http://www.msha.gov/MinerAct/2006mineract.pdf> 
[22] United States. Mine Safety and Health Administration. Underground Mine Rescue Equipment and Technology; Proposed Rule. 7 December 2007. 6 July 2009 <http://www.msha.gov/sagomine/sagomine.asp $>$

[23] "What is Ethernet?". University of Indiana Information Technology Services Knowledge Base. 13 May 2009. 24 January 2009 $<$ http://kb.iu.edu/data/aesi.html>

[24] "XBee/XBee-Pro OEM RF Modules". XBee. 09/24/09 $<$ http://www.sparkfun.com/datasheets/Wireless/Zigbee/XBeeManual.pdf>.

[25] "ZigBee Products". RFM. 09/24/09 $<$ http://www.rfm.com/products/zigbee.php>. 
Appendix A (Raw Data) 


\section{Samples of Data from Demonstration}

\begin{tabular}{|l|l|r|r|r|r|r|r|r|r|r|r|}
\hline Id & Time & parent & voltage [V] & adc0 [Inches] & adc1 [PSI] & adc2 [Feet] & adc3 [V] & digi0 & digi1 & digi2 & digi3 \\
\hline 1438 & $6 / 9 / 20098: 53$ & 0 & 2.53 & 18.446 & 0.56763 & 0.46752 & 0 & 1 & 1 & 1 & 1 \\
\hline 1438 & $6 / 9 / 20098: 53$ & 0 & 2.53 & 18.445 & 0.67673 & 0.47861 & 0 & 1 & 1 & 1 & 1 \\
\hline 1438 & $6 / 9 / 20098: 53$ & 0 & 2.53 & 18.453 & 0.59738 & 0.45643 & 0 & 1 & 1 & 1 & 1 \\
\hline 1438 & $6 / 9 / 20098: 53$ & 0 & 2.53 & 18.446 & 0.59509 & 0.46309 & 0 & 1 & 1 & 1 & 1 \\
\hline 1438 & $6 / 9 / 20098: 53$ & 0 & 2.53 & 18.445 & 0.59433 & 0.47861 & 0 & 1 & 1 & 1 & 1 \\
\hline 1438 & $6 / 9 / 20098: 53$ & 0 & 2.53 & 18.446 & 0.59357 & 0.47861 & 0 & 1 & 1 & 1 & 1 \\
\hline 1438 & $6 / 9 / 20098: 53$ & 0 & 2.53 & 18.448 & 0.61722 & 0.49192 & 0 & 1 & 1 & 1 & 1 \\
\hline 1438 & $6 / 9 / 20098: 53$ & 0 & 2.53 & 18.448 & 0.60806 & 0.47196 & 0 & 1 & 1 & 1 & 1 \\
\hline 1438 & $6 / 9 / 20098: 53$ & 0 & 2.53 & 18.447 & 0.56534 & 0.45422 & 0 & 1 & 1 & 1 & 1 \\
\hline 1438 & $6 / 9 / 20098: 53$ & 0 & 2.53 & 18.446 & 0.64774 & 0.45643 & 0 & 1 & 1 & 1 & 1 \\
\hline 1438 & $6 / 9 / 20098: 53$ & 0 & 2.53 & 18.446 & 0.53864 & 0.48305 & 0 & 1 & 1 & 1 & 1 \\
\hline 1438 & $6 / 9 / 20098: 53$ & 0 & 2.53 & 18.446 & 0.66757 & 0.47418 & 0 & 1 & 1 & 1 & 1 \\
\hline 1438 & $6 / 9 / 20098: 53$ & 0 & 2.53 & 18.448 & 0.55618 & 0.46087 & 0 & 1 & 1 & 1 & 1 \\
\hline 1438 & $6 / 9 / 20098: 53$ & 0 & 2.53 & 18.447 & 0.5394 & 0.49635 & 0 & 1 & 1 & 1 & 1 \\
\hline 1438 & $6 / 9 / 20098: 53$ & 0 & 2.53 & 18.445 & 0.56534 & 0.48748 & 0 & 1 & 1 & 1 & 1 \\
\hline 1438 & $6 / 9 / 20098: 53$ & 0 & 2.53 & 18.447 & 0.58517 & 0.49635 & 0 & 1 & 1 & 1 & 1 \\
\hline 1438 & $6 / 9 / 20098: 53$ & 0 & 2.53 & 18.448 & 0.59586 & 0.48526 & 0 & 1 & 1 & 1 & 1 \\
\hline
\end{tabular}

\begin{tabular}{|c|c|c|c|c|c|c|c|c|c|c|c|}
\hline 1438 & 6/9/2009 12:03 & 0 & 2.9398 & 15.68 & 0.35858 & -0.18674 & 0 & 1 & 1 & 1 & 1 \\
\hline 1438 & 6/9/2009 12:03 & 0 & 2.9398 & 15.71 & 0.39215 & 0.03726 & 0 & 1 & 1 & 1 & 1 \\
\hline 1438 & 6/9/2009 12:03 & 0 & 2.9398 & 15.704 & 0.40054 & 0.035042 & 0 & 1 & 1 & 1 & 1 \\
\hline 1438 & 6/9/2009 12:03 & 0 & 2.9398 & 15.678 & 0.49057 & -0.011533 & 0 & 1 & 1 & 1 & 1 \\
\hline 1438 & 6/9/2009 12:03 & 0 & 2.9398 & 15.708 & 0.42953 & -0.11134 & 0 & 1 & 1 & 1 & 1 \\
\hline 1438 & 6/9/2009 12:03 & 0 & 2.9398 & 15.692 & 0.54321 & 0.023953 & 0 & 1 & 1 & 1 & 1 \\
\hline 1438 & 6/9/2009 12:03 & 0 & 2.9398 & 15.684 & 0.33112 & 0.2036 & 0 & 1 & 1 & 1 & 1 \\
\hline 1438 & 6/9/2009 12:04 & 0 & 2.9398 & 15.691 & 0.42648 & -0.0048793 & 0 & 1 & 1 & 1 & 1 \\
\hline 1438 & 6/9/2009 12:04 & 0 & 2.9398 & 15.697 & 0.39597 & -0.035929 & 0 & 1 & 1 & 1 & 1 \\
\hline 1438 & 6/9/2009 12:04 & 0 & 2.9398 & 15.687 & 0.43945 & -0.14682 & 0 & 1 & 1 & 1 & 1 \\
\hline 1438 & 6/9/2009 12:04 & 0 & 2.9398 & 15.657 & 0.36087 & -0.018186 & 0 & 1 & 1 & 1 & 1 \\
\hline 1438 & 6/9/2009 12:04 & 0 & 2.9398 & 15.692 & 0.41885 & 0.079399 & 0 & 1 & 1 & 1 & 1 \\
\hline 1438 & 6/9/2009 12:04 & 0 & 2.9398 & 15.704 & 0.50888 & -0.047018 & 0 & 1 & 1 & 1 & 1 \\
\hline 1438 & 6/9/2009 12:04 & 0 & 2.9398 & 15.687 & 0.37689 & -0.015968 & 0 & 1 & 1 & 1 & 1 \\
\hline 1438 & 6/9/2009 12:04 & 0 & 2.9398 & 15.699 & 0.40054 & -0.011533 & 0 & 1 & 1 & 1 & 1 \\
\hline 1438 & 6/9/2009 12:04 & 0 & 2.9398 & 15.688 & 0.61417 & 0.066092 & 0 & 1 & 1 & 1 & 1 \\
\hline 1438 & 6/9/2009 12:04 & 0 & 2.9398 & 15.701 & 0.50354 & -0.089157 & 0 & 1 & 1 & 1 & 1 \\
\hline 1438 & 6/9/2009 12:04 & 0 & 2.9398 & 15.7 & 0.39597 & -0.044801 & 0 & 1 & 1 & 1 & 1 \\
\hline 1438 & 6/9/2009 12:04 & 0 & 2.9398 & 15.694 & 0.42496 & -0.0048793 & 0 & 1 & 1 & 1 & 1 \\
\hline 1438 & 6/9/2009 12:04 & 0 & 2.9398 & 15.675 & 0.55466 & -0.015968 & 0 & 1 & 1 & 1 & 1 \\
\hline 1438 & 6/9/2009 12:04 & 0 & 2.9398 & 15.663 & 0.39215 & 0.041696 & 0 & 1 & 1 & 1 & 1 \\
\hline 1438 & 6/9/2009 12:04 & 0 & 2.9398 & 15.699 & 0.39215 & -0.0070971 & 0 & 1 & 1 & 1 & 1 \\
\hline 1438 & 6/9/2009 12:04 & 0 & 2.9398 & 15.681 & 0.54703 & 0.063874 & 0 & 1 & 1 & 1 & 1 \\
\hline
\end{tabular}




\begin{tabular}{|r|r|r|r|r|r|r|r|r|r|r|r|}
\hline 1450 & $6 / 9 / 20098: 53$ & 0 & 2.4848 & 3.7605 & 0.13199 & 1.2948 & 0 & 1 & 1 & 1 & 1 \\
\hline 1450 & $6 / 9 / 20098: 53$ & 0 & 2.4848 & 3.756 & 0 & 1.3037 & 0 & 1 & 1 & 1 & 1 \\
\hline 1450 & $6 / 9 / 20098: 53$ & 0 & 2.4799 & 3.7569 & 0 & 1.2815 & 0 & 1 & 1 & 1 & 1 \\
\hline 1450 & $6 / 9 / 20098: 53$ & 0 & 2.4799 & 3.7569 & 0.29068 & 1.297 & 0 & 1 & 1 & 1 & 1 \\
\hline 1450 & $6 / 9 / 20098: 53$ & 0 & 2.4848 & 3.7642 & 0.026703 & 1.2859 & 0 & 1 & 1 & 1 & 1 \\
\hline 1450 & $6 / 9 / 20098: 53$ & 0 & 2.4799 & 3.7527 & 0 & 1.3125 & 0 & 1 & 1 & 1 & 1 \\
\hline 1450 & $6 / 9 / 20098: 53$ & 0 & 2.4848 & 3.756 & 0.15793 & 1.2504 & 0 & 1 & 1 & 1 & 1 \\
\hline 1450 & $6 / 9 / 20098: 53$ & 0 & 2.4799 & 3.7582 & 0 & 1.317 & 0 & 1 & 1 & 1 & 1 \\
\hline 1450 & $6 / 9 / 20098: 53$ & 0 & 2.4799 & 3.756 & 0.028992 & 1.3214 & 0 & 1 & 1 & 1 & 1 \\
\hline 1450 & $6 / 9 / 20098: 53$ & 0 & 2.4799 & 3.7532 & 0.17776 & 1.2992 & 0 & 1 & 1 & 1 & 1 \\
\hline 1450 & $6 / 9 / 20098: 53$ & 0 & 2.4799 & 3.7537 & 0.15259 & 1.3037 & 0 & 1 & 1 & 1 & 1 \\
\hline 1450 & $6 / 9 / 20098: 53$ & 0 & 2.4848 & 3.755 & 0.17853 & 1.2859 & 0 & 1 & 1 & 1 & 1 \\
\hline 1450 & $6 / 9 / 20098: 53$ & 0 & 2.4799 & 3.756 & 0 & 1.3037 & 0 & 1 & 1 & 1 & 1 \\
\hline 1450 & $6 / 9 / 20098: 53$ & 0 & 2.4848 & 3.7679 & 0.18387 & 1.3125 & 0 & 1 & 1 & 1 & 1 \\
\hline 1450 & $6 / 9 / 20098: 53$ & 0 & 2.4848 & 3.755 & 0.103 & 1.2682 & 0 & 1 & 1 & 1 & 1 \\
\hline 1450 & $6 / 9 / 20098: 53$ & 0 & 2.4848 & 3.7482 & 0.011444 & 1.3037 & 0 & 1 & 1 & 1 & 1 \\
\hline 1450 & $6 / 9 / 20098: 53$ & 0 & 2.4799 & 3.7468 & 0.31967 & 1.2859 & 0 & 1 & 1 & 1 & 1 \\
\hline 1450 & $6 / 9 / 20098: 53$ & 0 & 2.4848 & 3.7532 & 0 & 1.3037 & 0 & 1 & 1 & 1 & 1 \\
\hline 1450 & $6 / 9 / 20098: 53$ & 0 & 2.4799 & 3.7587 & 0 & 1.3037 & 0 & 1 & 1 & 1 & 1 \\
\hline 1450 & $6 / 9 / 20098: 53$ & 0 & 2.4848 & 3.7463 & 0.06485 & 1.2815 & 0 & 1 & 1 & 1 & 1 \\
\hline 1450 & $6 / 9 / 20098: 53$ & 0 & 2.4848 & 3.7569 & 0 & 1.2948 & 0 & 1 & 1 & 1 & 1 \\
\hline 1450 & $6 / 9 / 20098: 53$ & 0 & 2.4848 & 3.7523 & 0 & 1.2992 & 0 & 1 & 1 & 1 & 1 \\
\hline
\end{tabular}

\begin{tabular}{|r|r|r|r|r|r|r|r|r|r|r|r|}
\hline 1450 & $6 / 9 / 200911: 23$ & 0 & 3.1948 & 3.8136 & 0 & 0.28122 & 0 & 1 & 1 & 1 & 1 \\
\hline 1450 & $6 / 9 / 200911: 23$ & 0 & 3.1948 & 4.1771 & 0 & 0.27457 & 0.000649 & 1 & 1 & 1 & 1 \\
\hline 1450 & $6 / 9 / 200911: 23$ & 0 & 3.1948 & 3.983 & 0 & 0.35219 & 0 & 1 & 1 & 1 & 1 \\
\hline 1450 & $6 / 9 / 200911: 23$ & 0 & 3.1948 & 3.8319 & 0.075531 & 0.16811 & 0 & 1 & 1 & 1 & 1 \\
\hline 1450 & $6 / 9 / 200911: 23$ & 0 & 3.1948 & 3.6736 & 0.089264 & 0.23243 & 0 & 1 & 1 & 1 & 1 \\
\hline 1450 & $6 / 9 / 200911: 23$ & 0 & 3.1948 & 3.669 & 0.09079 & 0.2413 & 0 & 1 & 1 & 1 & 1 \\
\hline 1450 & $6 / 9 / 200911: 23$ & 0 & 3.1948 & 3.7798 & 0 & 0.56954 & 0 & 1 & 1 & 1 & 1 \\
\hline 1450 & $6 / 9 / 200911: 23$ & 0 & 3.1948 & 3.9317 & 0 & 0.31671 & 0 & 1 & 1 & 1 & 1 \\
\hline 1450 & $6 / 9 / 200911: 23$ & 0 & 3.1948 & 3.9803 & 0 & 0.27457 & 0 & 1 & 1 & 1 & 1 \\
\hline 1450 & $6 / 9 / 200911: 23$ & 0 & 3.1948 & 3.885 & 0 & 0.31449 & 0 & 1 & 1 & 1 & 1 \\
\hline 1450 & $6 / 9 / 200911: 23$ & 0 & 3.1948 & 3.7376 & 0 & 0.34776 & 0 & 1 & 1 & 1 & 1 \\
\hline 1450 & $6 / 9 / 200911: 23$ & 0 & 3.1948 & 3.6497 & 0 & 0.29231 & 0 & 1 & 1 & 1 & 1 \\
\hline 1450 & $6 / 9 / 200911: 23$ & 0 & 3.1948 & 3.7248 & 0 & 0.31006 & 0 & 1 & 1 & 1 & 1 \\
\hline 1450 & $6 / 9 / 200911: 23$ & 0 & 3.1948 & 3.8727 & 0.043488 & 0.18142 & 0 & 1 & 1 & 1 & 1 \\
\hline 1450 & $6 / 9 / 200911: 23$ & 0 & 3.1948 & 3.9862 & 0 & 0.19029 & 0 & 1 & 1 & 1 & 1 \\
\hline 1450 & $6 / 9 / 200911: 23$ & 0 & 3.1948 & 3.94 & 0 & 0.27457 & 0 & 1 & 1 & 1 & 1 \\
\hline 1450 & $6 / 9 / 200911: 23$ & 0 & 3.1948 & 3.8118 & 0.031281 & 0.34332 & 0 & 1 & 1 & 1 & 1 \\
\hline 1450 & $6 / 9 / 200911: 23$ & 0 & 3.1948 & 3.6356 & 0 & 0.29231 & 0 & 1 & 1 & 1 & 1 \\
\hline 1450 & $6 / 9 / 200911: 23$ & 0 & 3.1948 & 3.6456 & 0 & 0.30562 & 0 & 1 & 1 & 1 & 1 \\
\hline 1450 & $6 / 9 / 200911: 23$ & 0 & 3.1948 & 3.761 & 0 & 0.29231 & 0 & 1 & 1 & 1 & 1 \\
\hline 1450 & $6 / 9 / 200911: 23$ & 0 & 3.1948 & 3.951 & 0 & 0.29453 & 0 & 1 & 1 & 1 & 1 \\
\hline
\end{tabular}


Appendix B (Datasheets) 
WIRELESS MEASUREMENT SYSTEM

- $2.4 \mathrm{GHz}$ IEEE 802.15.4, Tiny Wireless Measurement System

- Designed Specifically for Deeply Embedded Sensor Networks

- 250 kbps, High Data Rate Radio

- Wireless Communications with Every Node as Router Capability

- Expansion Connector for Light, Temperature, RH, Barometric Pressure, Acceleration/Seismic, Acoustic, Magnetic and other Crossbow Sensor Boards

\section{Applications}

- Indoor Building Monitoring and Security

- Acoustic, Video, Vibration and Other High Speed Sensor Data

- Large Scale Sensor Networks (1000+ Points)

\section{ZigBee"Alliance}

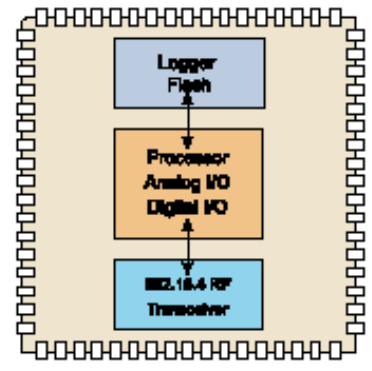

XM2110CA Block Diagran

\section{Crossbow}

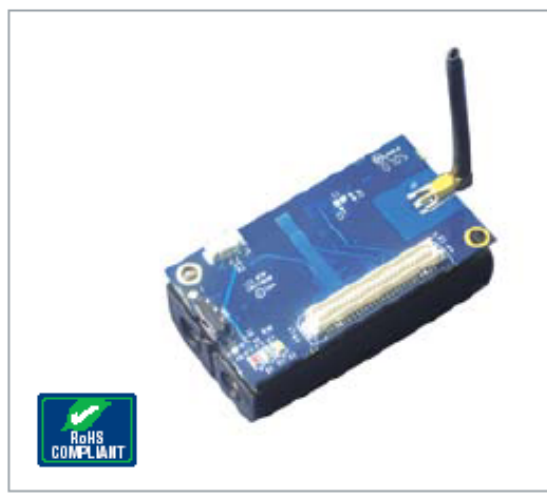

\section{IRIS}

The IRIS is a $2.4 \mathrm{GHz}$ Mote module used for enabling low-power, wireless sensor networks. The IRIS Mote features several new capabilities that enhance the overall functionality of Crossbow's wireless sensor networking products.

Product features Include:

- Up to three times improved radio range and twice the program memory over previous MICA Motes

- Outdoor line of-sight tests have yeilded ranges as far as 500 meters between nodes without amplification

- IEEE 802.15.4 compliant RF transceiver

- 2.4 to $2.48 \mathrm{GHz}$, a globally compatible ISM band

- Direct sequence spread spectrum radio which is resistant to $\mathrm{RF}$ interference and provides inherent data security

- 250 kbps data rate

- Supported by MoteWorks"' wireless sensor network platform for reliable, ad-hoc mesh networking

- Plug and play with Crossbow's sensor boards, data acquisition boards, gateways, and software

MoteWorks" enables the development of custom sensor applications and is specifically optimized for low-power, battery-operated networks. MoteWorks is based on the open-source TinyOS operating system and provides reliable, ad-hoc mesh networking, over-theair-programming capabilities, cross development tools, server middleware for enterprise network integration and client user interface for analysis and configuration.

\section{Processor \& Radio}

\section{Platform}

The XM2110CA is based on the Atmel ATmega1281. The ATmega 1281 is a low-power microcontroller which runs MoteWorks from its internal flash memory. A single processor board (XM2110) can be configured to run your sensor application/processing and the network/radio communications stack simultaneously. The IRIS 51-pin expansion connector supports Analog Inputs, Digital VO, 12C, SPI and UART interfaces. These interfaces make it easy to connect to a wide variety of external peripherals.

\section{Sensor Boards}

Crossbow offers a variety of sensor and data acquisition boards for the IRIS Mote. All of these boards connect to the IRIS via the standard 51-pin expansion connector. Custom sensor and data acquisition boards are also available. Please contact Crossbow for additional information. 


\begin{tabular}{|c|c|c|}
\hline Processor/Radio Board & XM2110CA & Remarks \\
\hline \multicolumn{3}{|l|}{ Processor Performance } \\
\hline Program Flash Memory & $128 K$ bytes & \\
\hline Measurement (Serial) Flash & $512 \mathrm{~K}$ bytes & > 100,000 Measurements \\
\hline RAM & SK bytes & \\
\hline Configuration EEPROM & 4K bytes & \\
\hline Serial Communications & UART & 0-3V transmission levels \\
\hline Analog to Digital Corverter & 10 bit $\mathrm{ADC}$ & 8 channal, $0-3 \mathrm{~V}$ input \\
\hline Other Interfaces & Digital $1 / 0,12 \mathrm{C}, 5 \mathrm{PP}$ & \\
\hline \multirow[t]{2}{*}{ Current Draw } & $8 \mathrm{~mA}$ & Active mode \\
\hline & $\delta \mu \mathrm{A}$ & Sleep mode (total) \\
\hline \multicolumn{3}{|l|}{ RF Transcelver } \\
\hline Frequency band & $2405 \mathrm{MHz}$ to $2420 \mathrm{MHz}$ & ISM band, programmable in $1 \mathrm{MHz}$ steps \\
\hline Transmit $\langle T X\rangle$ data rate & $250 \mathrm{kbps}$ & \\
\hline RF power & $3 \mathrm{dBm}$ (typ) & \\
\hline Raceive Sensitivity & $-101 \mathrm{dBm}(\mathrm{typ})$ & \\
\hline \multirow[t]{2}{*}{ Adjacent channel rejection } & $36 \mathrm{~dB}$ & + $5 \mathrm{MHz}$ chamel spacing \\
\hline & $34 \mathrm{~dB}$ & $-5 \mathrm{MHz}$ channel spacing \\
\hline Outdoor Range & $>300 \mathrm{~m}$ & $1 / 4$ wave dipole antenna, LOS \\
\hline Indoor Range & $>50 \mathrm{~m}$ & 1/4 wave dipole antenna, LOS \\
\hline \multirow[t]{4}{*}{ Current Draw } & $16 \mathrm{~mA}$ & Receive mode \\
\hline & $10 \mathrm{~mA}$ & $\mathrm{TX},-17 \mathrm{dBm}$ \\
\hline & $13 \mathrm{ma}$ & $\mathrm{TX},-3 \mathrm{dBm}$ \\
\hline & $17 \mathrm{~mA}$ & $\mathrm{TX}, 3 \mathrm{dBm}$ \\
\hline \multicolumn{3}{|l|}{ Electromechanical } \\
\hline Battery & 2XAA batteries & Attached pack \\
\hline External Power & $2.7 \mathrm{~V}-3.3 \mathrm{~V}$ & Molex comector provided \\
\hline User Interface & 3 LEDs & Red, geen and yellow \\
\hline Size (in) & $2.25 \times 1.25 \times 0.25$ & Excluding battery pack \\
\hline$(m m)$ & $58 \times 32 \times 7$ & Excluding battery pack \\
\hline Weight (oz) & 0.7 & Excluding batteries \\
\hline 〈grams〉 & 18 & Excluding batteries \\
\hline Expansion Connector & 51-pin & All major VO signals \\
\hline
\end{tabular}

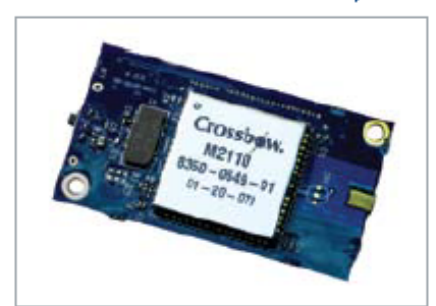

IRIS Mote (bottom view)

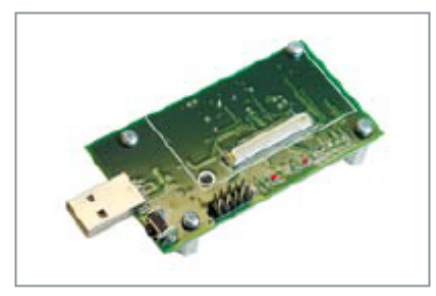

MIB520CA Mote Interface Board

Base Stations

A base station allows the aggregation of sensor network data onto a PC or other computer platform. Any IRIS Mote can function as a base station when it is connected to a standard PC interface or gateway board. The MIB510 or MIB520 provides a serial/USB interface for both programming and data communications. Crossbow also offers a stand-alone gateway solution, the MIB600 for TCP/IP-based Ethernet networks.

Ordering Information

\begin{tabular}{|l|l|}
\hline Model & Description \\
\hline XM2110CA & 2.4 GHz IRIS OEM Reference Board \\
\hline
\end{tabular}

Document Part Number: 6020-0124-01 Rev A 


\section{Crossb}

\section{MTS420/400 \\ ENVIRONMENTAL SENSOR BOARD}

- Compatible with IRIS/MICAz/ MICA2 Processor/ Radio Boards

- Onboard Temperature \& Humidity, Barometric Pressure and Ambient Light Sensors

- Dual-Axis Accelerometer

- 64K EEPROM (CC versions), 2K EEPROM (CB versions) for User Configuration Data

- Optional GPS Module

Applications

- Agricultural Monitoring

- Art Preservation

- Environmental Monitoring

- Sensor Location Mapping* (*GPS Equipped)

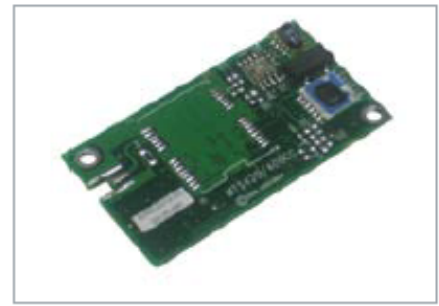

MTS400CC

\section{MTS420/400}

Developed in conjunction with UC Berkeley and Intel Research Labs, the MTS400 and MTS420 offer five basic environmental sensing parameters and an optional GPS module (MTS420).

These sensor boards utilize the latest generation of IC-based surface mount sensors. These energy-efficient digital devices in turn provide extended battery-life and performance wherever low maintenance field-deployed sensor nodes are required.

These versatile sensor boards are intended for a wide variety of applications ranging from a simple wireless weather station to a full mesh network of environmental monitoring nodes. Applicable industries include Agricultural, Industrial, Forestry, HVAC and more.

\section{Specifications:}

\section{Board}

- Operating temp. range: $-10^{\circ} \mathrm{C}$ to $+60^{\circ} \mathrm{C}$

- Operating humidity range $0 \% \mathrm{RH}$ to $90 \%$ RH non-condensing

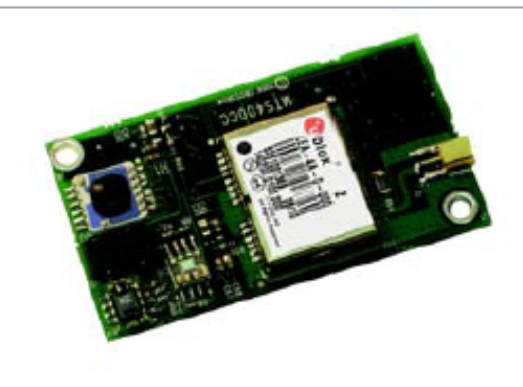

MTS420CC (with GPS Module)

Dual-axis Accelerometer

- Analog Devices ADXL202JE

- Acceleration range; resolution: $\pm 2 \mathrm{~g} ; 2 \mathrm{mg}$ at $60 \mathrm{~Hz}$

- Nonlinearity: $0.2 \%$ of full scale

- Zero g bias level: $2.0 \mathrm{mg} /{ }^{\circ} \mathrm{C}$ from $25^{\circ} \mathrm{C}$

Barometric Pressure Sensor

- Intersema MS5534AM

- Pressure range; resolution: 300-1100 mbar; $0.01 \mathrm{mbar}$

- Accuracy: $\pm 1.5 \%$ at $25^{\circ} \mathrm{C}$

Ambient Light Sensor

- TAOS TSL2550D

- Spectral responsivity: 400-1000 nm, similar to human eye

Relative Humidity \& Temperature Sensor

- Sensirion SHT11

- Humidity range; resolution: 0-100\% $\mathrm{RH} ; 0.03 \% \mathrm{RH}$

- Absolute RH accuracy: $\pm 3.5 \% \mathrm{RH}$

- Temp. accuracy: $\pm 0.5^{\circ} \mathrm{C} @ 25^{\circ} \mathrm{C}$

GPS Module (MTS420CC only)

- ublox LEA-4A

- Tracking channels: 16

- L1 frequency: $1575.42 \mathrm{MHz}$ C/A code

- Position accuracy: $10 \mathrm{~m}, 2 \mathrm{D}$

- Reacquisition time: < 1 sec. (typ.); (<30 sec. max. blockage)

Ordering Information

\begin{tabular}{|l|l|}
\hline Model & Description \\
\hline MTS400CC & Weather Sensor Board with Light, Temperature, Humidity, Barometric Pressure and Seismic \\
\hline MTS420CC & Weather Sensor Board with Light, Temperature, Humidity, Barometric Pressure, Seismic and GPS \\
\hline
\end{tabular}

Document Part Number: 6020-0053-04 Rev A 


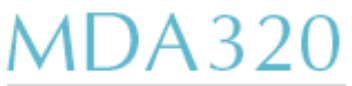

DATA ACQUISITION BOARD

- Multi-Function Data Acquisition Board

- Compatible with MoteView Driver Support

- Up to 8 Channels of 16 -bit Analog Input

- Onboard Sensor Excitation and High-Speed Counter

- Convenient Micro-Terminal Screw Connections

\section{Applications}

- Industrial Vibration Monitoring

- Seismic and Acoustic Analysis

- Controlled Environment Agriculture

- HVAC Instrumentation and Control

- Environmental Data Collection

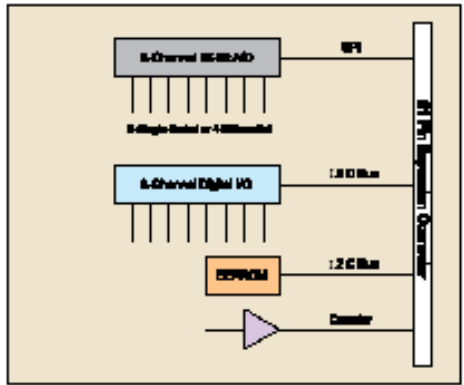

MDA320CA Block Diagram

\section{Crossbew}

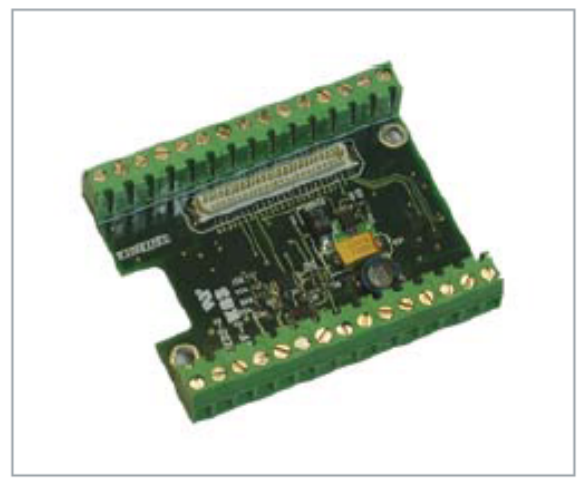

\section{MDA320CA}

The MDA320CA is a high-performance data acquisition board with up to 8 channels of 16-bit analog input. It combines a reduced feature set with the same versatile functionality found in Crossbow's popular MDA300 data acquisition board. This board is designed for use in cost-sensitive applications requiring precision data collection and analysis.

With improved micro-terminal connections, the MDA320CA offers users a rapid and convenient interface to a wide variety of discrete external sensing devices. Data logging and display is supported via Crossbow's MoteView user interface.

Crossbow's MoteView software is designed to be the primary interface between a user and a deployed network of wireless sensors. MoteView provides an intuitive user interface to database management along with sensor data visualization and analysis tools. Sensor data can be logged to a database residing on a host $\mathrm{PC}$, or to a database running autonomously on a Stargate gateway.
Ordering Information

\begin{tabular}{|l|l|}
\hline Model & Description \\
\hline MDA320CA & Mote Data Acquistion Board \\
\hline
\end{tabular}

Communication and control features Include:

- 8 single-ended $0-2.5 \mathrm{~V}$ inputs, or 4 differential $0-2.5 \mathrm{~V}$ ADC channels

- 8 digital $0-2.5 \mathrm{~V} \mathrm{V/O}$ channels with event detection interrupt

- 2.5, 3.3, $5 \mathrm{~V}$ battery sensor excitation and low-power mode

- 64K EEPROM for onboard sensor calibration data

- $200 \mathrm{~Hz}$ counter channel for wind speed, pulse frequencies

- External $I 2 C$ interface included in Crossbow's MoteWorks"w software platform. MoteWorks enables the development of custom sensor applications and is specifically optimized for low-power, batteryoperated networks. MoteWorks is based on the open-source TinyOS operating system and provides reliable, ad-hoc mesh networking, over-the-air-programming capabilities, cross development tools, server middleware for enterprise network integration and client user interface for analysis and configuration.
Drivers for the MDA300 board are 


\section{MIB520}

USB INTERFACE BOARD

- Base Station for Wireless Sensor Networks

- USB Port Programming for IRIS/MICAz/MICA2 Hardware Platforms

- Supports JTAG code debugging

- USB Bus Power

\section{Applications}

- USB Interface

- Testbed Deployments

- In-System Programming

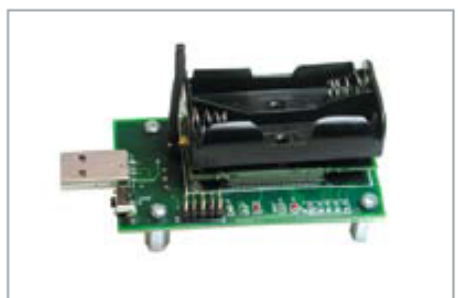

MIB520CB with attached Mote

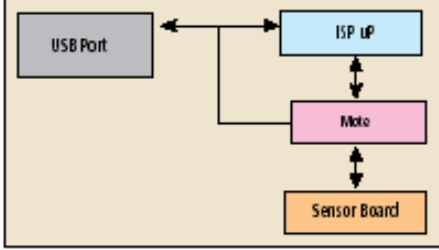

MIB520CB Block Diagram

\section{Crossb}

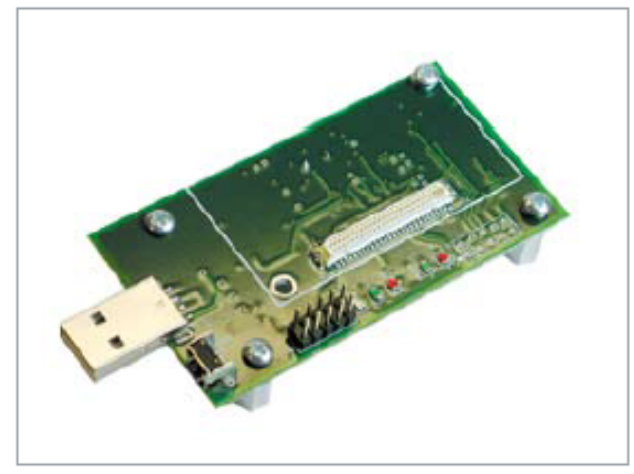

\section{MIB520CB}

The MIB520CB provides USB connectivity to the IRIS and MICA family of Motes for communication and in-system programming. Any IRIS/ MICAz/MICA2 node can function as a base station when mated to the MIB520CB USB interface board. In addition to data transfer, the MIB520CB also provides a USB programming interface.

The MIB520CB offers two separate ports: one dedicated to in-system Mote programming and a second for data communication over USB. The MIB520CB has an on-board processor that programs Mote Processor Radio Boards. USB Bus power eliminates the need for an external power source.
Specifications

USB Interface

- Baud Rate: $57.6 \mathrm{~K}$

- Male to Female USB cable (included with unit)

Mote Interface

- Connectors:

- 51-pin

- Indicators:

- Mote LED's: Red Green, Yellow

Programming Interface

- Indicators:

- LEDs - Power Ok (Green),

Programming in Progress (Red)

- Switch to reset the programming processor and Mote.

Jtag Interface

- Connector: 10-pin male header POWER

- USB Bus powered

\section{Ordering Information}

\begin{tabular}{|l|l|}
\hline Model & Description \\
\hline MiB520CB & USB PC Interface Board \\
\hline
\end{tabular}




\section{?}

\section{ce}

\section{Order Code}

\section{UC6000-FP-IUE2-R2-P5}

Features

- Analogue output, load-dependent voltage or current

- Switch output

- Serial interface

- Synchronisation options

- Temperature compensation

- Absolute polarity reversal protection

- Parameterisable with ULTRA 3000

\section{Electrical Connection}

Sta ndard symbolConnection:

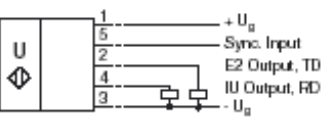
둔 IU Output, $F D$
Dimensions

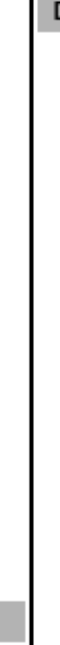
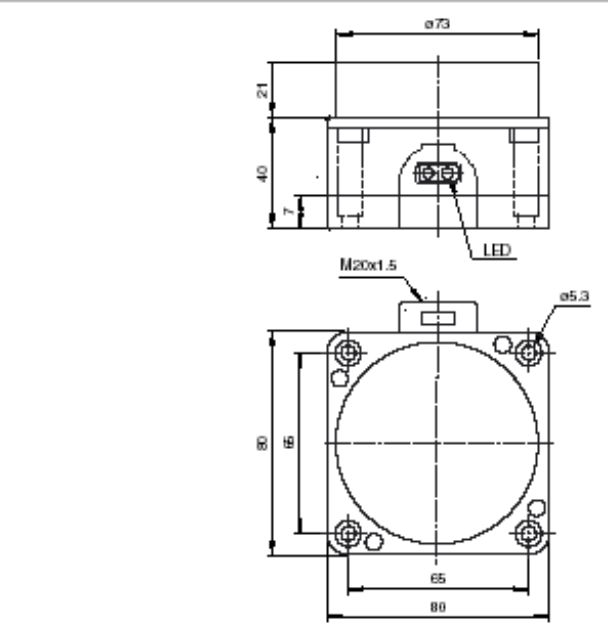

\section{Technical Data}

General specincations

Sensing range

Unusabla area

Standard target plate

Transduoes irequan
Presponse delay

$800 \ldots 6000 \mathrm{rrm}$

$0 . . .800 \mathrm{~mm}$
$100 \mathrm{~mm} \times 100 \mathrm{~mm}$

approc $65 \mathrm{kHz}$

bor factory sedting

minmal (EM; NCNE): $5190 \mathrm{~ms}$ (2 measurng cycles)

dynami (EM, DrN): $: 270 \mathrm{~ms}$ (3 meesuring cycles)

Indlcators/operatling means

LED yellow
LED redigeen

suitching state switch outpul

permanently green : "Power on", llashes durng standby operation

Electrical specifications

Cperating voltage red llashing: "Error", (e. g. backgound nokse level too highi)

No-bed supply current

$20 \ldots 30$ V DC, ripple $10 \%$

Interface

Interlace type

Input/output

Synctronisation

$560 \mathrm{ma}$

Pukse length
Pause kngth

Sanchonisatbon frequency

S)nctro

Cutput type

PS 232, 9600 bits, no peritly 8 data bits, 1 stop tut ( $\$ 10$ - CFF)

1 synctronous ocnnection, bitirectional

orged: $U_{B} \ldots\left(-U_{B}+1 V\right)$, 1-level: $\left(-U_{B}+5 V\right) \ldots+U_{B}$ $\geq 100 \mu \mathrm{s}$

$\leq 10 \mathrm{~Hz}$, with exdarnal synchrentsation

1 smitch culput E5: prp NONC smitchable

1 anakgue culput, load dependent:

$\mathrm{R}_{\mathrm{L}} \leq 500 \mathrm{Chm}$ : curent output $4 \ldots .20 \mathrm{~mA}$

$R_{L} \geq 1$ kChm: veltage oulput $2 \ldots 10 \mathrm{~V}$

$\begin{array}{ll}\text { Presolution } & \text { depending on the set eraluatlon range: } \\ & 0.172 \mathrm{~mm} \text {, if evaluatlon range }<706 \mathrm{~mm}\end{array}$

Devation o the charackeristlc exaluation range [mm] / 3296, when evaluation range > $705 \mathrm{~mm}$

$\leq 0.2 \%$

Proegt accuacy $\quad \leq 0.1 \%$ of tul-scale ralue

$\begin{array}{ll}\text { Pepeat accuacy } & \leq 0.1 \% \text { of tul-scale value } \\ \text { Pated cperatbral current } I_{0} & 200 \mathrm{~mA} \text {, short-drcut tonsload protected }\end{array}$

valtage drop $U_{\text {. }}$

$\leq 3 \vee \mathrm{DC}$

Range hysteresis H $\quad \leq 1 \%$ of the set operating distance

Temperature inilluenos

Standards

Amblent condittions

Ambient temperatue

Sibrage temperature
Mechanlcal specifications

Probctiondegree

Connectlon

Materlal

Housing

Transivoses $2 \%$

EN $80047-5-2$

$-25 \ldots 70 \mathrm{O} C(248 \ldots 343 \mathrm{~K})$
$-40 \ldots 50^{\circ} \mathrm{C}(233 \ldots 359 \mathrm{~K})$

IP65

terminal compartment, $\leqslant 2.5 \mathrm{mmm}^{2}$ conductor cs

PBT

epoxy resinhollow glass sphere mixture; pdyurethane bam 
Description of the sensor functions

The outputs of the sensor can be used in two different operating modes: Switching/ analogue mode, or RS 232 mode (RS 232, $9600, n, 8,1$ ). Select the operating mode with DIP switch 10. The limits of the IU ramp are set with the DIP switches 14 and 5-8 (see table). Switch 9 is used to set the close or open function of the switch output.

For further information on the sensor's command set, please see the publication "Command Set for Ultrasonic Sensors with RS 232 Interface".

Caution: Ensure that DIP switch S10 is correctly set before connecting the RS 232 interface.

Synchronisation

The sensor features a synchronisation input for the suppression of mutual interferenœ. If this input is not used, the sensor will operate using an intemally generated clock rate. It can be synchronised by applying a square wave voltage. A falling edge leads to the transmission of a single ultrasonic pulse. A low level $>1 \mathrm{~s}$ or an open synchronisation input will result in the nomal operation of the sensor. A high level $>1 \mathrm{~s}$ will result in the standby operation of the sensor (grøen LED).

Several functions are available:

- Two to tive sensors can be synchronised by interconnecting their synchronisation inputs. In this case, the sensors alternately transmitultrasonic pulses.

- Multiple sensors can be controlled by the same synchronisation signal. The sensors are synchronised.

- The synchronisation pulses are sent cyclically to individual sersors. The sensors operate in multiplex mode.

The response time increases when sensors are synchronised as the measuring cycle time is increased by the synchronisation.

Adjustment of the evaluation window via coding switch in terminal compartment

\begin{tabular}{|c|c|c|c|}
\hline \begin{tabular}{|l|} 
Switch \\
1234 \\
\end{tabular} & \begin{tabular}{|l|}
$\mathrm{NDE}$ \\
{$[\mathrm{mm}]$}
\end{tabular} & \begin{tabular}{|l|} 
Switch \\
5678 \\
\end{tabular} & \begin{tabular}{|l}
$\begin{array}{l}F D E \\
{[\mathrm{~mm}]}\end{array}$ \\
\end{tabular} \\
\hline 0000 & 800 & 0000 & 800 \\
\hline 0001 & 1100 & 0001 & 1100 \\
\hline 0010 & 1400 & 0010 & 1400 \\
\hline 0011 & 1700 & 0011 & 1700 \\
\hline 0100 & 2000 & 0100 & 2000 \\
\hline 0101 & 2300 & 0101 & 2300 \\
\hline 0110 & 2650 & 0110 & 2650 \\
\hline 0111 & 3000 & 0111 & 3000 \\
\hline 1000 & 3350 & 1000 & 3350 \\
\hline 1001 & 3700 & 1001 & 3700 \\
\hline 1010 & 4050 & 1010 & 4050 \\
\hline 1011 & 4400 & 1011 & 4400 \\
\hline 1100 & 4800 & 1100 & 4800 \\
\hline 1101 & 5200 & $\begin{array}{llllllll}1 & 1 & 1\end{array}$ & 5200 \\
\hline 1110 & 5600 & 1110 & 5600 \\
\hline 1111 & 6000 & 1111 & 6000 \\
\hline
\end{tabular}

DIP Switches in Terminal Compartment: Adjustment of the Target Window

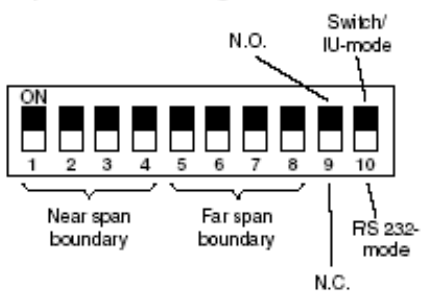

rear span bandary < dietant limt $\rightarrow$ ULrising shpe

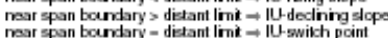
Switch point swtch outpu: (NDE + FDEY2 (Precorfigration)
Characteristic Curves/Additional Information

\section{Characteristic response curves}

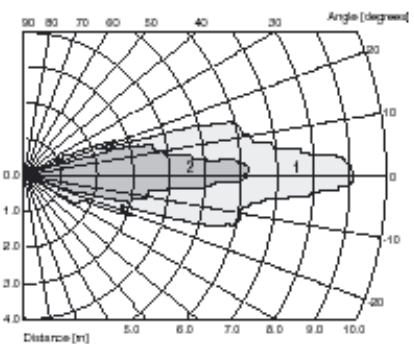

Curve 1: fht surbaco $100 \mathrm{~mm} \times 100 \mathrm{~mm}$
Curve 2: rand bar, $026 \mathrm{~mm}$

\section{LED-Window}

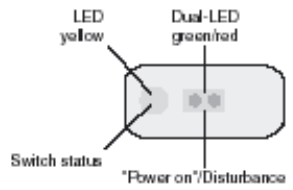

Accessories

MH 04-3505

Mounting aid

MHW 11

Mounting aid

PAO2

Acoessories

ULTRA3000

Soltware for ultrascnic sensors, ocmfort line

UC-FP/U9-R2

Acoessories 
Thanks to its extensive command set, the sensor can be configured to suit the application via the RS 232 interface.

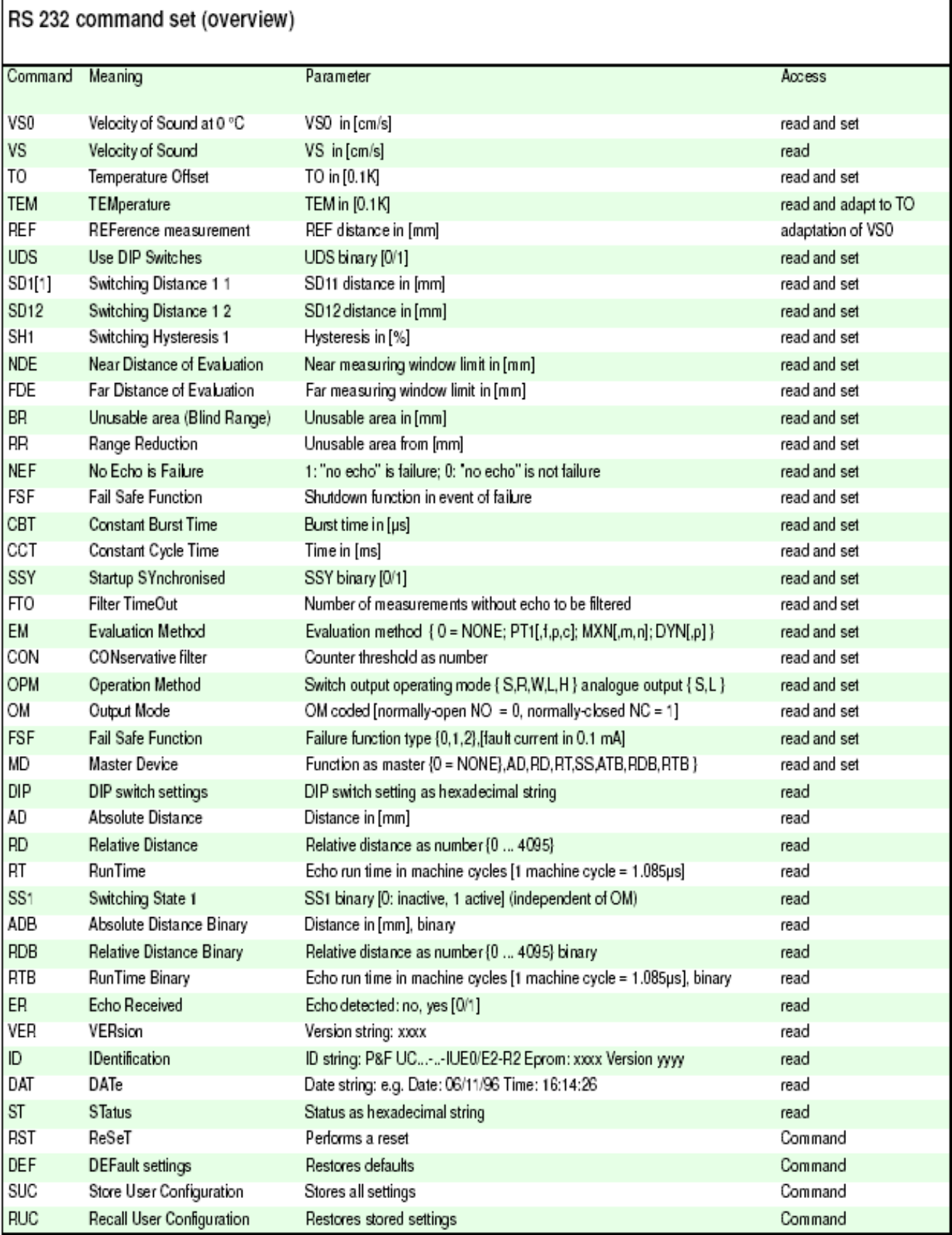

Programming instructions

Caution: When programming the sensor via the integrated RS 232 interface, ensure that DIP switch 10 is in the OFF (RS 232 mode) position before connecting the interface cable.

Electrical connection of interface cable UC-FP/U9-R2 (see aocessories).

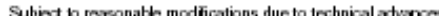




\begin{tabular}{|c|c|}
\hline $\begin{array}{c}\text { Interface cable } \\
\text { Conductor oobur }\end{array}$ & $\begin{array}{c}\text { Sensor terminal compartment } \\
\text { Terminal no. }\end{array}$ \\
\hline brown (TD) & $4(\mathrm{RD})$ \\
\hline black (RD) & $2(\mathrm{TD})$ \\
\hline blue (GND) & $3\left(-\mathrm{U}_{\mathrm{B}}\right)$ \\
\hline
\end{tabular}

Structure of the filter functions

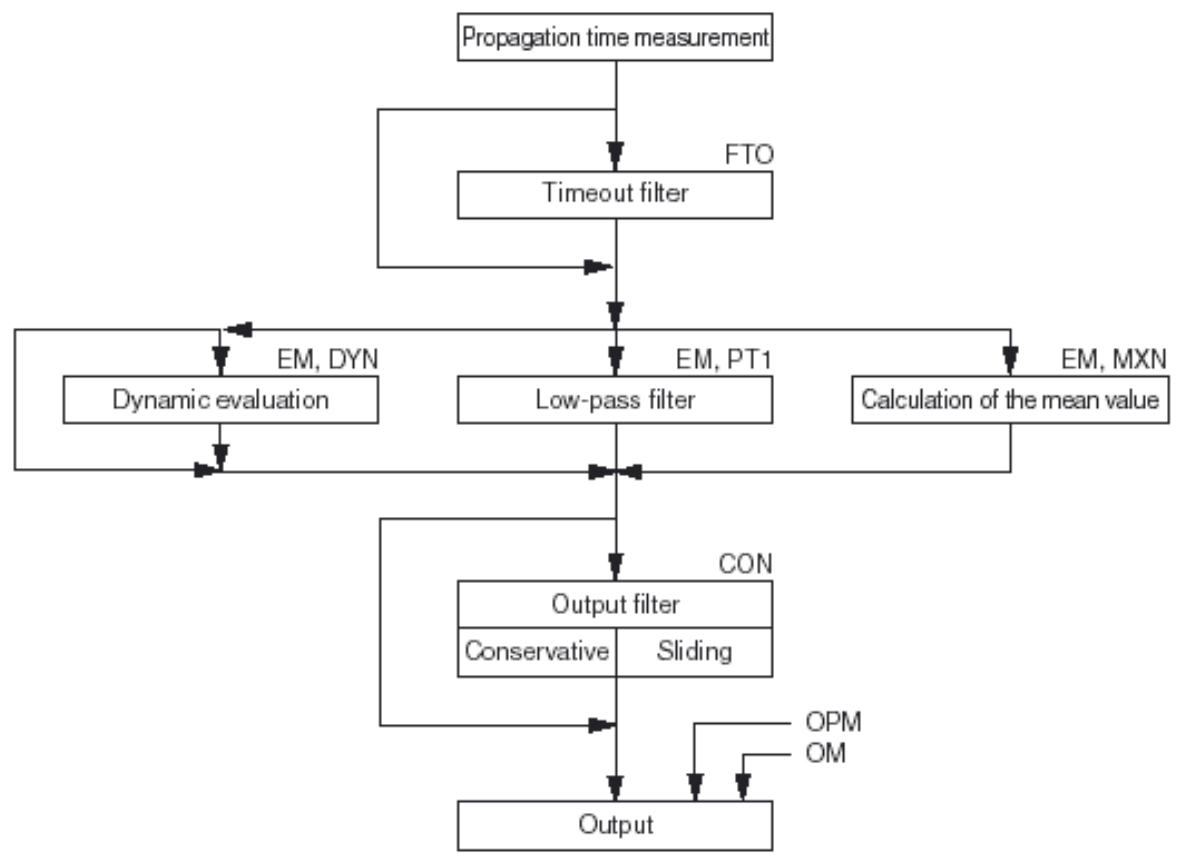




\section{AST4500 \& AST 4510 class 1 Div 1, Groups $C$ and D with Approved Barrier}

Submersible Stainless Steel Media Isolated Pressure Sensor

Overview

The AST4500 and AST4510 submersible pressure sensors are approved to UL/CUL913 (CSA 157) Class 1 Div 1, Groups C and D for use in intrinsically safe areas with an approved barrier. For pressure ranges from $0-2.5$ to 0-100 PSI that require a wide range of media compatibility, the submersible series is an excellent solution to level monitoring for indoor and outdoor applications.

\section{Benefits}

- High Strength Stainless Steel Construction

- No Internal O-rings

- Wide Operating Temperature Range

- Ranges up to 100 PSI

- Low Static and Thermal Errors

- Unparalleled Price and Performance

- Rugged Design Survives Harsh Environments

- Compatible with Wide Range of Liquids

- EMI/RFI Protection

\section{Applications}

- Ground Water Level Depth Measurement

- Earthen $\&$ Concrete Dams

- Liquid Tanks

- Gasoline \& Diesel Fuel Tanks

- Irrigation

- Waste Water Canals

\begin{tabular}{|ll|}
\hline Performance (1) $\mathbf{2 5}^{\circ} \mathbf{C ~ ( 7 7 ^ { \circ } \mathbf { F } )}$ \\
\hline Accuracy* & $< \pm 0.25 \% \mathrm{BFSL}$ \\
Stability (1 year) & $\pm 0.25 \% \mathrm{FS}$, typical \\
Over range Protection & $2 X$ Rated Pressure \\
Burst Pressure & $5 X$ or $1,250 \mathrm{PSI}$ (whichever is less) \\
Pressure Cycles & $>50$ Million \\
\hline -Accuracy indudes non-linearity, hysteresis \& non-repeatability \\
\hline
\end{tabular}

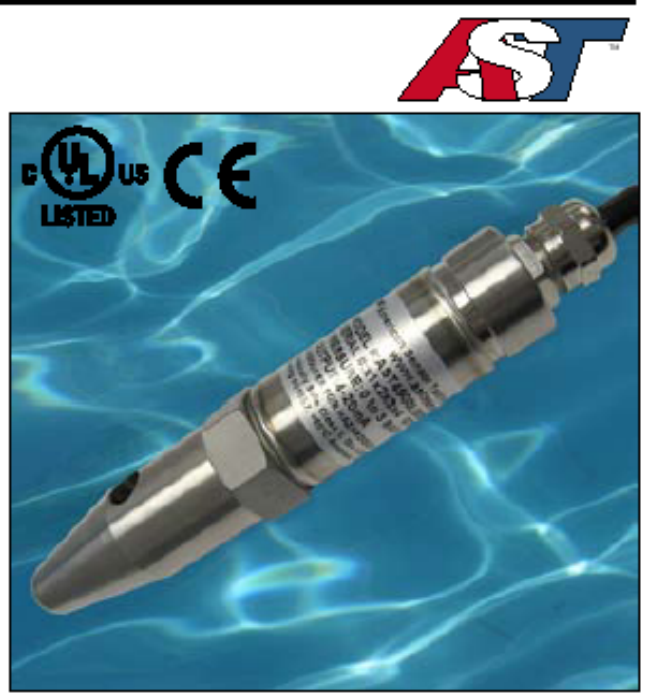

\begin{tabular}{|ll|}
\hline Environmental Data \\
\hline $\begin{array}{l}\text { Temperature } \\
\text { Operating }\end{array}$ & -40 to $85^{\circ} \mathrm{C}\left(-40\right.$ to $\left.185^{\circ} \mathrm{F}\right)$ \\
Storage & -40 to $100^{\circ} \mathrm{C}\left(-40\right.$ to $\left.212^{\circ} \mathrm{F}\right)$ \\
Thermal Limits & \\
Compensated Range & 0 to $55^{\circ} \mathrm{C}\left(30\right.$ to $\left.130^{\circ} \mathrm{F}\right)$ \\
TC Zero & $< \pm 1.5 \%$ of $\mathrm{FS}$ \\
TC Span & $< \pm 1.5 \%$ of $\mathrm{FS}$ \\
Other & \\
Shock & $100 \mathrm{G}, 11 \mathrm{msec}, 1 / 2$ sine \\
Vibration & $10 \mathrm{G}$ peak, 20 to $2000 \mathrm{~Hz}$. \\
EMliRFI Protection & Yes \\
Rating & $\mathrm{IP}-68$ \\
\hline
\end{tabular}

\begin{tabular}{|c|c|c|}
\hline \multicolumn{3}{|l|}{ Electrical Data } \\
\hline Output & $4-20 \mathrm{~mA}$ & $1.5 \mathrm{VDC}$ \\
\hline Excitation & $10-28 \mathrm{VDC}$ & 10-28VDC \\
\hline Output Impedance & $>10 \mathrm{k}$ Ohms & $<100$ Ohms, Nominal \\
\hline Current Consumption & $20 \mathrm{~mA}$, typical & $<10 \mathrm{~mA}$ \\
\hline Bandwidth & $(-3 \mathrm{~dB}): \mathrm{DC}$ to $250 \mathrm{~Hz}$ & $(-3 \mathrm{~dB}): \mathrm{DC}$ to $1 \mathrm{kHz}$ \\
\hline Output Noise & - & $<2 m V R M S$ \\
\hline Zero Offset & $< \pm 1 \%$ of FS & $< \pm 1 \%$ of $F S$ \\
\hline Span Tolerance & $< \pm 2 \%$ of FS & $< \pm 1.5 \%$ of FS \\
\hline Output Load & 0-800 Ohms@10-28VDC & $10 \mathrm{k}$ Ohms, min \\
\hline Reverse Polarity Protection & Yes & Yes \\
\hline
\end{tabular}

American Sensor Technologies . 450 Clark Dr., Mt. Olive, NJ 07828 · phone (973) 448-1901 · fax (973) 448-1905 · email: info@astsensors.com 


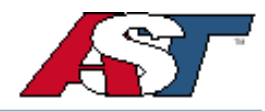

\section{Ordering Information}

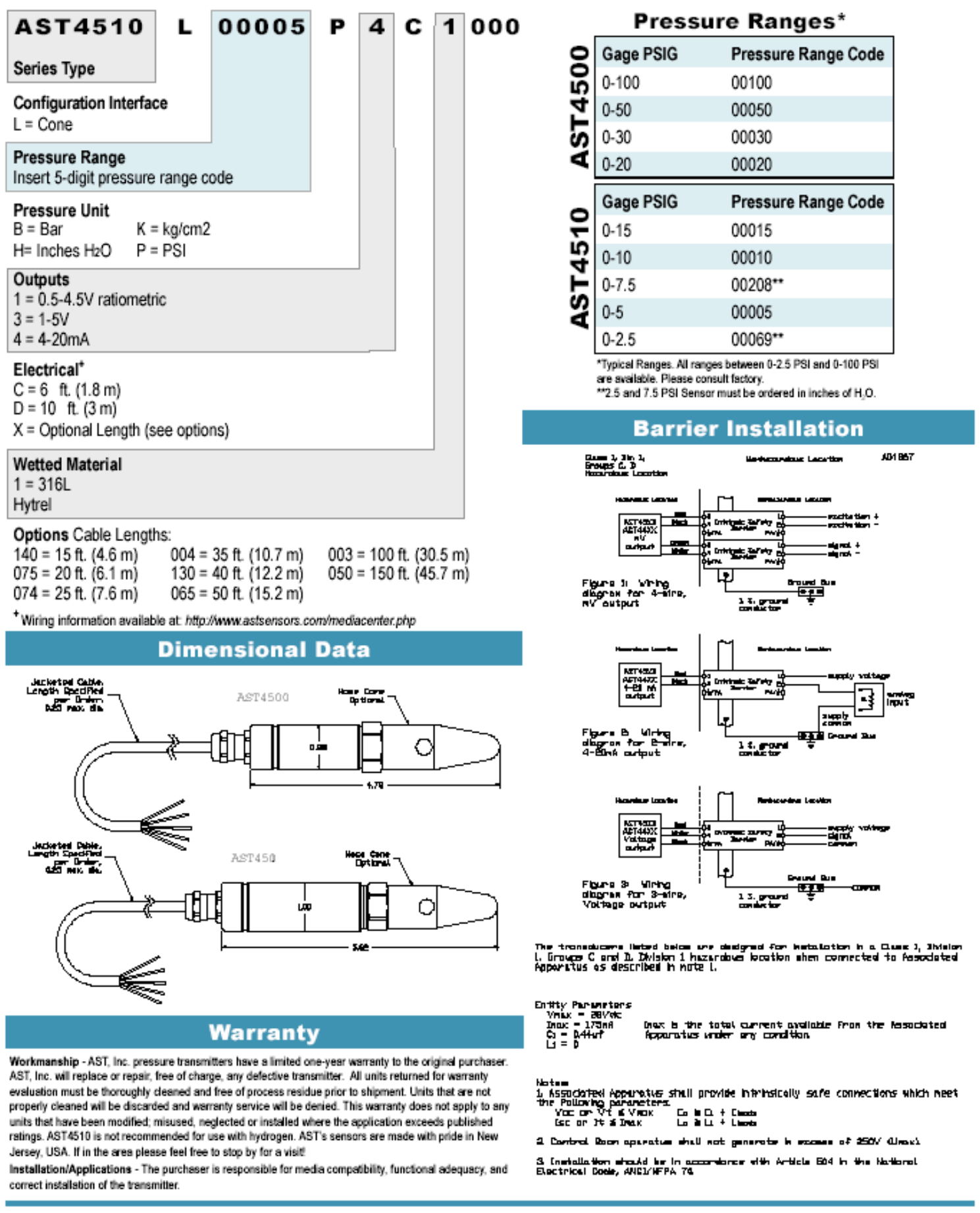




\section{PA series \\ RATIOMETRIC VOLTAGE OUTPUT}

Analog absolute positioning

Utilizing a precision potentiometer, the UniMeasure PA series position transducer provides basic absolute positioning with an analog output. With a steady state input voltage and with the potentiometer connected as a voltage divider, the ratiometric output voltage is directly proportional to wire rope extension. The

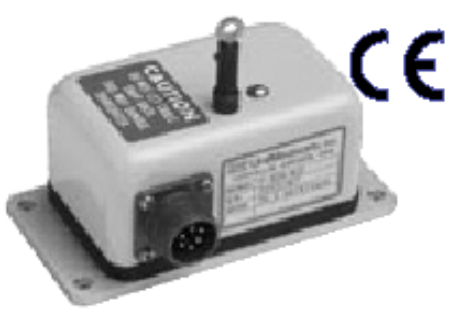
unit will function with any input voltage up to 25 volts maximum. To obtain best output linearity, the input voltage should be well regulated.

\begin{tabular}{|c|c|}
\hline 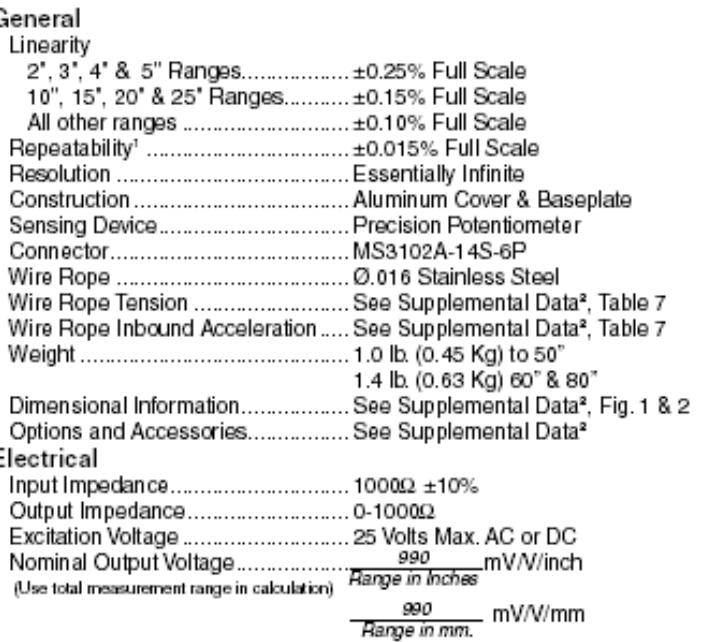 & 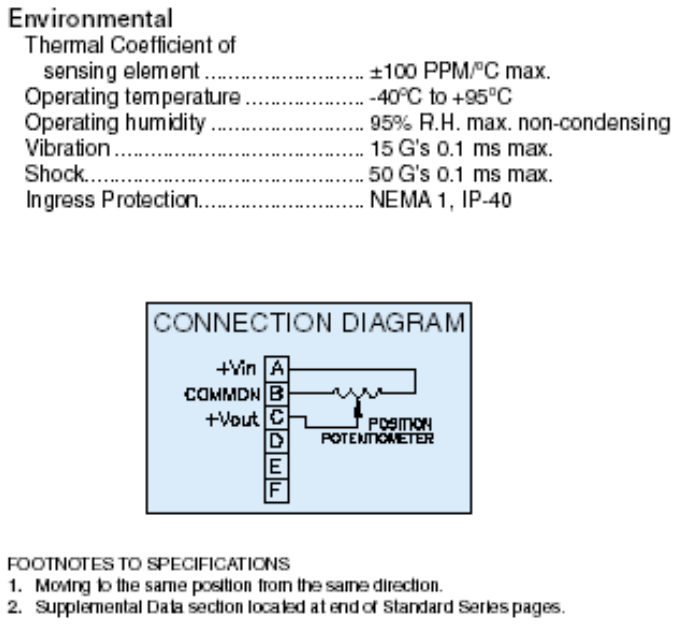 \\
\hline
\end{tabular}

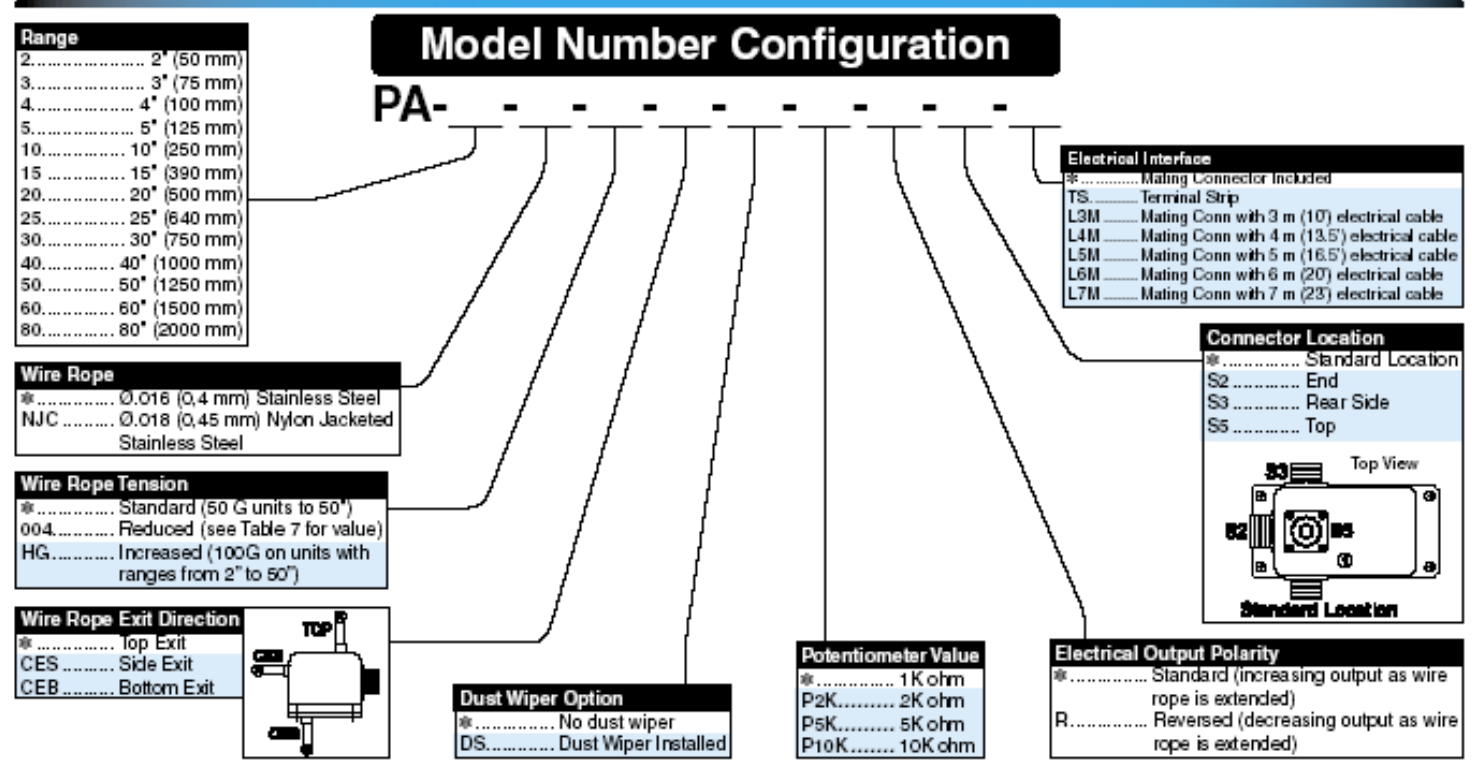

NOTE
1) *-Asterisk items are standard configuration. No option designator is required.

2) Shaded options available at additional cost.

3) See Supplemental Data for options.

Example

PA-50-NJC-DS-L3M

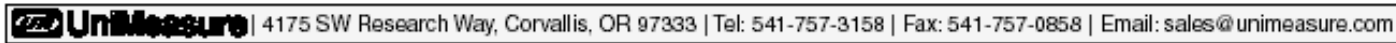




\section{STANDARD SERIES SUPPLEMENTAL DATA}

ADDITIONAL OPTIONS

\begin{tabular}{|c|c|c|}
\hline OPTION & $\begin{array}{c}\text { OPTION } \\
\text { DESIGNATOR }\end{array}$ & DESCRIPTION \\
\hline Nylon jacketed wire rope & NJC & $\begin{array}{l}\text { Replaces standard stainless steel wire rope with } \varnothing .018 \text { nylon jacketed } \\
\text { wire rope. Increases wire rope life dramatically but may increase non- } \\
\text { linearity by as much as } \pm 0.05 \% \text { of full scale. }\end{array}$ \\
\hline Reduced Wire Rope Tension & 004 & $\begin{array}{l}\text { Reduces the overall tension in the wire rope and increases wire rope } \\
\text { life. Dynamic response of the transducer is reduced due to the reduced } \\
\text { inbound acceleration capability. }\end{array}$ \\
\hline Increased Wire Rope Tension & $H G$ & $\begin{array}{l}\text { Increases tension in the wire rope which increases the dynamic } \\
\text { response of the transducer. On selected units with range of } 50 \text { " (1250 } \\
\text { mm) or less, inbound acceleration capability is } 100 \mathrm{G} \text { 's. Wire rope life } \\
\text { may be adversely affected by the HG option. }\end{array}$ \\
\hline Terminal strip & TS & Replaces connector with a terminal strip. \\
\hline Reversed output & $\mathbf{R}$ & $\begin{array}{l}\text { Output is at a maximum when wire rope is fully retracted. Output } \\
\text { decreases as wire rope is extended. Does not apply to velocity signal. }\end{array}$ \\
\hline $\begin{array}{l}\text { Non-standard potentiometer } \\
\text { (applies to PA series only) }\end{array}$ & PXK & $\begin{array}{l}\text { Replace ' } \mathrm{X} \text { " in option designator with required potentiometer value in K } \\
\text { ohms. Non-standard potentiometer linearity is as follows: } \\
\text { Ranges } 0 \text { to } 2 \text { ' to } 0 \text { to } 5 \text { "......... } \pm 1.00 \% \text { of full scale } \\
\text { Ranges } 0 \text { to } 10 \text { ' to } 0 \text { to } 25 \text { '...... } \pm 0.50 \% \text { of full scale } \\
\text { Ranges } 30 \text { " and above..................... } \pm 0.25 \% \text { of full scale } \\
\text { Note: This option is subject to potentiometer availability. }\end{array}$ \\
\hline
\end{tabular}

\section{ACCESSORIES}

\section{Auxiliary Wire Rope Extension Kit}

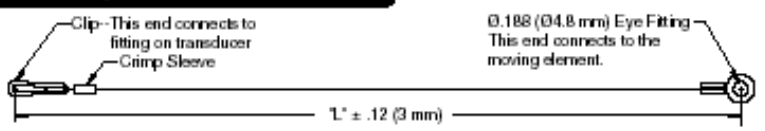

The auxiliary wire rope extension may be used to facilitate mounting the transducer remotely from the measurement point. The clip on the extension attaches to the eye fitting on the transducer. The eye fitting on the opposite end which is identical to the fitting on the transducer mounts to the moving element. The extension kit is also available with the clip end unterminated for situations where it is more convenient to size the wire rope length during installation. The clip and crimp sleeve are included as loose parts for user termination.

\begin{tabular}{|l|l|l|}
\hline Dimension "L" \\
Specify dimension " $\mathrm{L}$ " in centimeters to the nearest whole centimeter. \\
Note 1. $1 \mathrm{~cm}=0.394^{\prime}, 1$ inch $=2.54 \mathrm{~cm}$ \\
Note 2. Shortest length " $\mathrm{L}$ ' is $5 \mathrm{~cm}$ (approximately 2).
\end{tabular}

\section{Replacement Wire Rope Kit}

The replacement Wire Rope Kit includes a new wire rope with all end terminations, wire rope guide, felt dust wiper where applicable and installation instructions. To order, replace ' $x x^{\prime}$ in the part number with the applicable measurement range in inches.

10107-xx Replacement Wire Rope Kit_Standard $\varnothing .016^{\circ}$ stainless steel wire rope.

10108-xx Replacement Wire Rope Kit NJC option, $\varnothing .018^{\circ}$ nylon jacketed stainless steel wire rope.

10127-xx Replacement Wire Rope Kit DS option, Standard Ø.016" stainless stœel wire rope with dust wiper.

10128-xx Replacement Wire Rope Kit NJC and DS options, $\varnothing .018^{\circ}$ nylon jadketed stainless steel wire rope with dust wiper.

T- Uni 4175 SW Research Way, Corvallis, OR 97333 | Tel: 541-757-3158 | Fax: 541-757-0858 | Email: sales@ unimeasure.com 


\section{STANDARD SERIES \\ SUPPLEMENTAL DATA}

ADDITIONAL SPECIFICATIONS

\section{TABLE 7}

\section{PA, PB, P420, P510, P1010 SERIES}

\begin{tabular}{|c|c|c|c|c|c|c|c|c|c|c|c|c|c|}
\hline $\begin{array}{c}\text { Range } \\
\text { Designator }\end{array}$ & \multicolumn{2}{|c|}{ Range } & \multicolumn{2}{|c|}{$\begin{array}{l}\text { Wire Rope } \\
\text { Standard } \\
\text { Tension } \\
\text { (Nominal) }\end{array}$} & $\begin{array}{l}\text { Wire Rope } \\
\text { Standard } \\
\text { Acceleration } \\
\text { (G's) }\end{array}$ & \multicolumn{2}{|c|}{$\begin{array}{l}\text { Wire Rope } \\
\text { Reduced } \\
\text { Tension } \\
\text { (004 option) }\end{array}$} & $\begin{array}{c}\text { Wire Rope } \\
\text { Reduced } \\
\text { Acceleration } \\
\text { (004 option) } \\
\text { (G's) }\end{array}$ & $\begin{array}{l}\text { Wire Rope } \\
\text { Reduced } \\
\text { Tension } \\
\text { (004 option) }\end{array}$ & $\begin{array}{c}\text { Wire Rope } \\
\text { Reduced } \\
\text { Acceleration } \\
\text { (004 option) } \\
\text { (G's) }\end{array}$ & \multicolumn{2}{|c|}{$\begin{array}{l}\text { Wire Rope } \\
\text { Reduced } \\
\text { Tension } \\
\text { (004 option) }\end{array}$} & $\begin{array}{c}\text { Wire Rope } \\
\text { Reduced } \\
\text { Acceleration } \\
\text { (004 option) } \\
\text { (G's) }\end{array}$ \\
\hline 2 & 2 & 50 & 34 & 9.5 & $>50$ & 16 & 4.4 & \begin{tabular}{|l|}
28 \\
\end{tabular} & - & - & 16 & 4.4 & 14 \\
\hline 3 & 3 & 75 & 24 & 6.7 & $>50$ & 14 & 3.9 & 16 & - & - & 14 & 3.9 & 15 \\
\hline 4 & 4 & 100 & 24 & 6.7 & $>50$ & 11 & 3.1 & 12 & - & - & 11 & 3.1 & 15 \\
\hline 5 & & 125 & 34 & 9.5 & $>50$ & 8 & 2.2 & 7 & - & - & 8 & 2.2 & 6 \\
\hline 10 & 10 & 250 & 34 & 9.5 & $>50$ & 16 & 4.4 & 28 & $16 \quad 4.4$ & 19 & 16 & 4.4 & 14 \\
\hline 15 & & 390 & 24 & 6.7 & $>50$ & 14 & 3.9 & 16 & - & - & 14 & 3.9 & 15 \\
\hline 20 & 20 & 500 & 24 & 6.7 & $>50$ & 11 & 3.1 & 12 & - & - & 11 & 3.1 & 14 \\
\hline 25 & 25 & 640 & 34 & 9.5 & $>50$ & 8 & 2.2 & 7 & $\begin{array}{ll}8 & 2.2 \\
\end{array}$ & 7 & 8 & 2.2 & 6 \\
\hline 30 & 30 & 750 & 24 & 6.7 & $>50$ & 14 & 3.9 & 16 & - & - & 14 & 3.9 & 15 \\
\hline 40 & & 1000 & 24 & 6.7 & $>50$ & 11 & 3.1 & 12 & - & - & 11 & 3.1 & 12 \\
\hline 50 & 50 & 1250 & 34 & 9.5 & $>50$ & 8 & 2.2 & 7 & 2.2 & 7 & 8 & 2.2 & 5 \\
\hline 60 & & 1500 & 24 & 6.7 & 27 & 7 & 1.8 & 2 & 1.8 & 5 & 7 & 1.8 & 6 \\
\hline 80 & 80 & 2000 & 19 & 5.3 & 16 & 5 & 1.4 & 2 & 1.4 & 2 & 5 & 1.4 & 3 \\
\hline
\end{tabular}

DIMENSIONAL INFORMATION

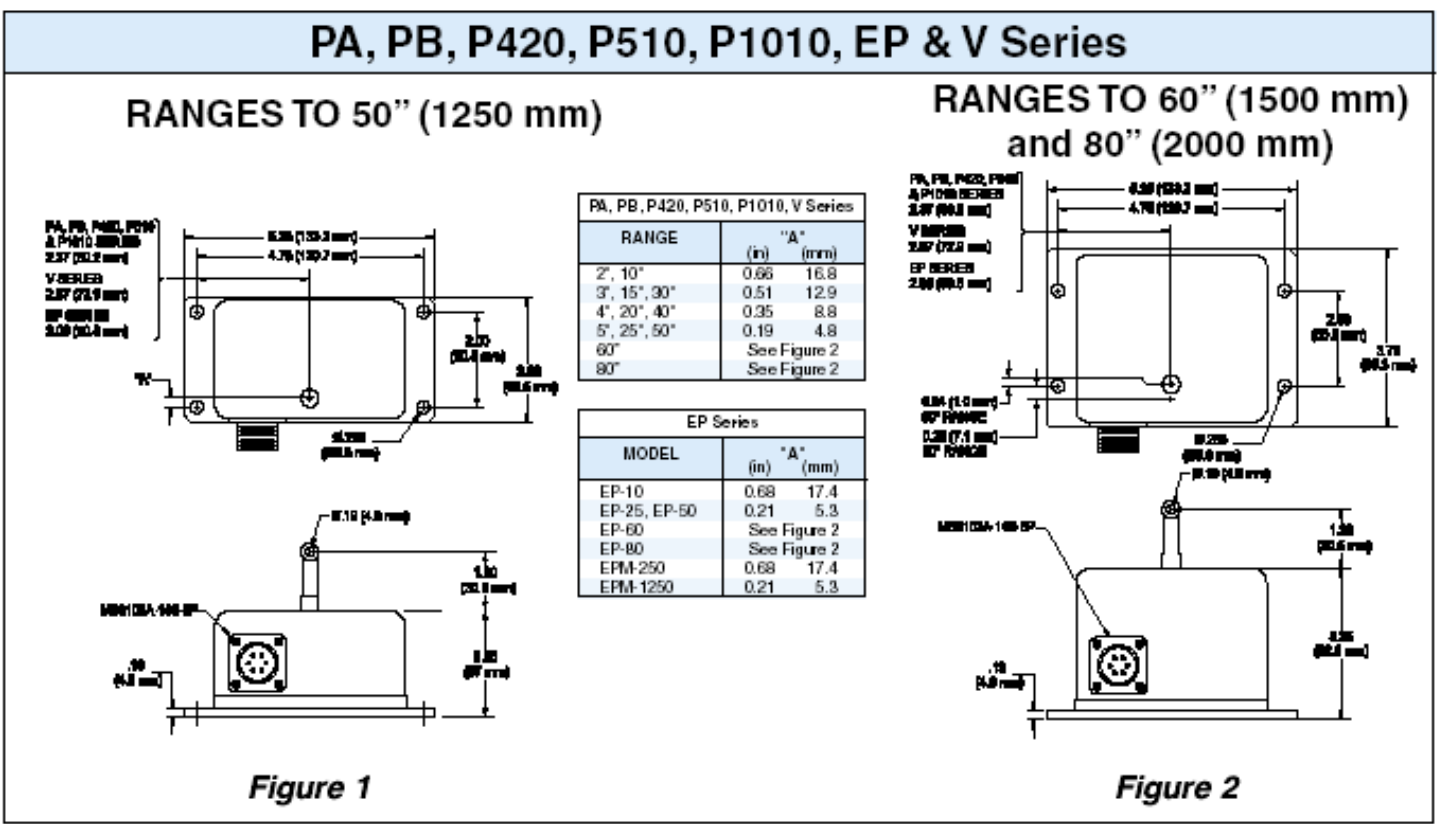

TF. Uni 4175 SW Research Way, Corvallis, OR 97333 | Tel: 541-757-3158 | Fax: 541-757-0858| Email: sales@ unimeasure.com 


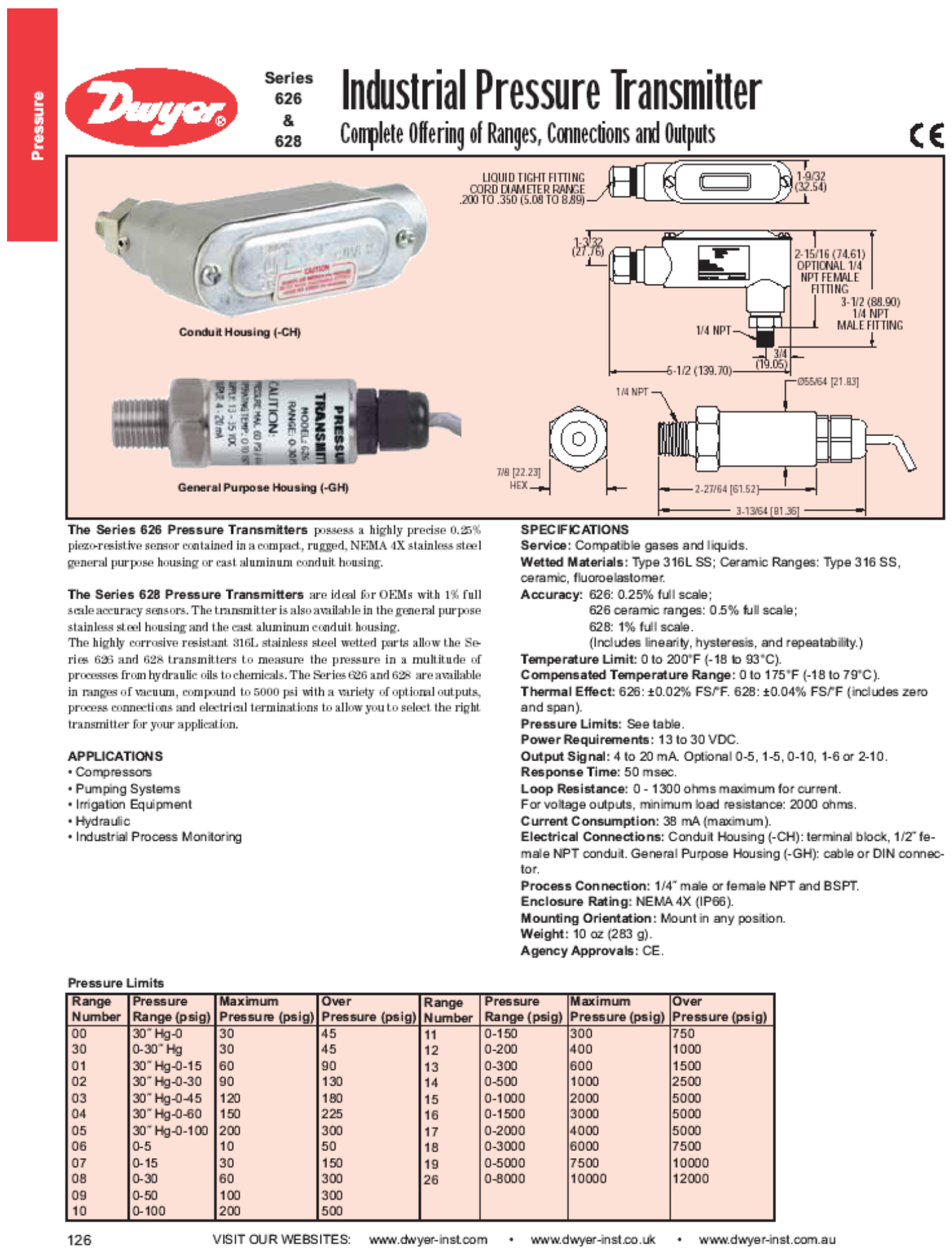


Ordering Chart

\begin{tabular}{|c|c|c|c|c|c|c|c|c|}
\hline Accuracy & 626 & & & & & & & $\begin{array}{l}0.25 \% \text { Full Scale Accuracy } \\
1.0 \% \text { Full Scale Accuracy }\end{array}$ \\
\hline Range & & $\begin{array}{l}-30 \\
-00 \\
-01 \\
-02 \\
-03 \\
-04 \\
-05 \\
-06 \\
-07 \\
-08 \\
-09 \\
-10 \\
-11 \\
-12 \\
-13 \\
-14 \\
-15 \\
-16 \\
-17 \\
-18 \\
-19 \\
-26 \\
\end{array}$ & & & & & & $\begin{array}{l}0-30^{\circ} \mathrm{Hg} \text { Vacuum } \\
30^{-1} \mathrm{Hg} \text { Vacuum }-0 \mathrm{psi}^{*} \\
30-0-15 \mathrm{psi}^{*} \\
30-0-30 \mathrm{psi}^{*} \\
30-0-45 \mathrm{psi}^{*} \\
30-0-60 \mathrm{psi}^{*} \\
30-0-100 \mathrm{psi}^{*} \\
0-5 \mathrm{psi} \\
0-15 \mathrm{psi} \\
0-30 \mathrm{psi} \\
0-50 \mathrm{psi} \\
0-100 \mathrm{psi} \\
0-150 \mathrm{psi} \\
0-200 \mathrm{psi} \\
0-300 \mathrm{psi} \\
0-500 \mathrm{psi} \\
0-1000 \mathrm{psi} \\
0-1500 \mathrm{psi} \\
0-2000 \mathrm{psi} \\
0-3000 \mathrm{psi} \\
0-5000 \mathrm{psi} \\
0-8000 \mathrm{psi}\end{array}$ \\
\hline Housing & & & $\begin{array}{l}-\mathrm{CH} \\
-\mathrm{GH} \\
\end{array}$ & & & & & $\begin{array}{l}\text { Conduit Housing } \\
\text { General Purpose Housing }\end{array}$ \\
\hline $\begin{array}{l}\text { Process Con- } \\
\text { nection }\end{array}$ & & & & $\begin{array}{l}-P 1 \\
-P 2 \\
-P 3 \\
-P 4 \\
-P 5 \\
-P 8 \\
-P 9 \\
\end{array}$ & & & & $\begin{array}{l}1 / 4^{\prime \prime} \text { male NPT } \\
1 / 4^{\prime \prime} \text { female NPT } \\
1 / 4^{\prime \prime} \text { male BSPT } \\
1 / 4^{\prime \prime} \text { female BSPT } \\
1 / 4^{\prime \prime} \text { female SAE with Refrigerant Valve Depressor } \\
1 / 8^{\prime \prime} \text { male NPT } \dagger \\
1 / 2^{\prime \prime} \text { male NPT } \dagger \\
\end{array}$ \\
\hline $\begin{array}{l}\text { Electrical Con- } \\
\text { nection }\end{array}$ & & & & & \begin{tabular}{l|}
$E 1$ \\
$-E 2$ \\
$-E 3$ \\
$-E 4$ \\
$-E 5$ \\
$-E 6$ \\
\end{tabular} & & & $\begin{array}{l}\text { Cable Gland with } 3 \text { of Prewired Cable } \\
\text { Cable Gland with } 6 \text { ' of Prewired Cable } \\
\text { Cable Gland with } 9^{\prime} \text { of Prewired Cable } \\
\text { DIN Connectort } \\
1 / 2^{\prime \prime} \text { female NPT Conduitł } \\
\text { M-12 } 4 \text { Pin Connector }\end{array}$ \\
\hline Signal Output & & & & & & $\begin{array}{l}-\mathrm{S} 1 \\
-\mathrm{S} 2 \\
-\mathrm{S} 3 \\
-\mathrm{S} 4 \\
-\mathrm{S} 5 \\
-\mathrm{S} 6 \\
\end{array}$ & & $\begin{array}{l}4-20 \text { mA } \\
1-5 \text { Volt } \\
2-10 \text { Volt } \\
0-5 \text { Volt } \\
0-10 \text { Volt } \\
1-6 \text { Volt } \\
\end{array}$ \\
\hline Options & & & & & & & \begin{tabular}{|l|}
-AT \\
-NIST \\
-LED
\end{tabular} & $\begin{array}{l}\text { Aluminum Tag } \\
\text { NIST Traceable Certificate } \\
\text { Bright Red LED displaył }\end{array}$ \\
\hline
\end{tabular}

† Avalable with -GH Housing only

$\mp$ Available with $-\mathrm{CH}$ Housing only

"Optional ceramic sensor available. Add "C" to the range (Ex. 626-00C-GH-P1-E1-S1).

Note: Ceramic transmitters are only available with 4-20 mA output and are not CE approved.

626 with LED Display (CH housing only)

Note: LED option is not NEMA $4 X$ rated.
Optional -E4 DIN Connector

(GH housing only)
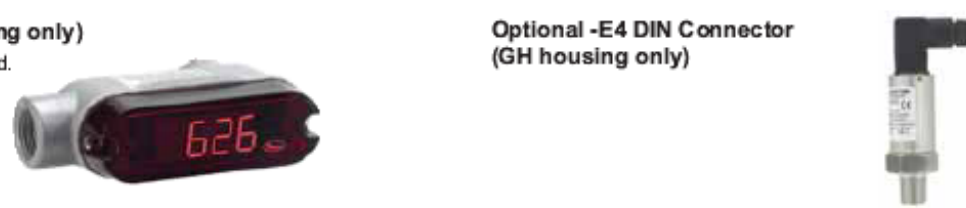